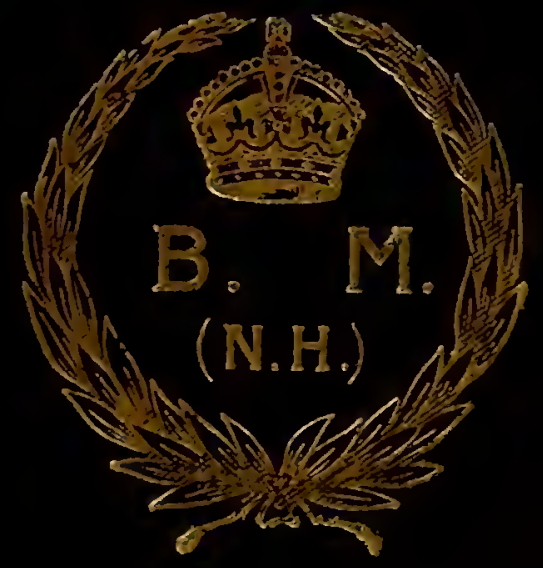




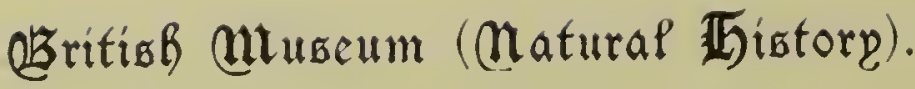

$$
\text { This is No. 2 of } 25 \text { copics of }
$$

"A Monograph of the Genus Sabicca," printed on Special papor. 

A MONOGRAPH OF THE GENUS S A B I C E A 


$$
\begin{aligned}
& B A L \\
& B E
\end{aligned}
$$




\title{
A MONOGRAPH OF
}

\author{
THE GENUS
}

\section{SABICEA}

\author{
BY \\ HERBERT FULLER WERNHAM, \\ B.Sc., F.L.S. \\ Assistant in the Department of Botany
}

\section{WITH TWELVE PLATES AND TEXT-FIGURES}

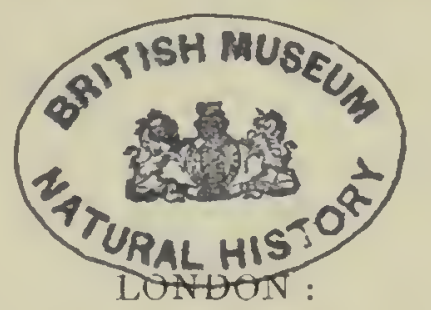

PRINTED BY ORDER OF THE TRUSTEES OF THE BRITISH MUSEUM

$$
A N D \text { SOLD BY }
$$

Longmans \& Co., 39, Paternoster Row, E.C.; B. Quaritch, it, Grafton Street, New Bond Street, W. ; Dulau \& Co., Ltd., 37, Sollo Square, W. ; and at the

British Museum (Naturai. History), Cromwell Road, S.W.

$$
1914
$$

(All rights reserved) 



\section{PREF A C E}

Mr. Wernham has devoted some time during the past few years to a revision of the material of the family Rubiaceæ contained in the Herbarium of the Department of Botany, and the Monograph of the genus Sabicea is the first of a series of monographs which it is hoped to publish as the result of his study of this family. In the course of his work Mr. Wernham has also studied the collections in the Herbarium of the Royal Gardens, Kew, and in the principal continental Herbaria, and cordial thanks are due to the Directors of these Institutions for the loan of plants and facilities for study.

A. B. RENDLE.

Department OF Botany,

March 11, 1914. 


\section{CONTENTS}

INTRODUCTION

\section{LIST OF PLATES}

Plate I. 1, S. umbrosa Wernham; 2-5, S. orientalis Wernham.

Plate II. S. laxa Wernham.

Plate III. 1, S. Pearcei Wernham; 2, 3, S. subinvolucrata Wernham.

Plate IV. 1-4, S. Moorei Wernham; 5-9, S. camporum Sprague.

Plate V. 1,2, S. mexicana Wernham; 3, 4, S. amazonensis Wornham.

Plate VI. 1-3, S. medusula K. Schum.; 4-6, S. parva Wernham.

Plate VII. 1-3, S. acuminata Baker ; 4-7, S. Batesii Wernham.

PlatF VIII. 1-4, S. gracilis Wernham; 5-8, S. mattogrossensis Wernham.

Plate IX. 1-3, S. lanuginosa Wernham; 4, 5, S. Dewerrei De Wild. var. glabra Wernham.

Prate X. 1, 2, S. Robbii Wernham; 3-5, S. floribunda K. Schum. var. paucinervis Wernham.

Plate XI. 1-4, S. xanthotricha Wernham; 5-7, S. Barteri Wernham.

Plate XII. Single flowers of species numbered according to the text. 



\section{INTRODUCTION}

History.-Sabicea dates from Aublet's "Plantae Guianenses," published in 1775. Here two species are described--S. cinerea and $S$. aspera; both are scrambling plants, as are the majority of the species which have been discovered since.

The generic name was devised by Aublet froin the common name for $S$. aspera used by the Galibis of Guiana-Sabi-Sabi. Presumably deeming it "barbaric," Schreber, in his "Genera Plantarum," 1789, replaces Aublet's name by Schwenlefelda-a name which the present rules for nomenclature happily compel us to discard.

Swartz added a Jamaican species in 1788 , S. hirta, and Ruiz and Pavon a Peruvian species, S. umbellata, in the following year. In 1805 Persoon included a Madagascar plant, S. diversifolia, hesitatingly but correctly in the same genus.

In 1818 Humboldt, Bonpland and Kunth published in their "Nova Genera et Species Plantarum, etc.," a fifth species of the genus, S. hirsuta. A chronological list of all the species appears on pages 2, 3; and it will be seen that between 1818 and 1849 , when our knowledge of West African plants was so substantially increased by the publication of Hooker's Niger Flora, only four more species, all American, were added.

The Niger Flora contains descriptions of the first five species recorded for the African continent. Not until nearly thirty years later was any addition made to the list, when, in 1877 , four more African species appeared in the Flora of Tropical Africa, under the authorship of Hiern.

Between that year and the end of 1912 twenty-one additional species have been described (see list), of which sixteen are African, four American, and one is from Madagascar. In the course of investigating the Rubiacer of Mr. and Mrs. Talbot's Nigerian collection last year, I was surprised to find so many as four new species of Sabicea, and thus I was led to a complete examination of the genus. This has revealed the existence of more than sixty 
additional species, all of which are described here, bringing the total number up to one hundred and six.

For the purposes of the present investigation I have examined all the relative material in the National Herbarium, the herbaria at Kew, and in Cambridge University; the Berlin herbarium, and the collection of Krug and Urban; the herbaria of the Museums at Paris and Madrid; the State herbarium at Brussels; the herbaria of Boissier, De Candolle, and Delessert, at Genera; and the Stockholm herbarium.

I take this opportunity to express my grateful obligations to the following gentlemen for the assistance which they have given me, and the facilities they have provided for my investigation of the several eollections under their control:-Dr. Stapf, and the staff of the Kew herbarium; Dr. Mloss, curator of the Cambridge Unirersity herbarium; Professor Lecomte and his staff at Paris ; at Geneva, M. Barbey and his eurator M. Beauverd, of the Boissier herbarium, Dr. Briquet, of the Delessert herbarium, and MM. De Candolle; Dr. De Wildeman, at Brussels; and Dr. Prosper Reyes, of the Universidad Central, Madrid. My sineere thanks are due also to Prof. Engler and Dr. Urban for placing at my disposal for a considerable period the valuable material in the Berlin herbarium and the Krug and Urban collection; and to Dr. Carl Lindman for rendering me a similar service in regard to the Stockholm herbarium. I have to record further my great indebtedness to Dr. Rendle, of the National Herbarium, who has been ready throughout with invaluable advice; and to the Trustees of the British Museum, for a substantial grant toward the expense of visiting the various Continental herbaria.

\section{SPECIES IN CHRONOLOGICAL ORDER.}

1775 aspera Aubl. cincrea Aubl.

1788 hirta Sw.

1789 umbellata (Schwenkfelda) R. \& P.

1805 diversifolia Pors.

1818 hirsuta IF. B. \& K.

1829 grisca Ch. \& Schl.
1840 cana Hook.

1841 glabrescens Benth. volutina Benth.

1849 calycina Beuth. capitcllata Benth. ferruginea Bonth. vonosa Bentl. Vogelii Benth. 
1877 cauliflora Hiern geantha Hiern pilosa Hiern segregata Hiern

1889 novogranatensis K. Schum.

1890 acuminata Baker Schumanniana Bïttner

1892 ingrata K. Schum.

1993 humilis S. Moore

1896 cuneata Rusby speciosa K. Schum.

1897 Dinklagei K. Schum. floribunda K. Schum.

1899 arborea K. Schum. trigemina K. Schum.
1901 Dewevrei De Wild.

1903 bicarpellata K. Schum. gigantostipula K. Schum. Gilletii De Wild. speciosissima K. Schum.

1901 camporum Sprague

1905 discolor Stapf

1906 Laurentii De Wild. longepetiolata De Wild.

1912 tchapensis Krause

1913 geophiloides Wernham pedicellata Wernham Talbotii Wernham xanthotricha Wernham

NEW SPECIES.

amazonensis
angolensis
angustifolia Boivin MIS.
asperula
Barteri
Batesii
boliviensis
brachiata
bracteolata
brasiliensis
brevipes
brunnea
Burchellii
cameroonensis
colombiana
composita
costaricensis
cruciata
Dewildemaniana
dubia
Duparquetiana Baill.
$\quad$ MS.
entebbensis

entebbensis

erecta Rusby MIS.
flagenioides
fulva
gigantea
glomerata
gracilis
guianensis
Hierniana
Johnstonii K. Schum.
$\quad$ IS.
lanuginosa
laxa
Lindmaniana
mattogrossensis
medusula K. Schum.
$\quad$ MS.
mexicana
Mildbraedii
mollis K. Schum. MS.
mollissima Benth.MS.
Moorei

orientalis panamensis pannosa paraënsis parva parviflora K. Schum. MIS.

Pearcei pseudocapitellata

Robbii rufa

Schaeferi setiloba seua Smithii stipularioides subinvolucrata

Trailii

Trianae

umbrosa

Urbaniana verticillata

Distribution. - The home of Sabicea is in the tropics of Africa, including M.adagascar, and of the New World, the large majority ( 80 per cent.) of the species being scrambling shrubs. The habit of the various species will be dealt with presently. In only two cases does the genus appear outside the tropics, namely, the widely-distributed $S$. hirsuta, which occurs in extratropical south-eastern Brasil (Santa Catharina), and S. grisea, which is recorded once from Paraguay. 
There are three main areas of distribution: 1st, the African continent; 2nd, Madagascar: 3rd, America.

Details of the distribution of all the species are indicated in the accompanying Tables. For the present purpose the African eontinent has been divided into seven, and America into nine, sub-areas.

The region richest in species is Western Africa, and particularly the Cameroons, * where no less than 35 speeies, 24 of them endemie, have been found. In Senegambia, the most northerly point of its oceurrence in the Old World, the genus is represented by one speeies only, S. venosa; the same speeies occurs fairly generally over West Afriea, as far south as the Quango, a tributary of the Congo. In Angola $S$. venosa is replaced by the allied, but quite distinct, $S$. angolensis; here it is aeeompanied by only one other speeies, the aberrant $S$. parviflora. $S$. calycina, a very well-marked species, has a distribution similar to that of $S$. venosa, with the exception that the Gold Coast is its most northerly and westerly station.

Eastern Tropieal Afriea is almost as poor in species as Angoli, only three having been found in this region: S. arborea, a small tree, and $S$. cntcbbensis and $S$. oricntalis, both related to S. venosa.

S. discolor and $S$. Vogelii oceur from Sierra Leone to French Guinea ; S. speciosa from Togoland to the Cameroons; S. gigantostipula, S. geoplitoides, S. Roblii, and S. floribunda in Nigeria and the Cameroons. S. capitellata, S. Laurentii, S. Dinllagei, and $S$. segregata have been found in the Congo basin as well as in the Cameroons. S. orientalis, apparently eommon in German East Africa, has been recorded onee as far west as the Mongala river, a tributary of the Congo.

All the other African species are endemic (see Table, p. 6) for the sub-areas named:-1 in Sierra Leone and Liberia, 8 in the Ivory Coast to Nigeria distriet, 24 in the Cameroons, and 10 in the Congo basin.

The 5 Madagasear speeies are all eonfined to that island (see, however, the comment on $S$. diversifolin under that species), and are peculiar in habit and strueture.

In the New World the northern and western limit of

* It must be remembered, howerer, that moro attention has been paid by collectors to this region than to the others, at least in reeent years. 
distribution is the Oaxaca district of Mexico, where the single species S. mexicana occurs. S. Tirsuta, which has the widest distribution of any of the species, extends from Guatemala in the north-west, eastward through Central America, along the north of South America to Cayenne; in the west, from Colombia southward to Peru ; a disconnected area appears in south-eastern Brasil from Bahia to Blumenau, which is south of the Tropic of Capricorn; and finally, this species occurs in the West Indian islands of Porto Rico and (as a variety) St. Thomas.

The rest of the American species are relatively limited in distribution. S. brasiliensis occurs fairly generally over eastern and southern Brasil, and in Bolivia. S. grisea extends from Ceara in the north over eastern Brasil to Rio de Janeiro in the south; it is recorded once also from Paraguay, where it is the only species. For the rest, 3 species are peculiar to Central America; $S$. hirta is the only species found in Jamaica, where it is endemic; this, $S$. hirsuta, and $S$. cinerca being the only West Indian species. Eleven species are peculiar to western South America, 3 to Guiana, Venezuela and Trinidad, 5 to the Amazon basin, 3 to Bolivia, 3 to Matto Grosso (Central and Southern Brasil), and 2 to Eastern Brasil.

In all, 62 species belong to the tropics of the African continent, 5 to Madagascar, and 39 to the New World.

Altitude.- In many cases the altitude of occurrence of the species is not given. From those cases in which we have information in this regard it would appear that the genus occurs at all levels from the sea-shore (S. Robbii) to 8000 feet ( $S$. cauliflora); and it is gathered that the species in many cases prefer a damp habitat-moist meadows, river-banks, etc. The following have not been found below 5000 feet: $S$. arborca, 5000-6000 feet; S. Schacfcri, 5,800-6,500 feet; S. cauliflora, $5000-8000$ feet.

HABIт.-The large majority of the species are shrubs; two are trees-S. arborea of Zanzibar and $S$. gigantca from the Congo basin. The following are small, prostrate, and more or less herbaceous: S. medusula, S. Mildbraedii, S. geophiloides, and S. Bartcri, all West African, and the Brasilian S. parva. The manifestly shrubby habit serves to distinguish Sabicea at sight from Coccocypselum, an American genus of herbaceous species. 


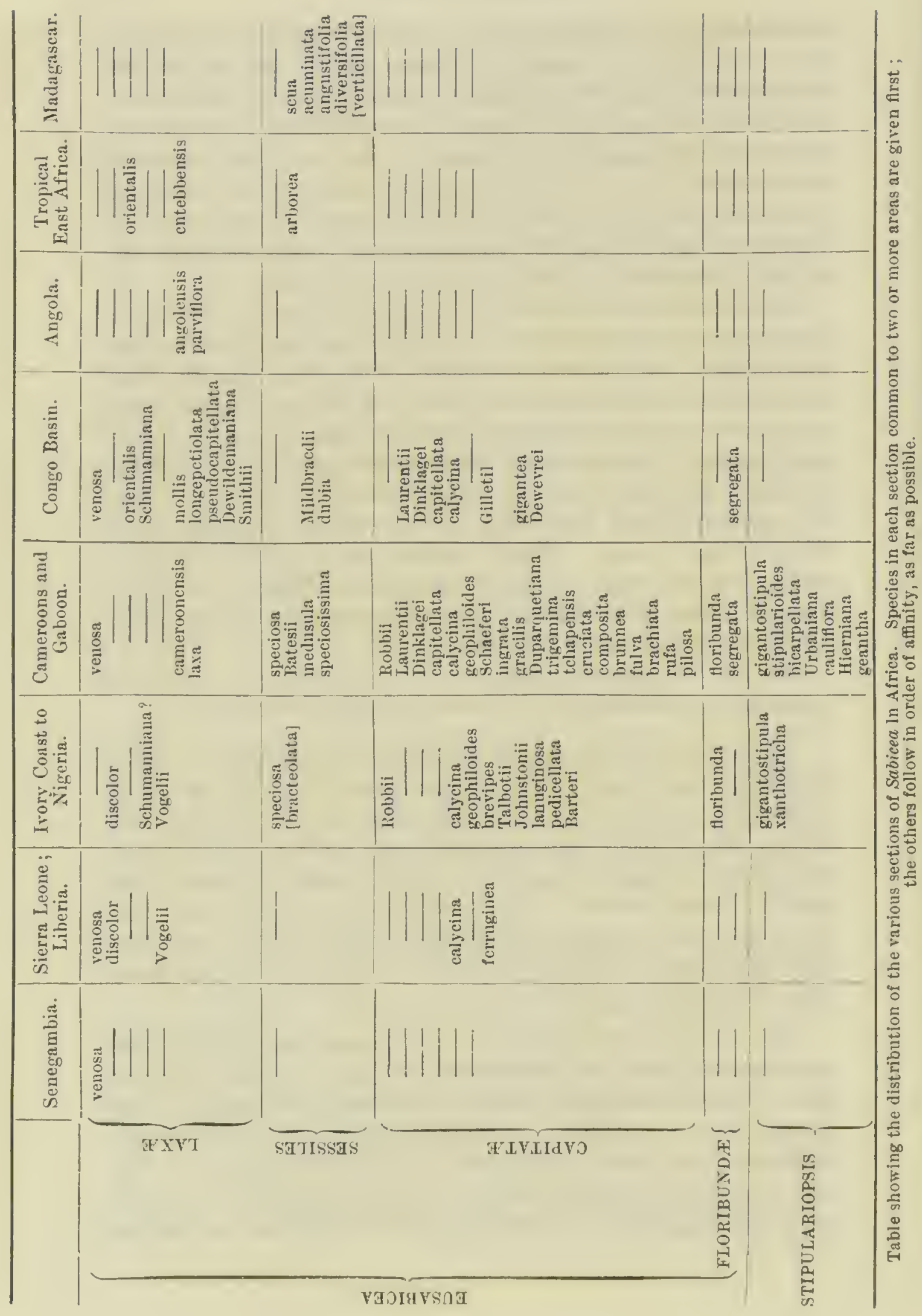




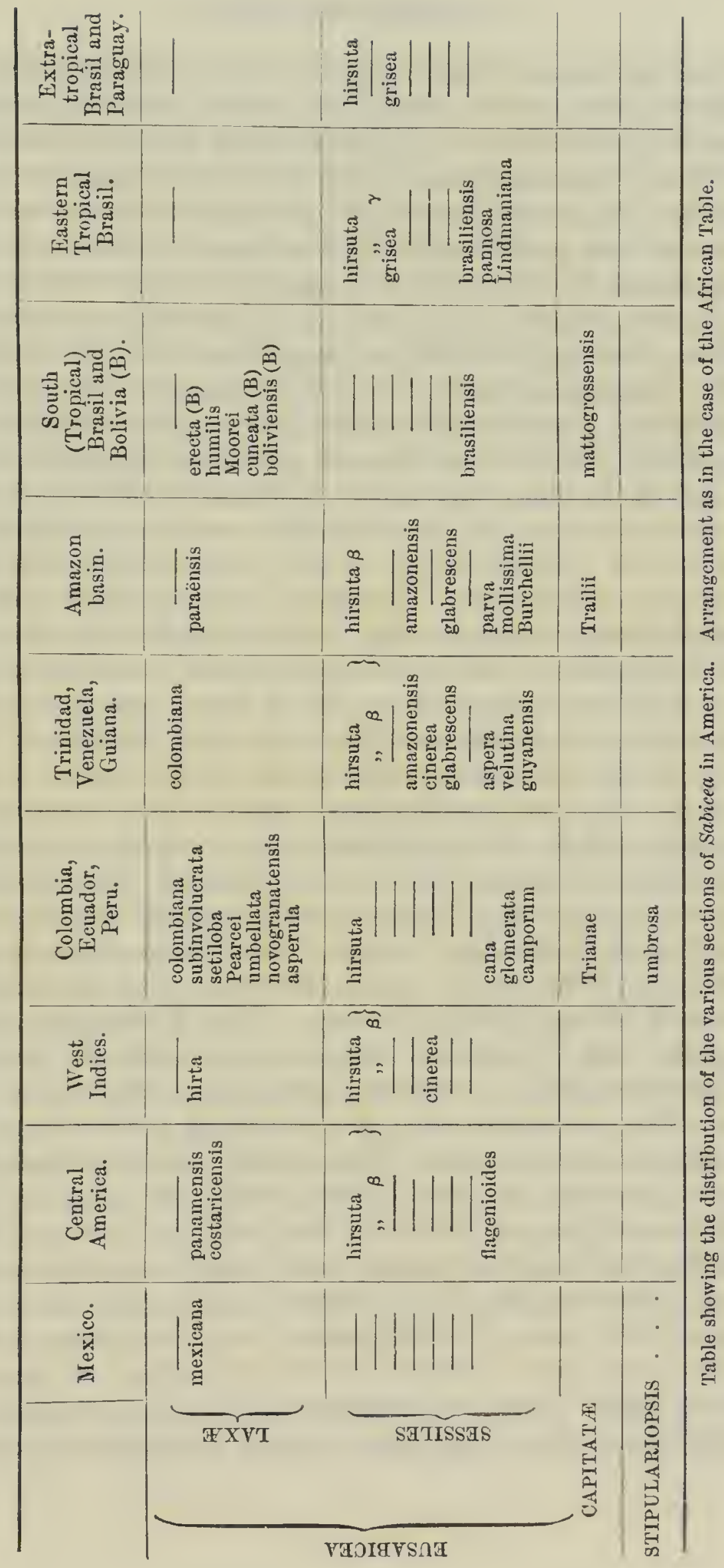


Most of the species of Sabicea, moreover, are climbers of the most primitive type-namely, scrambling plants, reaching a height of 10 to 15 feet, more or less, unprovided with hooks, tendrils, or other climbing devices. They climb over hedges or scrub bordering the tropical forest, often along river-banks; we have seen that they prefer moist situations for the most part. The exceptions to the seandent habit, apart from the herbs just now mentioned, number barely a score in all ; but they are of especial interest, for they indicate an important trend of evolution within the genus-namely, a tendency from the climbing to the erect habit. That this, and not the reverse, has been the course of evolution seems to be evidenced, e.g. by the migration of the genus from the long established conditions of the tropical scrub to open situations in the derivative campos or savannahs, where it is represented by a few erect forms; and these latter are mostly of a specialised habit. They may be either bushes of moderate size-such as the Bolivian $S$. erecta and the Central American S. panamensis; or they may be quite small and subherbaceous, like $S$. brasiliensis, which grows in the plains of Brasil and Bolivia up to an altitude of 5000 feet; or $S$. camporum, barely 2 feet high, which occupies a similar place in the Colombian plains. Two more species, S. Inmilis and $S$. Moorei of Matto Grosso, complete the list of American erect forms, with one exception, $S$. umbrosa-to be referred to presently; these are lowly, erect and subherbaceous, a foot or 18 inches high at most. In all these cases the erect species are more or less nearly related to typically scandent species of the neighbouring thickets. Thus $S$. erecta has obvious affinities with $S$. setiloba; $S$. panamensis witl $S$. paraënsis; S. brasiliensis and $S$. camporum with $S$. cana, S. guianensis and their allies; $S$. humilis and $S$. Moorei with $S$. umbellata, etc.

The case is somewhat different with the five Madagascar species; for they are mostly erect bushes with no very clear affinities with any of the rest. The habit of $S$. angustifolia is significant in its subsarmentose nature, and it may represent the nearest surviving ally of an ancestral climbing race ; but in other respects this species is closely allied with $S$. acuminata, $S$. diversifolia, and S. scua. We shall discuss the affinity of these four species later. They are peculiar in their characters of anisoplyylly (and this is least pronounced in the relatively primitive 
$S$. angustifolia), and of fimbriated or laciniate stipules-features which are found combined in only two other species, S. Mildbrcedii and $S$. dubia, both subherbaceous forms from the Congo region. Anisophylly occurs in two other species $(q . v$.$) , both from$ the Cameroons, S. medusula, a creeping herb, and S. Batesii. In the latter the inequality of the leaves is so extreme as to result in the complete suppression of one leaf of each nodal pair, so producing a pseudo-alternate arrangement. The same condition appears in some of the ultimate twigs in S. diversifolia.

The fifth Madagascar species, S. verticillata, differ's from all the others of the genus in having a whorled leaf-arrangementthree subequal leaves arising at each node; and the aberrancy of this species is further marked by the solitariness of the flowers. It resembles the other Madagascar species, however, in having laciniate stipules.

The remaining exceptions to the climbing habit are the nine species which I have grouped as a subgenus Stipulariopsis. These are more or less lank erect suffiutescent forms with large and relatively few leaves, and ample stipules which conceal or tend to conceal the dense axillary exinvolucrate clusters of flowers. All are West African, with the exception of the Colombian S. umbrosa. The megaphylly and general habit is suggestive of the origin of this subgenus through a geophilous tendency; and indeed this is foreshadowed in forms like $S$. humitis and $S$. Moorei, which display, especially in the latter case, a tendency to the formation of few and large leaves; while the low-growing erect shoots, arising from a stout rootstock, point in the same direction, namely, to the geophilous habit. S. xanthotricha, however, is, according to Mr. Talbot, its discoverer, a rather large bushy shrub, and should perhaps not be classed in this geophilous subgenus. In this case $S$. xanthotricha and $S$. Batesii would constitute the two sole exceptions to my primary two-fold division of the genus, based, as will be seen from the key, on the size of the leaves. In the case of S. Batesii the megaphylly is not improbably correlated with the extreme anisophylly - a compensatory tendency to maintain the effective assimilating area (see Pl. VII, 4).

Critical FloRal ChaRACTERS.-The critical features of the genus are displayed in the floral characters. The valvate 
restivation of the corolla, coupled with the multiovular ovary maturing into a many-seeded berry, leads indubitably to the inclusion of Sabicea in the tribe Mussander. The generie eharacters are as follows. In the first place the inflorescences are axillary, in contrast with the terminal inflorescences in one or two of the better known genera of the tribe, such as Mussixnda and Isertiu. Secondly, the inflorescence, though frequently associated with a very definite involuere of free or sub-free bracts, is never enclosed in a campanulate involucre (see Pls. VIII, IX, X). This is the principal eritical distinction between Sabicea and the allied African genus Stipulariu. The latter comprises two or three species of shrubs, with silky or felted indumentum, and large elliptical or obovate leaves with conspicuous broad foliaceous stipules. Stipuluria is a near generic ally of Sabicea, and we shall need to revert to it later.

For the rest, the calyx of the 4-5-merous hermaphrodite flowers has in most cases elongate or conspicuously large lobes. As in other Mussender, and indeed in many other Rubiacex generally, the lobes in the individual flower are frequently unequal and of differing shapes-a tendency which reaches a climax in the familiar petaloid calyx-lobe of many species of Musscude; * but in Sabicen the inequality is in no case very pronounced. The calyx-limb is divided with few exceptions below the middle, often almost to the base, usually into linear, sometimes lanceolate or subulate, occasionally ovate or elliptical lobes. The corolla-linb, on the other hand, has typically a relatively long narrow tube, but little widened above, with proportionately short lobes.

This relative elongation of the calyx-lobes, and of the slender corolla-tube distinguishes Sabicea from the allied Uroplyyllum, in which the calyx-limb is shortly, sometimes minutely, toothed, and the corolla-tube is relatively short and broad. The flower's in the latter genus are moreover noticeably small. In Sabicea the total length of the corolla is very rarely less than 6 or $7 \mathrm{~mm}$. (S. hirsuta, $S$. asperula)-and this is above the maximum for Urophyllum; the most usual length is from $1.5 \mathrm{~cm}$. to $2 \mathrm{~cm}$. The latter is excceded in S. Schumanniuna $(1.8$ to $2.5 \mathrm{~cm}$.), $S$. amazonensis $(2.5 \mathrm{~cm}$.$) , S$. pilosa $(2.5 \mathrm{~cm}$. to $3.5 \mathrm{~cm}$.$) ,$ S. speciosa (3-4 cm.), S. umbrosa (upwards of $4 \mathrm{~cm}$.), and finally

* See Joum. Bot. li. (1913), 233. 
in S. speciosisima, with flowers $10 \mathrm{~cm}$. long. The smallest flowers occur in S. Mildbraedii, with corolla only $4 \mathrm{~mm}$. long, S. camporum, 5-6 $\mathrm{mm}$, and in the section Floribundx-two species in which the corolla does not exceed $5 \mathrm{~mm}$. in length. The flowers of most of the representative species are figured in Plate XII.

The gynæcium is in the large majority of the species isomerous with the corolla, or, at any rate, the carpels number more than two; the ovary is usually 4-5-locular, as in Urophyllum.* The ovary is, however, bilocular in most of the species of Stipulariopsis, and in two or three species of Eusabicea, e.g. S. arborea, S. segregata; but the total number of species with only two loculi in the ovary does not exceed about half a score. This is in contrast with Stipularic, in which the ovary is frequently two-chambered-a curious, if not significant, point of similarity to the large-leaved subgenus of Sabicea mentioned above (v. also infra, p. 17). The ovary matures to a small round 4-5-locular berry containing several seeds; in Stipularia the fruit is much larger and ellipsoidal.

\section{CLASSIFICATION OF THE SPECIES.}

Having enumerated the constant and critical characters which determine the genus, we have now to deal with the variable features, and to attempt to discover if possible the lines along which evolution has operated within the genus.

The principal characters which vary with the species, but which are constant for each individual species, are 1st, the indumentum, especially of the leaves; $2 \mathrm{nd}$, the nature of the inflorescence, whether relatively lax or dense and compact, whether sessile or pedunculate, involucrate or ex-involucrate; $3 r d$, the actual length of the calyx-lobes $†$ and their shape; and 4th, the shape and relative size of the corolla-tube and lobes.

Indumentum.-The leaf-indumentum is remarkably constant for each species, particularly in regard to the presence or absence

* A polycarpellary ovary-or, at any rate, an ovary composed of more than two carpels-is the excoption in Rubiacer, being found in about onetenth, only, of the total number of species composing the family (see New Phytologist xi. 223). In the remaining nine-tenths the ovary is bicarpellary, and, with very rare exceptions, bilocular.

$\dagger$ In cases where the calyx-lobes are appreciably unequal, the largest is referred to invariably in what follows, throughout. 
of felt or arachnoid covering on the lower surface. In fact, $S$. venosa $\beta$. anomala is the only exception to this constancy, which is thus of considerable value in the practical determination of the species. In a few cases the arachnoid covering is deciduous ol' scanty, the pilose part of the indumentum predominating; such may be observed in specimens of $S$. mollis, $S$. orientalis, and $S$ rufa. None of the species are quite glabrous, except, perhaps, $S$. geantha; nor do many even approach the glabrous condition; Sabicea is essentially a hairy genus.

INFLORESCEXCE. - We have already hinted at a primary division of the genus, based upon the vegetative habit; the further grouping of the species is here made to depend upon the inflorescence. The latter is of three distinct types, namely, Ist, more or less lax at maturity, the inflorescence-branches being apparent; 2nd, closely compacted, both flowers and inflorescence sessile or subsessile; and $3 \mathrm{rd}$, a compact involucrate pedunculate head.

It will, perhaps, be readily conceded that the last-named type is relatively the most adranced, the looser and less definite type being by contrast the most primitive; just as the capitulum of Compositie is more advanced than the umbel of Umbellifere or than any diffuse inflorescence.

We will for the moment set aside the subgenus Stipulariopsis, with its 9 large-leaved, erect, geophilous species. I have divided the remaining 97 species, which constitute the subgenus Eusabicea, into four sections, in accordance with the inflorescence. In the first two sections, including 63 species, the bracts are usually inconspiouous, and they do not form a definite involucre. There are one or two apparent exceptions (S. cinerea, S. amazonensis, $\mathrm{Pl} . \mathrm{V}, 3$, see key) in which the bracts are often manifest and subinvolucrate; but the inflorescence is never a compact and definite involucrate stalked head as in the third section, Capitati.

The first section (Lax:) includes those species-31 in allin which the inflorescence is more or less lax at maturity (Fig. 1). In the 32 species of the second section, ${ }^{*}$ Sessiles, the branches of the inflorescence, the peduncle and pedicels are all suppressed or almost so, the flowers being disposed in dense axillary clusterssave in those very rare cases in which the flowers are solitary or

\footnotetext{
* The doubtful S. verticillata and $S$. bractcolata are included.
} 
subsolitary. The third section, Capitata, includes 32 species. In the fourth, Floribundx, the inflorescence is diffuse and compound, each partial inflorescence being associated with a

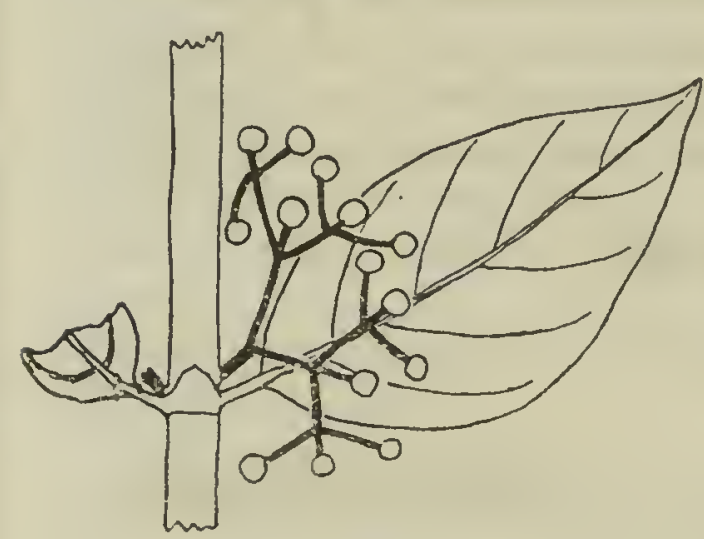

I. Laxis.

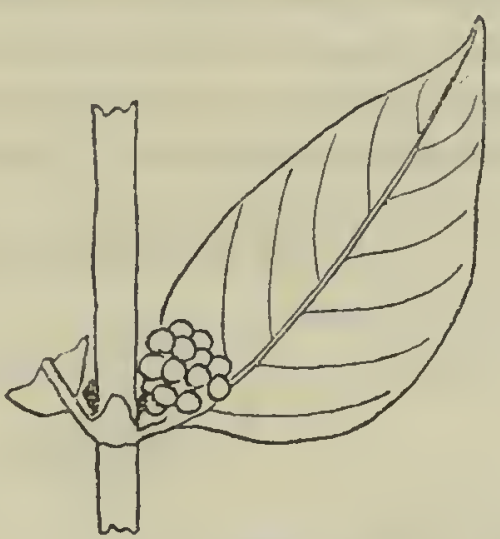

II. Sessiles.

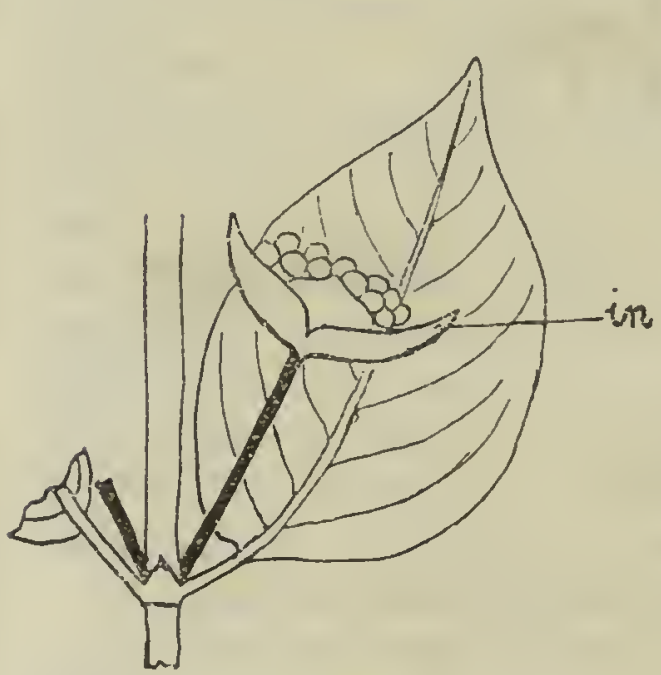

III. Capitate.

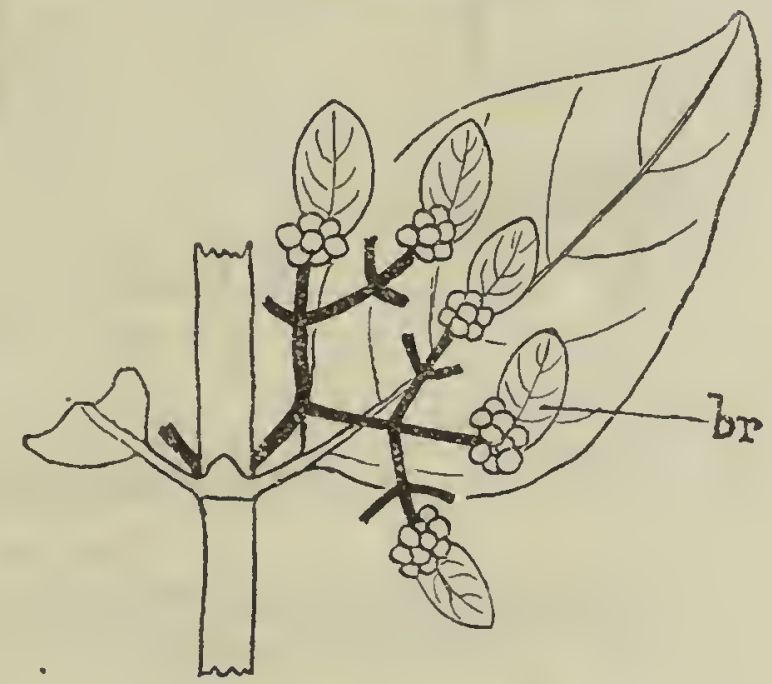

IV. FLORIBUND无.

FIG. 1.

Diagram showing types of inflorescence in each of the four sections of Eusabicea. in, involucre; $b r$, bract. Flowers represented by small circles.

more or less conspicuous foliaceous bract (see Fig. 1). Two species only-S. segregata and S. floribunda (see Pl. X, 3, 4) -are classed in this section.

The main course of evolution within the genus would appear to have been a progress from Laxi-as represented by S. venosa, $S$. paraënsis, etc.-chiefly along two diverging lines, one producing Sessiles, the other Capitate. On primit facie grounds it is not improbable that the lax preceded the compact type 
of inflorescence in the course of descent. The latter would arise by the suppression of the floral axes in response to the biological advantage of dense aggregation of the flowers; for by this means greater conspicuousness is secured for the purpose of attracting insect-visitors, which can, at the same time, pollinate a large number of flowers at a single brief visit. Such is the advantage of the head in Conpositæ - the most successful single

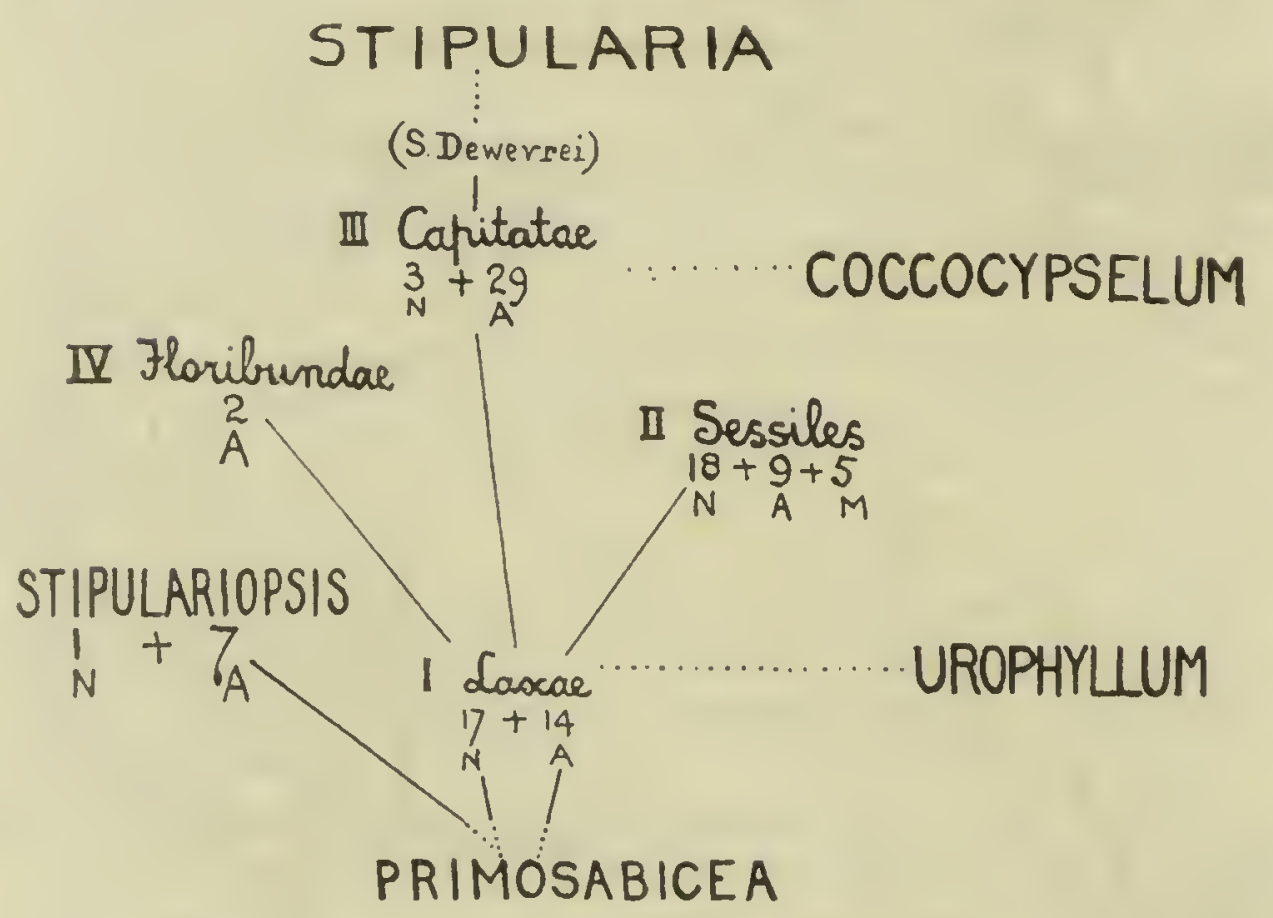

FIG. 2.*

Showing the origin and affinitios proposed for the various groups of Sabicea and their relation to allied genera.

group of flowering plants, if we may judge by the number of their species-the verticillaster of Labiatre, and so on.

Further, the suggestion that Sessiles and Capitate liave originated along separate lines emanating from an ancestral stock, represented by Laxæ, is supported by the circumstinces of the distribution of the species over the Old and New Worlds. The broad general affinities here proposed for the various groups of Sabicea are indicated in the accompanying diagram (Fig. 2), which indicates also the distribution. Thus Laxae are distributed fairly evenly between Africa (A) and America (N), for of the

* An African species of Stipulariopsis, S. stipularioidcs, has becn added sinco this diagram was completed; this makes 8 spocies for Africa instcad of 7 as shown. 
31 species included in this section, 17 belong to the latter and 14 to the former. Capitatie on the other hand are, with three exceptions, confined to the African continent; while of the 30 species of Sessiles, 18 are American against 8 African, the remaining 4 being the aberrant species from Madagascar (M). Moreover, the 8 African species are far from typical of Sessiles, for they include the curious S. medusula, S. Mildbraedii, S. Batesii, and $S$. dubia, with their exceptional habit, anisophylly, etc.; the tree $S$. arborea; and the large-flowered $S$. speciosa and $S$. speciosissima. The eighth, $S$. parviflora, has an inflorescence like $S$. aspera, with a tendency to laxity, and is barely evolved from the Laxie ancestry.

Broadly speaking, then, it may be supposed not unreasonably that the genus existed simultaneously in Africa and America as an ancestral race (PrimosabickA) with lax inflorescence, represented at the present day by e.g. S. venosa in Africa and S. paraënsis in America. In the latter area of distribution evolutionary development operated in the direction of producing dense, sessile axillary cluster's by the suppression of all the floral axes. In Africa, on the other hand, a special type of inflorescence was evolved-the stalked, involucrate capitulum. By the progressive development of the latter a new genus ultimately arose-Stipularia; this, it is significant to note, is confined to the African continent. S. Dewevrei and $S$. gigantea represent a transition to this genus in regard to the structure of the involucre (see descriptions of these species).

It is not improbable that the diffusely compound inflorescence of $S$. floribunda and $S$. segregati represents a third type of advance upon the primitive Laxæ; and in accordance with this idea, I have indicated this third section Floribundre as an off-shoot from the Laxæ line represented by $S$. venosa (Figs. 2,3). The three types of elaborated inflorescence, together with the primitive Laxie type, are shown diagrammatically in Fig. 1; and the four types are critical for the four sections of the subgenus Eusabicea respectively. The Floribundre type may be conceived as derived from the Laxie type by the substitution of a group of flowers for a single flower in the latter, and the addition of a more or less conspicuous bract to each group so formed (see Pl. X, 4).

A more detailed evolutionary tree is appended (Fig. 3), in which 
all the species of Eusabicea,* with their mutual affinities as proposed in this monograple, are indieated. The lower eircle includes Lax: within its circumference, and the upper, Capitate. Near the point of contaet between these two $S$. Vogelii lies; the inflorescence in this species varies from relative laxity to the compactness of a stalked head, with a more or less well-marked involucre. It may be regarded as transitional between the two sections. In the same way $S$. aspera possesses an inflorescenee of varying laxity, and is trausitional between Laxa and Sessiles.

The diagrain shows that the American Sessiles are conneeted upon one continuous shoot of the evolutionary tree whieh bears all the American Laxæ upon its lower branches. The three American Capitate- S. Trailii, S. mattogrossensis, and S. Trianae - have manifest afinities with Laxe as represented by $S$. umbellata, S. hirta, etc.

Of the subgenus Ensabicea there remain for consideration the Sessiles of Afriea (8 spp.) and Madagascar (4 spp.)-12 species in all. These are, as we have seen, more or less isolated in affinity; but their features of mutual resemblanee suggest the possibility of a common ancestry in the Primosabicean stockexeept, perhaps, in the case of the large-flowercd and imperfectlyknown S. speciosissima (Pl. XII, 40). S. diversifolia of Madagascar and $S$. arborea from the neighbouring district of Zanzibar, are similar in their erect habit, general facies and indumentum. We would suggest that $S$. arborea and $S$. speciosa may be the Sessilesdescendants of a Laxie-line represented by $S$. parviflora and $S$. Smithii, two African species on the border between Laxie and Sessiles (see Fig. 3). In a precisely similar way we have proposed that the African Capitate may be tho descendants of a Laxa-line represented by $S$. Vogelii, which is on the border between Laxie and Capitatie. In the latter case, however, we have been able to suggest the anecstry more remotely in Laxa, tracing it to typical species of that primitive section such as $S$. venose and $S$. discolor.

S. Batesii, S. Mildbraedii, S. dubia, and S. medusula-all Sessiles of the African continent-are linked by many points of resernblance, the most striking being anisophylly; they are refernble, in all probability, to a relatively recent common ancestry on a line emanating, presumably, from the $S$. arborea

\footnotetext{
* Except the doubtful S. bracteolata and S. verticillata.
} 


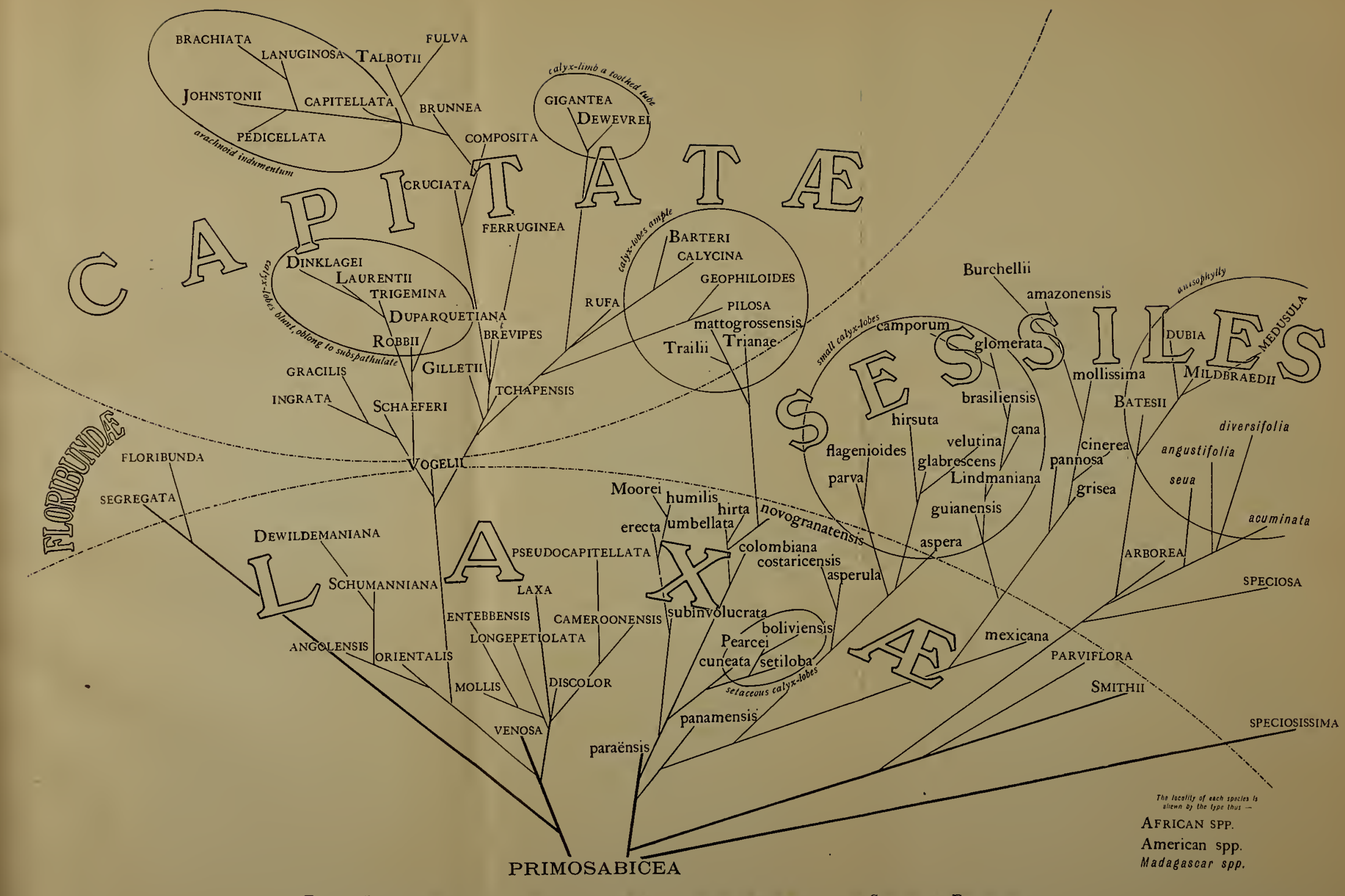

Fig. 3.-Diagram showing the ortgin aNd mutual afrinities proposed for the Species of Eusabicea. 

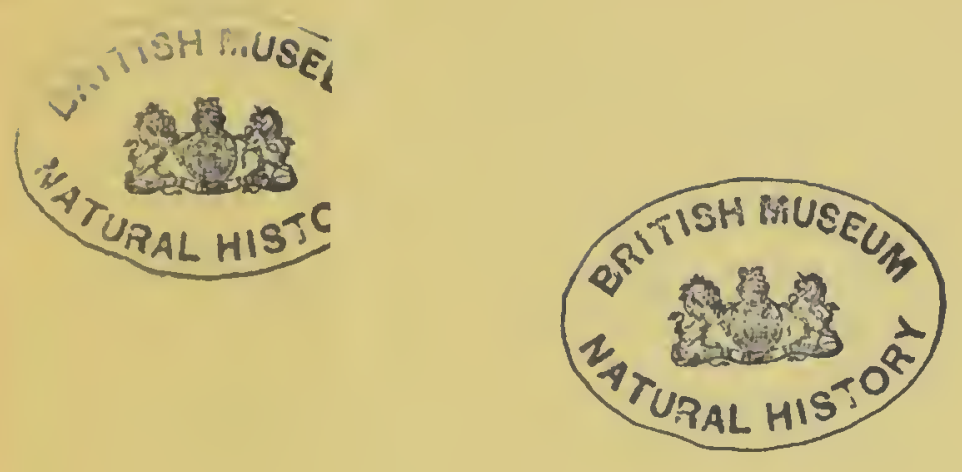
line just mentioned. The four Madagascar species (Fig. 3) may have arisen similarly. S. speciosissima, our knowledge of which is based upon a single indifferent specimen, may have originated independently as indicated in the diagram.

Turning now to the subgenus Stipulariopsis, it is suggested that it may have arisen by the operation of a tendency to geophily from forms resembling Eusabicea; and S. humilis and S. Moorei, we have seen, reflect the actual progress of such a. tendency. These two species are, however, American, and they have no manifest connection with S. umbrosa, the only American species included in Stipulariopsis. The non-climbing African species reveal no tendency to geophily; they are bushes, like $S$. diversifolia, trees, like $S$. arborea, or prostrate herbs, like S. medusula.

Nor is there manifest relationship between any African species of Eusabicea and any species of Stipulariopsis; and the details of the origin of the latter subgenus must remain in doubt for the present. Its inclusion in Sabicea is justified on the basis of the critical floral characters already enumerated; and it could be given separate generic rank only upon the inconstant and unsatisfactory ground of vegetative characters and the number of carpels in the ovary. The inflorescence in Stipulariopsis is usually of the Sessiles type, the only manifest exception being $S$. geantha, in which the inflorescence is lax. For the rest, it will be seen from the figures of the flowers that they are essentially Sabicean in appearance and external structure (see Pl. XII).

The CALYx has proved to be of considerable value in classifying the species. There is a clear distinction between species with calyx-lobes attaining $5 \mathrm{~mm}$. or more in length at an early stage, and accrescent in the fruit, and species in which the lobes are rarely more than about 3 or $4 \mathrm{~mm}$. even in the fruit. Thus, this distinction separates $S$. cana with short calyx-lobes from $S$. cinerea with long lobes; $S$. venosa from S. Schumanniana; and several others. This criterion is concerned only with the actual length of the lobes; relatively to the tube the calyx-lobes are nearly always long and narrow-this being one of the generic characters. Only in a few exceptional cases are the lobes relatively broad or shorter than the tube (see 
Pl. XII, 58, 97, 99, etc.). It will be seen from the figures that the calyx-lobes are generally linear-oblong to lanceolate, and often markedly acute-acuminate.

Many of what we may call the secondary, tertiary and higher branch-systems of our evolutionary tree are determined by the size and character of the calyx-lobes. Thus the whole subsequent branching (see Fig. 3) from the lines leading from Primosabicea respectively to Floribundæe, S. venosa, S. paraënsis, S. Smithii, etc., determined for the most part by the inflorescence, may be regarded as primary branch-systems; the lines in question are thickened in the diagram. The subsequent branching from the immediate tributaries of these lines will then be secondary branch-systems; similarly for the tertiary systems, and so on. To take examples, the species on the branching from the venosa-orientalis-line are characterised by long calyx-lobes. The Robbii-Dinllagei system exhibits a tendency to blunt, sub-spathulate lobes. In the giganteaDewerrei system the calyx-lobes are short and broad, and are appreciably shorter than the tube of the limb. In the pilosacalycina system the lobes tend to a broadly lanceolate or ovate contour, as do also those of the American novogranatensismattoyrossensis line. The calyx in the paraënsis-aspera system is short-lobed; in the paraënsis-mexicana-grisea system it is long-lobed.

The longer type of calyx-lobes is sometimes subulate (S. diversifolia, S. acuminata, S. angustifolia, S. speciosa, S. speciosissima; subulate-setaceous to setaceous in S. Urbaniana, S. xanthotricha, and $S$. ferruginea. There is evidence, too, of the evolutionary development of a calyx-limb with more or less setaceous lobes 4-5 $\mathrm{mm}$. long from a typically short-lobed calyx-i.e. in the progress from $S$. paraënsis to $S$. cuneata, S. setiloba, etc.

The more important divergences from the typical linear calyx-lobe have been mentioned; for the rest, the reader is referred to the Plates, in which figures of the flower in the typical cases appear.

Tire Corolda. - The range of variation is here less extensive, perhaps, than in the calyx. The most extreme differences are found in the actual size, as already noted (p. 10). There is a certain amount of variety, but not much, in the width of the 
corolla-tube and in the amount of widening in passing from the base to the mouth. The ratio between length of lobes and length of tube is never so much as $\frac{1}{2}$, the maximum being about $\frac{4}{3}$ in S. Robbii (Pl. X, 2), in which the corolla-tube is almost funnel-shaped, 3-4 $\mathrm{mm}$. wide at the mouth, and the broad lobes are spreading; it represents an extreme in this regard. In S. entebbensis, on the other hand, the tube is narrowly cylindrical throughout and the minute lobes are erect (see Pl. XII, 15), and there are many transitional forms between the two.

Further, minor points of specific difference, such as shape of leaves and stipules, etc., will appear in the descriptions of the species and in the key, necessarily more or less artificial, which follows. It will be realised that it is impossible to arrange the spccies in a single continuous series, such that each is between its two nearest allies. The order adopted is based, however, upon the suggested affinities as far as possible. 


\section{SYSTEMATIC ACCOUNT}

SabicEA Aublet, Pl. Guian. i. 192, t. 75, 76 (1775) ; Beritl. \& Hook. f. Gen. Pl. ii. 72. Schwenl.felda Schreb. Gen. 123 (1789); Willd. Sp. Pl. i. 982 (Schwenlifeldia).

Calycis limbi lobi saepius lineari-oblongi, plus minus elongati, raro subfoliacci, ampli, vel breves et obtusi. Corollae tubus saepius angustus insuper parum ampliatus; limbi lobi pro rata breves tubi dimidium nec attingentes, valvati. Stamina 4-5; antherae lineares, dorso afixae, obtusiusculae, inclusae. Discus annularis, crenulatus. Ovarium subglobosum, sacpius 4-5nonnunquam 2-loculare; stylus erectus, ramis $3-5$ raro 2 erectis linearibus obtusis; ovula in loculis numerosa, placentis tumidis ovarii axi affixis. Bacca varia, 3-5 raro 2-locularis, polysperma. Scmina minuta, ovoidea $v$. angulata, testa tenui, albumine carnoso.

Frutices saepius volubiles, raro repentes vel erecti, interdum suffrutices, rarissime arbores, pilosi, rarissime glabri. Folia opposita, rarissime verticillata. Flores sacpius inter minores, in capitulis vel cymis axillaribus.

Species 106, Madagascariac, Africae et Americae tropicae incolae.

\section{KEY TO THE SPECIES.}

A Erect shrubs, usually short, often subhcrbaccous. Leavos at least $20 \mathrm{~cm}$. long when mature. Stipules large, leafy, wholly or partly concealing tho donse inflorescenco, or the latter apparcntly caulifiorous. STIPULARIOPSIS.

Petiole over $20 \mathrm{cma}$. long (Amorican)

Pctiole less than $10 \mathrm{~cm}$. long (African).

Lateral leaf-veins usually 15 pairs at most; leaves narrow, sessile, oblanceolate to obovate

Lateral leaf-vcins usually more than 20 pairs.

Stipulcs $7 \mathrm{~cm} . \times 4 \mathrm{~cm}$.

Stipules less than $4 \mathrm{~cm}$. long, or at any rato nover so much as $2 \cdot 5 \mathrm{~cm}$. broad.

Calyx-lobes linear, subulate to filiform, or sotacoous. Corolla $1.5 \mathrm{~cm}$. long or less. Infloresconce compact.

Calyx-lobes hispid with long yollow hairs......... Calyx-lobes minutoly pubosccut

1. nmbrosa.

2. bicarpellata.

3. gigantostipula.

4. Urbaniana.

5. xanthotricha. 
Corolla $2.5 \mathrm{~cm}$. long or more.

Calyx-lobes about $1 \mathrm{~cm}$. long, flat, linear; inflorescence more or less compact

Calyx-lobes about $5 \mathrm{~mm}$. long, subulate-setaceous. Inflorescence lax ......................

Calyx-lobes ovate-lanceolate.

Stipules almost glabrous externally, without parallcl ribs .......................................

Stipules appressedly hairy externally, with prominent parallel ribs

3A. stipularioides.

8. geantha.

6. cauliflora.

7. Hierniana.

Scandent shrubs usually, with leaves rarely so much as $15 \mathrm{~cm}$. long (except S. Batesii). EUSABICEA.

$B$ Inflorescence more or less lax in mature flowering stage, its branches or the pedicels apparent; never a compact and involucrate pedunculate head (LAXX).

C Partial inflorescences without conspicuous oval or orbicular bracts.

D Fruiting calyx-lobes rarely exceeding $4 \mathrm{~mm}$., or if so, more or less setaceous.

E Leaves variously hairy, ncver felted, beneath.

Corolla-tube less than $15 \mathrm{~mm}$. long, usually $1 \mathrm{~cm}$. at most.

Hairs on mature corolla-tube dense, spreading...

Hairs on mature corolla-tube more or less scanty, adpressed.

Hairs on stem and leaves mostly adpressed, giving smooth, silky aspect; or scanty if spreading.

Bracts inconspicuous or obsolete, rarely manifest, then never connate.

Lateral leaf-veins distant, 12 pairs at most (America).

Erect shrub, 6-10 feet high

Climbing shrubs.

Corolla-tube barely $8 \mathrm{~mm}$. long at most.

Corolla-tube barely $6 \mathrm{~mm}$. long, lobes bearded at apex............

Corolla-tube $8 \mathrm{~mm}$. long, lobes not bearded at apex

Corolla-tube upwards of $1 \mathrm{~cm}$. long...

Lateral leaf-veins close, 15 pairs or more (Africa)

Bracts at base of inflorescence manifest, more or less connate at first.

Inflorescence remaining more or less compact; calyx-lobes not setaccous...

Infloresceuce becoming very lax; calyxlobes setaccous.............................

Hairs on stem and leaves dense, patent, giving shaggy aspect.

Climbing or straggling shrubs.

Peduncles barely as long as inflorescence at most ; bracts $5 \mathrm{~mm}$. long ...........

Peduncles 2-3 times as long as inflorescence; bracts over $15 \mathrm{~mm}$. long ......

[66. aspera.]

9. panamensis.

10. asperulc.

11. costaricensis.

12. paraënsis.

13. venosa.

31. colombiana.

32. umbellata.

27. Pearcei.

28. subinvolucrata. 
Small, erect, subherbaceous.

Corolla-lobes less than $2 \mathrm{~mm}$. long

Corolla-lobes over $4 \mathrm{~mm}$. long

Corolla upwards of $17 \mathrm{~mm}$. long. Calyx-limb shortly toothed.....................................

$E^{1}$ Leaves with manifest felt on lower surface (more or less deciduous in $S$. mollis and $S$. orientalis).

Calyx-lobes not subsetaceous (African, except $S$. erecta).

Bracts linear to lanceolate, usually inconspicuous or obsolete.

Corolla narrowly tubular throughout, slcnder, with minute erect lobes, and darkcoloured.

Corolla appreciably widened above, lobes usually patent, flowor white or greenishwhito.

Inflorescence remaining relatively compact, peduncle not manifest, or very short.

Calyx-lobes not exceeding $2 \mathrm{~mm}$. long ... Calyx-lobes reaching $4 \mathrm{~mm}$. or more. Foung parts soft with dense spreading hairs, branchlots not arachnoid ...

Young parts smooth with adpressed hairs.

Branchlets arachnoid, petioles rarely more than $1-1.5 \mathrm{~cm}$.

Ovary densely felted

Ovary hairy, not fclted

Branchlets not arachnoid,......... $2-3 \mathrm{~cm}$. or longer.

Inflorescence becoming lax, diffuse, pcdunculate

Bracts (2-3) broadly ovate, manifcst.

Climbing shrubs (Africa).

Petiole less than $2 \mathrm{~cm}$.; felt grey

Petiole $3.5 \mathrm{~cm}$. or more ; felt light brown.

Erect bush, $8 \mathrm{ft}$. high (Bolivia) .................

Calyx-lobes very varrow, subsetaccous (America).

Peduncle barely as long as the inflorescence.

Ovary arachnoid-folted

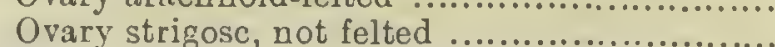

Peduncle 2-3 times as long as the inflorcsecnec

( $2 \mathrm{~cm}$. or morc), slender.

$D^{\prime}$ Fruiting calyx-lobes increasing to $7 \mathrm{~mm}$. long or morc, patent, more or loss rigid, and broadly linear to ovate-lanceolatc.

Stipules over $1 \mathrm{~cm}$. broad; involucrc manifest; long fruiting pedicels (Jamaica) .........................

Stipules much less than $1 \mathrm{~cm}$. broad.

Calyx-lobes ovate-lanceolate, involucre and peduncle manifest .................................

Calyx-lobes linear to narrow-lanceolate.

Lcaves not arachnoid bcneath.

Corolla more or less uniformly hairy externally, hairs adpressed.

Corolla 12-15 mun. (Mlcxico)

Corolla 18-20 mm. (Africa)
29. humilis.

30. Moorei.

14. laxa.

15. entebbensis.

13. venosa

[B. anzomala

16. mollis.

22. Smithii.

17. orientaiis.

18. longepetiolata.

19. discolor.

20. cameroonensis.

21. pseudocapitellata

23. erecta.

24. cuncata.

25. setiloba.

26. boliviensis.

33. hirla.

34. novo-granatensis

35. mexicana.

37. Schumanniana 
Corolla-tube almost glabrous externally.

Corolla-lobes patent, densely bearded, tube 8-9 mm. long

Corolla-lobes more or less erect, not densely bearded, tubs 10-15 $\mathrm{mm}$. long ............

Leaves with rather scanty but manifest arachnoid indurnentum beneath

${ }^{1}$ Inflorescence becoming very lax, diffuse, and compound; partial inflorescences associated with conspicuous broad bracts (FLORIBUND正).

Bracts suborbicular...

Bracts narrowly oral, or lanceolate.

$B^{1}$ Inflorescence and flowers closely sessile in mature flowering stage; or flowers rarely solitary (SEssiLes).

Calyx-lobes conspicuously subulato or setaceous, usually $5 \mathrm{~mm}$. long at loast.

Corolla $3 \mathrm{~cm}$. long or more.

Corolla $10 \mathrm{~cm}$. long, flowers solitary or subsolitary...

Corolla $3-4 \mathrm{~cm}$. long

Corolla less than $2 \mathrm{~cm}$. long.

Stipules entire; leaves mostly obovate, not lengthily acuminate; prostrate herb, rooting along stom.

Stipules laciniate, pectinate, or fimbriate; lcaves lanceolate to ovate, acuminate; erect or suberect shrubs, $6 \mathrm{ft}$. high or more (Madagascar and Mascarene Islands).

Leaves hairy above, never very unequal.

Learos subcoriaceous, lanceolate, branchlets pubescent, corolla $1 \cdot 3-1.5 \mathrm{~cm}$. long .........

Leaves submembranous, linear-oblong, branchlets arachnoid-glabrous, corolla $6.5 \mathrm{~mm}$. long

Leaves arachnoid to glabrous above, very unequal in upper twigs-appearing alternate

Calyx-lobes flat, appreciably broad.

Leaves verticillate, usually 3 in a whorl

Leaves opposite.

$F$ Calyx-lobes about $5 \mathrm{~mm}$. long, or more, in the flower, accrescent.

Stipules laciniate; leaves at each node very unequal

Stipules subentire; leares equal at each node, or almost so.

Leaves with manifost felt beneath.

Leaves arachnoid above, otherwise glabrous; bracts conspicuous, subinvolucrate.........

Leaves variously hairy, not arachnoid, above.

Bracts conspicuous, subinvolucrate.

Corolla exserted only a few $\mathrm{mm}$. beyond the erect calyx-lobes

Corolla exserted $1 \mathrm{~cm}$. beyond the erect calyx-lobes

Bracts not conspicuous in adult flower.

Fruiting calyx-lobes long setacco-acuminate

Fruiting calyx-lobes morc or less rigid, linear-oblong, not markedly asuminate.

Leavos not felted beneath
38. angolensis.

39. Vogelii.

36. Dewildemaniana.

103. floribunda.

102. segregata.

40. speciosissima.

41. speciosa.

42. medusula.

43. acuminata.

44. angustifolia.

45. diversifolia.

105. verticillata.

46. seua.

47. cinerea.

48. mollissima.

49. amazonensis.

50. pannosa.

51. grisea.

52. Burchellii. 
$F^{1}$ Calyx-lobes less, usually much less, than $5 \mathrm{~mm}$. long in the flower, and not mueh aeereseent.

Flowers not solitary.

Leaves with a light-coloured felt beneath.

Leaves subequal.

Infiorescenee not felted.

Corolla-tube $1 \mathrm{~cm}$. long, shaggy with long spreading hairs; young parts rufous ...

Corolia-tube $7 \mathrm{~mm}$. long at most, with scanty adpressed hairs.

Corolla-tube $7 \mathrm{~mm}$. long

Corolla-tube 3-5 $\mathrm{mm}$. long.

Cymes hairy; corolla-tube barely $4 \mathrm{~mm}$; small, erect, subherbaeeous

Cymes subglabrous; corolla-tube $5 \mathrm{~mm}$.

Infloreseence clothed with light-coloured felt.

Leaves hairy above, more or less rough.

Branchlets arachnoid (America).

Erect shrub; ealyx-lobcs ovate, not acuminate ...........................

Climbing; calyx-lobes lanceolatc, setaceo-acuminatc

53. velutina.

54. Lindmaniana.

55. camporum.

56. glomerata.

57. brasilicnsis.

59. guianensis.

Branchlets patcnt-pilose (Africa)

(Smithii.)

Leaves glabrous above, except sometimes on the midrib, often arachnoid.

Twining shrub; ovary 4-locular (Ameriea)

Tree; ovary bilocular (Africa)

Learcs at ench node very unequal (Africa).

Leaves hairy above (except in $S$. Mildbraedii var. glabreseens), mostly less than $15 \mathrm{em}$. long, stipulcs laciniatc.

Leaves elliptical or oval, $12 \mathrm{~cm} . \times 6.5 \mathrm{em}$. at most

Leaves obovate, reaching $15 \mathrm{~cm} . \times 8.5 \mathrm{~cm}$.

Leaves glabrous above, except midrib, $20 \mathrm{~cm}$.

long or more; stipules entire ..............

Leaves variously hairy, not felted, beneath.

Stipules broadly ovate, often rcflexed.

Corolla-tube over $1 \mathrm{em}$. long, with spreading hairs throughout

Corolla-tube less than $6 \mathrm{~mm}$., glabrate bclow, hairs adpressed above ........................

Stipules lanceolate, linear, or sctaccous, not reflexed.

Stipulcs lanceolate or linear-oblong.

Climbing shrub

Crccping undorshrub with prostrate rooting stem................................

Stipules setaceous.

Leaf acute at base (I ucatan)

Leaf rounded at basc usually (Africa)

Flowers solitary or subsolitary, witl 2 biacteoles closo under the ovary .............................

58. cana.

63. arborea.

\section{Mildbraedii.}

61. dubia.

62. Batesii.

64. glabrescens.

65. hirsuta.

66. aspera.

67. paria.

68. flagenioides.

69. parviflora.

104. bracteolata. 
$B^{2}$ Inflorescence a compact pedunculate head, invested by a conspicuous involucre (CAPYTATAs).

Leaves variously hairy, never felted, beneath.

Calyx-lobes linear to narrowly lanceolate, acuminate, acute, longer than the tube of the limb.

Hairs on calyx-lobes spreading, or if ascending, very dense.

Peduncle usually very short; at most $1.5 \mathrm{~cm}$.

Heads upwards of $2.5 \mathrm{~cm}$. in diameter.

Leaves with subcordate or rounded basc, petiole $1.5 \mathrm{~cm}$. at most .....................

Leaves with acute base, petiole elongate, to $3 \mathrm{~cm}$. or more.

Involucral bracts rarely exceeding $1 \mathrm{~cm}$. broad, longer than broad

Involucral bracts over $2 \mathrm{~cm}$. broad, broader than long ....................................

Heads not more than $1.5 \mathrm{~cm}$. in diameter.

Corolla-lobes very short..........................

Corolla-lobes half as long as the tube.............

Peduncle $2 \mathrm{~cm}$. long or more.

Climbing shrub, leaves about $12 \mathrm{~cm} . \times 5 \mathrm{~cm} . .$.

Prostrate herb, rooting along stem; leaves about $4 \mathrm{~cm} . \times 2 \mathrm{~cm}$.

Hairs on calyx-lobes adpressed closely, more or less scanty.

Peduncle $5 \mathrm{~mm}$. long

Peduncle $2-2 \cdot 5 \mathrm{~cm}$. long

Calyz-lobes shortly oblong, very obtuse, often spathu-

late, not longer than the tube of the limb, with closely adpressed hairs or glabrate.

Heads nearly $2 \mathrm{~cm}$. in diameter; peduncles very short

Heads less than $1 \mathrm{~cm}$. in diameter; peduncles over $1.5 \mathrm{~cm}$. long.

Peduncles hairy. Corolla-lobes $3-4 \mathrm{~mm}$. long or more ..............................................

Peduncles glabrate. Corolla-lobes $1.5 \mathrm{~mm}$. long....

Calyx-lobes ovate or ovate-lanceolate, or as much as

3-4 mm. broad, manifestly longer than tube.

Corolla-tube not more than $1.5 \mathrm{~cm}$. long.

Peduncles strigose-hairy, leaves mostly acute at base (America)

Peduncles glabrous or nearly so, leaves mostly cordate or rounded at base (Africa).

Climbing shrub $10-15$ feet high, flowers white... Creeping undershrub 1-2 feet, flowers pink ......

Corolla-tube $2 \cdot 5-3 \mathrm{~cm}$. long .

Calyx-lobes triangular or ovate, or broadly lanceolate, manifestly shorter than tube.

Involucre barely $1 \mathrm{~cm}$, in diameter (America) .........

Involucre over $2 \mathrm{~cm}$. in diameter; calyx-limb a toothed tube (Africa).

Climbing shrub; branchlets and peduncles with long spreading bairs .............................

Large tree; branchlets and peduncles glabrous ...

70. brevipes.

71. Gilletii.

72. tchapensis.

88. Talbotii.

89. fulva.

73. Schaeferi.

77. geophiloides.

95. Duparquetiana.

96. Robbii.

97. trigemina.

98. Laurentii.

99. Dinklagei.

80. mattogrossensis.

83. calycina.

84. Barteri.

78. pilosa.

79. Trailii.

100. Dewevrei. 101. gigantea. 
Leares with manifest felt on the lower surface (more or less deeiduous iu S. rufa).

Leaves not perfeetly glabrous above, at any rate not ou mid-rib; young leares hairy abovo.

Calyx-lobes narrowly oval (America)

Calys-lobes liuear, laneeolate, filiform, or minute (Africa).

Fruiting calyx-lobes manifest, at least 2-3 $\mathrm{nm}$. long.

Calyx-lobes not subfiliform; head rarcly more thau $2 \mathrm{~cm}$. in diameter in flowering stage.

Mature peduncles but sparsely hairy, or glabrate, or minutely pubescent, never felted.

Peduncles rarcly more than $2 \mathrm{~cm}$, rigid, sparsely bairy ; corolla hairy ............

Peduncles 3 em. or longer, glabrate, slender, curved; corolla glabrnus .................

Nature peduncles densely hairy, or felted.

Involucre over $1 \mathrm{~cm}$. deep; peduncle over $2 \mathrm{~cm}$.

Hairs on branchlets closely adpressed .... Branchlets densely clothed with spreading red hairs.

Iuvolucre not more than $7 \mathrm{~mm}$. deep.

Peduneles scldom so much as $1 \mathrm{~cm}$. long, calyx-lobes rarely so mueh as 3.5 mm. (sometimes $4 \cdot 5$ iu var.) ..........

Peduncles 5-6 cm. long, calyx-lobes accrescent to $7 \mathrm{~mm}$. in the fruit

Calyx-lobes subfiliform, olongate (over $1 \mathrm{~cm}$.)

eurling; head 3-4 cm. in diameter...........

Fruiting calyx-lobes minute (less than $1 \mathrm{~mm}$.),

donsely bearded

Leaves perfectly glabrous above, even on the veins; young leaves glabrcus or arachnoid, never hairy, above.

Peduncles and branchlets arachnoid-felted or glabrous.

Calyx-lobes short, tooth-like iu the flower, early strongly reflexed and adpressed to ovary .....

Calys-lobes lanceolate, not rcflexed.

Corolla arachnoid-glahrous extcrnally, fruits with conspicuous pedicels ......................

Corolla densely hairy externally, fruits subsessile

Peduueles and branchlets hairy, not felted

81. Trianae.

74. ingrata.

75. gracilis.

85. composita.

86. brannea.

87. capitellata.

90. Johnstonii.

76. ferruginen.

82. rufa.

91. pedicellata.

92. lanuginosa.

93. brachiata.

94. cruciata.

\section{SUBGENUS I. STIPULARIOPSIS Wernham.}

Frutices parvi saepe subherbacei nee scandentes; folin magna $20 \mathrm{~cm}$. longa v. longiora, stipulis magnis foliaceis; inflorescentia saepius dense conferta sessilis v. subsessilis multiflora, axillaris v. lateralis cauliflora, raro laxiuscula; ovarium sacpius biloeulare. 
1. S. umbrosa Wernham.

Suffrutex caule procumbente ca. 1-pedalis; foliis paucis magnis ellipticis, utrinque angustatis, ad ca. $27 \mathrm{~cm} . \times 15 \mathrm{~cm}$., subcarnosis, utrinque nisi subtus in venis pubescentibus glabris, venis secundariis utrinque $15-22$, petiolo ad $25 \mathrm{~cm}$. elongato, glabrato; floribus candidissimis in verticillis subsessilibus confertis, basibus a bracteis latis in setis plurimis ad $2 \mathrm{~cm}$. v. longioribus fimbriatis occlusis; corollae extus glabrae tubo insuper ampliato, ad $4 \mathrm{~cm}$. longo, lobis oblongis subacutis ca. $8 \mathrm{~mm}$. longis ( $\mathrm{Pl} . \mathrm{I}, 1)$.

Colombia: Antioquia, in the deep shade of the forest, at $3000 \mathrm{ft}$., Kalbreyer 1837 ! Hb. Kew.

Remarkable for the great length attained by the petiole. It is the only New World representative of the subgenus.

2. S. bicarpellata K. Schum., in Engl. Bot. Jahrb. xxxiii. 337 (1903).

Suffrutex erectus caule brevi simplice lignoso; foliis ad $22 \mathrm{~cm}$. $\times 6 \mathrm{~cm}$. oblanceolatis ad obovatis sessilibus, renis secundariis distantibus, stipulis majusculis; inflorescentia in foliorum axillis fasciculata; calycis totius $3 \mathrm{~mm}$. longi lobis subulatis ; corollae albae lobis $3 \mathrm{~mm}$. subulatis; ovario bicarpellato.

Cameroons: Bipinde, Zenker 1041! Kribi, Mildbraed 5905! Hbb. Mus. Brit., Kew., Berol., Boiss., Mus. Paris.

Notable for the long, crowded, sessile leaves, oblanceolate in contour, being gradually narrowed from the broad, rounded, distal portion to the base, with large leafy stipules.

3. S. gigantostipula K. Schum., in Engl. Bot. Jahrb. xxxiii. 337 (1903).

Suffrutex erectus ad 10-pedalis, caulibus paucis lignosis; foliis inter maxima, plerumque oblanceolatis, petiolatis, venis secundariis pluribus approximatis, stipulis maximis $7 \mathrm{~cm} . \times 4 \mathrm{~cm}$. inflores. centias subsessiles fasciculatas occludentibus ; calycis lobis oblongolanceolatis acutis ad $8 \mathrm{~mm}$. longis; corolla ad $2 \cdot 2 \mathrm{~cm}$. longa (Pl. XII).

S. Nigeria: Oban, Talbot 259! Cameroons : Bïsgen 451! Ledermann 595 ! Bipinde, Zenter 2474! Dinklage 1011! Hbb. Mus. Brit., Kiew., Berol.

The leaves and stipules attain the largest size of any in the genus, and their dense aggregation upon the shoot presents a striking appearance.

\section{A. S. stipularioides Wernham.}

Verisimiliter frutex erectus; foliis inter maximis obovatis vix acuminatis basi angustatis utrinque nisi subtus obscure in venis sparse sericeo-strigosis glabris, stipulis ovatis majusculis 
extus sparse pilosis; floribus pedicellatis in cymis alaribus multifloris arcte confertis ; calycis subglabri lobis linearibus complanatis acutis inter longiores; corollae extus glabrae tubo longiusculo insuper parum ampliato, lobis brevissimis late triangularibus (Pl. XII).

Cameroons: Batanga, Bates 423 ! Hb. Mus. Brit.

The specimen bears but one mature leaf, and this is about $40 \mathrm{~cm} . \times 15 \mathrm{~cm}$., with a rather woody stalk $7 \mathrm{em}$. long, and about 25-30 secondary veins on either side of the midrib. Stipules $: \cdot 5$ em. $\times 2 \mathrm{~cm}$. Calyx-lobes nearly $1 \mathrm{em}$. long; corolla-tube $2 \cdot 5 \mathrm{~cm}$. long.

Near S. gigantostipula K. Schum. but distinct in the shape of the calyx-lobes and size of the corolla (PI. XII).

\section{S. Urbaniana Wernham.}

Suffiutex erectus ad 8-pedalis, caule simplice lignoso; foliis inter maxima, venis secundariis pluribus approximatis, stipulis inter majores foliaeeis; inflorescentiis fasciculatis sessilibus cauliHoris; calycis lobis longis subulatis, pilis patentibus hispidis; corolla inter breviores; ovario bicarpellato (Pl. XII).

Fernando Po, 1950-3900 ft. : Mildbraed 7041 I Hb. Berol.

The stem is wore or less hispid with short spreading hairs, and glabrescent. The elliptic leaves are about $20 \mathrm{~cm}$. long and $14 \mathrm{~cm}$. broad, shortly acuminate, with $25-30$ pairs of lateral veins; lispid on both sides with rather sparse, spreading yellowish hairs, more densely on the margin and veins below. The midrib and petiole are densely hairy, the latter about 4-5 $\mathrm{cm}$. long. Stipules membranous, broadly lanceolate, $5.5 \mathrm{~cm} . \times 2 \mathrm{~cm}$, sparsely and locally pubescent on the outside. Flowers white, many together in globular sessile clusters. The subulate or setaceous ealyx-segments attain more than $1 \mathrm{~cm}$. in length, almost equalling the eorolla, with tube about $1.5 \mathrm{~cm}$. long, silliy pubeseent externally in the upper half, glabrate in the lower; lobes laneeolate, not much more than $1 \mathrm{~mm}$. long. (1913).

5. S. xanthotricha Wernham, in Cat. Talb. Niger. Pl. 42

Frutex erectus mediocris; foliis late ellipticis ad $45 \mathrm{~cm} . \times 18$ cm., venis secundariis utrinque 24 nec valde approximatis, stipulis ovatis $2 \cdot 7-3 \cdot 4 \mathrm{~cm} . \times 1 \cdot 2-2 \cdot 2 \mathrm{~cm}$. foliaceis ; inflorescentiis fascieulatis sessilibus caulifloris; calycis lobis ca. $1 \mathrm{~cm}$. setaeeis minute pubescentibus; corolla $1.5 \mathrm{~cm}$. longa extus glabra (Pl. $\mathrm{XI}, 1-4)$.

S. Nigeria: Oban, Talbot $249 !$ Hb. Mus. Brit.

\section{S. cauliflora Hiern, in Fl. 'Trop. Af 1 . iii. 77 (1877).}

Frutex erectus ; foliis inter majores, venis sccundariis utrinque ad ca. 23 , stipulis ca. $2.8 \mathrm{~cm} . \times 1 \cdot 5 \mathrm{~cm}$. extus glabratis nec 
parallele costatis ; inflorescentiis fasciculatis sessilibus caulifloris ; calycis lobis brevibus lanceolatis; ovario bicarpellato.

St. Thomas Island, 5000-8000 ft.: Mann! Hb. Kew. Moller 2 ! Hb. Berol.

Two specimens in the Kew herbarium bear the name $S$.? cauliflora, both collected by Mann, one in St. Thomas Island, the other ncar the Gaboon River. These appear to be quite distinct. In the former, to which I propose to assign the name $S$. cauliflora, the leaves are conspicuously hairy on both sides, while the ovate membranous stipules are almost glabrous when mature. The hairs on the young parts and petioles are spreading and more or less shaggy. It appears, moreover, to be a montane species, in contrast with the other, which occurs at no great elevation, and for which I propose the name S. Hicrniana.

\section{S. Hierniana Wernham.}

Frutex erectus; foliis coriaceis ad $37 \mathrm{~cm} . \times 18 \mathrm{~cm}$, utrinque nisi in venis fere glabris, venis secundariis utrinque ca. 24, stipulis $3 \cdot 5 \mathrm{~cm} . \times 1 \mathrm{~cm}$. sericeis, venis prominentibus pilosis parallelis ; inflorescentiis abbreviatis fasciculatis caulifloris; calycis lobis lanceolatis breviusculis ; ovario bicarpellato.

Gaboon River: Mann 918! Hb. Kew. Libreville, Klaine 1929! $\mathrm{Hb}$. Mus. Paris. Fernando Po, 600-800 ft. : Mildbraed 6288! Hb. Berol.

Rather less than $10 \mathrm{ft}$. high, leares obovate or oblanceolate, scarccly acuminate, glabrous cxcept for the silky veins seen from the lower surface, manifestly larger and of firmer texture than in the previous species. Petiole sparsely and appressedly hairy, about $2 \mathrm{~cm}$. long. Stipules tough, lanceolate, acuminate. Calyx-lobes 1-2 mm. long.

\section{S. geantha Hiern, in Fl. Trop. Afr. iii. 78 (1877).}

Frutex erectus 10-pedalis ; foliis ca. $28 \mathrm{~cm} . \times 13 \mathrm{~cm}$., glabris, renis secundariis utrinque ca. 25 , petiolo $3-4 \mathrm{~cm}$, stipulis ovatotriangularibus extus glabris $3-4 \mathrm{~cm} . \times 1 \cdot 5-2 \mathrm{~cm}$., acuminatis; inflorescentiis laxiusculis prope caulis basin lateralibus; florun pedicellis gracilibus ad $9 \mathrm{~mm}$.; calycis lobis subulatis $\mathrm{r}$. setaceis ca. 4-6 mm. longis ; corolla $1 \cdot 8 \mathrm{~cm} .-2 \cdot 5 \mathrm{~cm}$. longa extus glabra, lobis ovatis obtusis; ovario bicarpellato (Pl. XII).

Gaboon: Sierra del Crystal, Mann 1728! Hbb. Kew., Mus. Paris.

Remarkable for the complete glabrousness of the leares, which extends even to the reins, petioles, and stipules - a rare feature in this genus. 


\section{Subgends II. EUSABICEA Wernham.}

Frutices saepius scandentes raro erecti, rarissime herbae repentes $\mathrm{v}$. arbores; folia mediocra longitudine $15 \mathrm{~cm}$. nisi in sectione Floribundis nonnunquam vix ad $20 \mathrm{~cm}$. rarissime excedentia ; ovarium saepius 4-5-loculare, rarissime bicarpellatum.

\section{Sectio i. LAx Wernham.}

Inflorescentia in maturitate plus minus laxa, ejus ramulis vel pedicellis manifestis, nunquam capitulata involucrata necnon pedunculata.

\section{S. panamensis Wernham.}

Frutex erectus 6-10-pedalis ramulis dense et appresse flavosericeis; foliis ovalibus vix acuminatis apice valde obtuso ca. 7-8 cm. $\times 3 \cdot 5-4 \mathrm{~cm}$., supra sparsim longiuscule strigosis subtus pariter nisi in venis prominentibus sericeo-strigosis indutis, petiolo $1-1.5 \mathrm{~cm}$. longo, stipulis ovatis subacutis ca. $8 \mathrm{~mm} . \times$ $4 \mathrm{~mm}$. ; infloresccntia laxiuscula densissime flaro-strigosa $2-3 \mathrm{~cm}$. diam. attingente, pedunculo pro rata brevi (ca. 5-10 mm.), bracteis lanceolatis 2-3 connatis vix subinvolucrantibus nec saepe conspicuis; florilus candidis inter minores pedicello ad $8 \mathrm{~mm}$. v. longiore accrescente; calycis lobis linearibus demum patentibus vix $3 \mathrm{~mm}$. longis; corollae extus strigosae tubo ca. $6-8 \mathrm{~mm}$., lobis lanceolatis acutis $2 \cdot 5 \mathrm{~mm}$. longis apice barbato (Pl. XII).

Panama: Chagres, Fendler 181! Hb. Kew.

10. S. asperula Wernham. Manettia asperula Ball, in Journ. Linn. Soc. xxii. 142 (1886).

Frutex alte scandens ramulis dense sericeo-strigosis; foliis ovalibus breviuscule acuminatis $9-12 \mathrm{~cm} . \times 4-4.5 \mathrm{~cm}$., supra sparsim longiuscule strigosis, subtus pariter nisi in renis dense strigosis indutis, venorum lateralium paribus 10 raro excedentibus, stipulis latis ovatis v. subrotundis; inflorescentir laxiuseula ca. $1.5 \mathrm{~cm}$. diam. sericeo-strigosa, pedunculo ad $1 \cdot 5-2$ cill., bracteis paucis linearibus nec involucrantibus; calycis lobis ad 3-4 $1 \mathrm{~nm}$. saepius patentibus linearibus subsetaceis; corollne tubo extus sparsiuscule strigoso vix $6 \mathrm{~mm}$. longo, laciniis lanceolatis acutis $2.5 \mathrm{~mm}$. apice barbatis; bacca sparse patente piloso ca. $6 \mathrm{~mm}$. diam. a calycis laciniis ad $5 \mathrm{~mm}$. accretis coronata (PI XII).

Colombia: Shady places, coast near Bucnaventura, Ball! Hb. Kew.

A distinct species, characterised chiefly by its small corolla with bearded lobes. 


\section{S. costaricensis Wernham.}

Frutex verisimiliter subscandens, ramulis novellis dense flavosericeis deinde sparsim appressiuscule pilosis demum glabrescentibus; foliis ellipticis v. oblongis acuminatis ad ca. $12 \mathrm{~cm} . \times$ $4.3 \mathrm{~cm}$. apice subacuto, basi saepius subcordatis, supra nisi in venis saepius impressis strigillosis glabratis, subtus pariter nisi in renis prominentibus flavo-strigosis indutis, petiolo dense strigoso ad $1.5 \mathrm{~cm}$. longo, stipulis orali-oblongis $1 \mathrm{~cm} . \times 8 \mathrm{~mm}$. excedentibus, apice rotundato ; inflorescentia laxiuscula vix $2 \mathrm{~cm}$. diametro, bracteis paucis nec involucrantibus lanceolatis ad ovalibus, his acuminatis obtusis ad $7 \mathrm{~mm} . \times 4 \mathrm{~mm}$., pedunculo nec manifesto vel ad $5 \mathrm{~mm}$. longo; calycis lobis latiusculis oblongis flore vix $3 \mathrm{~mm}$. longis fere glabris; corollae extus sparsim strigillosae tubo cylindraceo vix $8 \mathrm{~mm}$. longo, lobis patentibus $2 \mathrm{~mm}$. longis apice nee barbatis; ovario dense flavo-strigoso (Pl. XII).

Costa Rica: Buenos Aires, in hedges, Pitticr 6712! and on the Savannah, Pittier 4025! Hbb. Mus. Brit., Kew., Boiss., Brux. Pittier 2904 ! (Hb. Nus. Brit.), from woods about the bay of Salinas, probably is referable to this species; but the specimen bears no corollas.

Near S. asperula, from which it is distinguished chiefly by the sizc and indumentum of the corolla; and $S$. colombiana, in which the bracts are much larger and subinvolucrate, and the calyx.lobes longer. The flower-buds in the present species are characteristic, being very stout, oblong or spindle-shaped, acuminate and subacute, and densely covered with yellow silky appressed hairs.

12. S. paraënsis W'ernham. S. umbellata Per's. var. paraënsis K. Schum., in Mart. Fl. Bras. VI. vi. 304 (1889).

Frutex scandens ramulis strigosis; foliis majusculis ca. 11$12 \mathrm{~cm} . \times 5 \mathrm{~cm}$. ovatis acuminatis, petiolo ca. $1 \mathrm{~cm}$. longo, venis 10-12 paribus subdistantibus supra sparsissime hirto-puberulis v. glabratis subtus pariter nisi in venis prominentibus appresse strigosis indutis, stipulis oblongo-ovatis ca. $1 \mathrm{~cm} . \times 6-7 \mathrm{~mm}$.; inflorescentia inter laxiores sparsiuscule strigosa, ad $2-3 \mathrm{~cm}$. longa $\times 4-5 \mathrm{~cm}$. lata, pedunculo $1-1 \cdot 5 \mathrm{~cm}$. longo, bracteis inconspicuis nec involucrantibus; calycis lobis flore vix $2 \mathrm{~mm}$. longis subsetaceis nec $3 \mathrm{~mm}$. excedentibus; corollae extus sparsiuscule strigosae tubo gracili ca. $1 \mathrm{~cm}$. longo insuper vix ampliato lobis erectis oblongo-linearibus vix $2 \mathrm{~mm}$. longis; bacca pisiformi sparse appresse strigosa (Pl. XII).

Brasil: Juruá Miry, Amazonas prov. Ule 5669! Peru: Sarayacu, Castelnau! Hbb. Kew., Berol., Deless., Mus. Paris.

The exinvolucrate inflorescence, and much larger flowers with smaller calyx-lobes, distinguish this species sharply from S. umbellata. The name and synonymy adopted above is based on the plant quoted, which bears the varietal name in Schumamn's own writing. According to the account in the Flora Brasiliensis (loc. cit.), the same species 
occurs in the vicinity of Parí (Martius), in Peru (Poeppig 1939) and also in Tenezuela; but I hare had no opportunity of examining the specimens in question.

13. S. venosa Benth., in Hook. Niger Fl. 399 (1849). S. Kolbeana Büttner, in Verh. Bot. Ter. Brand. xxxi. 78. S. affinis De Wild. in Ann. Mus. Congo, Sér. T. i. TT.

Frutex scandens ad 19-23-pedalis ramulis sparse strigosis; foliis ovato-oblongis acuminatis ca. $8-9 \mathrm{~cm} . \times 4-5 \mathrm{~cm}$, petiolo ad $2.5 \mathrm{~cm}$., supra scabrello-pubescentibus v. glabratis, subtus pariter nisi in venis approximatis 15 paribus v. plurimis strigosis indutis, stipulis ovalibus pro rata parvis vix $8 \mathrm{~mm} . \times 4 \mathrm{~nm}$.; floribus albidis v. dilute brunneis v. Havo-viridibus; inflorescentia laxiuscula ramulis strigosis $\mathrm{v}$. glabrescentibus nec $2-3 \mathrm{~cm}$. toto excedente, bracteis lanceolatis pleruncue obtusis ad $8-9 \mathrm{~mm}$. Iongis nec conspicnis nec involucrantibus; calycis lobis lanceolatis ad ovatis plerunque obtusis vix $4 \mathrm{~mm}$. attingentibus; corollae extus sparsiuscule strigillosae tubo $8-9 \mathrm{~mm}$. lobis lanceolatis acutis vix $2 \mathrm{~nm}$. ; baccu alba strigillosa glabrescente $7-8 \mathrm{~mm}$. in diam., succo rubro (Pl. XII).

Senegambia: Tarkandy, Heudelot 821! Sierra Leone: Afzetius! Don! Scott Elliot 5276! S'meathmann! Smythe 55! Liberia: Dinklage 2224! Irory Coast: Jolly 97 (in part)! Cameroons: Buea, Preuss 8724! Reder 1154! Yaunde, Zentier 675! 719! Congo: Quango I., Büttner 440 ! French Congo, damp places, Lecomte C 92 ! C 99 ! Bangala, Demeuse 261! Bumba, Pynaert 42 ! Sabuka, E. \& $M$. Laurent! Lukombc, etc., Kasai, in manihot plantations, Sapin Y 34! Leopoldville, Allard 146! Kisantu, Gillet 159! 357 ! 1390! Vanderyst! Djunia Valley, Gillet 2760! 2779! Hbb. Mus. Brit., Kew., Berol., Boiss., Brux., De Cand., Deless., Holm., Mus. Paris.

A variable species. S. Kolbeana, founded on Buttner 440 , appcars distinet at first sight on the grounds of differences in the indumentum and looser venation; but the transitional features dis. played by other cxamples of S. venosa lead me to regard Buittner's plant as no more than a form of $S$. venosa. I hare examined the type-specimens of $S$. affinis at Brussels, and they are clcarly of the same species as typical $S$. venosa. The adrent of morc matcrial may, howerer, reveal the presence of more than one species among the specimens available at present. The description on the label of E. \& M. Laurent's Sabuka plant-_ "petit arbre lc long des rivieres ..."-is suggestive; but the specimen as it stands is inseparable fropı $S$. venosa. Allard's Leopoldville plant bears, as a common name, the words "Modila Dila."

Var. anomala Wernham.

Foliis subtus discoloribus, lanugine laevi grriseo indutis, calycis lobis brevissinis.

Congo: Eala, Pynaert 515 (in part)*! Bouga, Sanga 11., Schlechter 12658! Liver-banks, Lolika, Mosscka, Chevalicr 5069! Hbb. Mus. Brit., Kew., Berol., Brux., De Cand., Nus. Paris.

\footnotetext{
* See S. pseudocapitcllata, no. 21.
} 


\section{S. laxa Wernham.}

Frutex scandens ramulis appresse strigosis; foliis fere ad $15 \mathrm{~cm} . \times 5 \mathrm{~cm}$. ellipticis saepe oblongis utrinque angustatis longiuscule acuminatis obtuso apice, utrinque nisi in venis sparsc appresse strigosis glabratis, petiolo saepius ad $4 \cdot 5 \mathrm{~cm}$. elongato, stipulis late triangularibus reflexis; inflorescentin laxa sparse appresse pilosa, pedunculo communi nec manifesto, bracteis lanceolatis inconspicuis nec involucrantibus; floribus candidis breviter pedicellatis; calycis limbi dentibus brevibus nec accrescentibus triangularibus acutis tubum nec superantibus; corollae extus densiuscule appresse sericeae tubo gracili inter longiores ad $2 \mathrm{~cm}$. v. longiore, lobis erectis acutis lanceolatis $3 \mathrm{~mm}$. longis (Pl. II).

Cameroons: Bipinde, Zenker 4020!4072! 4567! Lolodorf, Staudt $237 !$ Up to $2600 \mathrm{ft}$. Hlbb. Mus. Brit., Kew., Berol., Brux., De Cand. Deless., Mus. Paris.

Distinguished readily by the lax inflorescence, the long slender silky corolla and short calyx-teeth, and also by the elongated petioles and secondary veins more or less distant.

\section{S. entebbensis Wernham.}

Frutex scandens parvus ramulis strigosis mox glabratis; foliis ovalibus ca. $6-7 \mathrm{~cm} . \times 3-3 \cdot 5 \mathrm{~cm}$. breviter acuminatis apice subacuto, supra scabrello-pubescentibus subtus in venis strigoso-sericeis aliter densiuscule arachnoideo-lanuginosis, petiolo $1 \mathrm{~cm}$. longo, stipulis pro rata angustis ovato-lanceolatis $5-6 \mathrm{~mm}$. $\times 2-2 \cdot 5 \mathrm{~mm}$.; inflorescentia inter laxas strigillosa, bracteis nec conspicuis; calycis lobis inter brevissimos mox reflexis; corollae fuscae extus nisi insuper strigillosae glabratae tubo ca. $7 \mathrm{~mm}$. longo insuper nec ampliato, lobis minutiusculis erectis (Pl. XII).

Uganda: Intebbe, $3900 \mathrm{ft}$, Broun 296! Hb. Kew.

Distinct in the lanceolate stipules and the small dark-coloured flowers with short caly $\mathrm{x}$-lobes and tubular corolla with very small lobes.

16. S. mollis K. Schum. MS. S. venosa Benth. var. villosa K. Schum., in Ann. Mus. Congo, Sér. II. i. fasc. 2, p. 31 (1900).

Frutex scandens ramulis pilorum patentum indumento molli villosis nec arachnoideis; foliis ovalibus ad $8-9 \mathrm{~cm} . \times 4 \mathrm{~cm}$. basi saepius obtusis, apice obtuso, breviter acuminatis, petiolo villoso vix $1 \mathrm{~cm}$. longo, utrinque qua ramuli praesertim subtus in venis molliter villosis, subtus in juventute etiam minute arachnoideo-lanuginosis; inflorescentia molliter villosa inter laxiores tamen compactiuscula, pedunculo communi saepius manifesto, bracteis dum adsint lancoolatis nec conspicuis; calycis lobis lineari-oblongis $\operatorname{mox}$ ad $4 \mathrm{~mm}$. accrescentibus; corolla vix 
6- $\vec{i} \mathrm{~mm}$. longa pallide violacea extus nisi in lobis sparsim barbatis glabra, tubo insuper ampliato; bacen sparse pilusa globosa ad $1 \mathrm{~cm}$. dian., pedicello gracillimo ad $5 \mathrm{~mm}$. (Pl. XII).

French Congo: Ogooue, N'jobe, Thollon 104! Achouka, Dybowski 157! Congo: Bingila, woods, Dupuis! Bakuhu, Clacssens 113! Lusombo, Claessens 161! Kasai, Pogge 981! Hbb. Mus. Brit., Kew., Berol., Brux., Mus. Paris.

Dupuis' plant cited above is the type of K. Schumann's variety of S. venosa (supra). I have examined all the available material, and it seems clear that the indumentum, venation, and calyx-characters justify the complete separation of this plant from $S$. venosa, and its inclusion in the same spccies with Pogge's Kasai plant. The latter is S. mollis K. Schum. MIS.; and it is appropriately named, being readily identifiable by the soft velvety indumentum which covers the leares, branchlets, and inflorescence-axes.

\section{S. orientalis Wernham.}

Frutex scandens ramulis pilis appressis laevibus saepius etiam arachnoideis; foliis ovalibus $\mathrm{v}$. oblongis $7 \mathrm{~cm} . \times 3 \mathrm{~cm}$. ad $10 \mathrm{~cm} . \times 4.5 \mathrm{~cm}$. basi rotundatis v. obtusis, petiolo $1-1.5 \mathrm{~cm}$. nonnunquam ad $2.5 \mathrm{~cm}$. arachnoideo, acutis acuminatis, supra scabrello-pubescentibus, infra plus minus arachnoideo-lanu ginosis, stipulis late ovatis subacutis reflexis; inflorescentia laxiuscula inter tamen compactiores appresse pilosa pedunculo brevissimo v. saepius occulto v. obsoleto, bracteis inconspicuis; floribus albis primo brevissime pedicellatis ; calycis lobis ad $3-4 \mathrm{~mm}$. lanceolatis acutis acuminatis; corollae extus sparse strigosae tubo insuper ampliato vix $1 \mathrm{~cm}$., lobis erectis anguste lanceolatis ca. $2 \mathrm{~mm}$. longis (PI. T, 2-5).

Congo: Mongala R., thickets, $1430 \mathrm{ft}$., Thonner 202! German E. Africa : Kilimanjaro, Derema, Volkens $133 !$ E. Usambara, Engler 675 ! 676! 709! Amani, Braun 1936! Zimmermann 40!107! Ulugurn, Goetze 209! Stuhlmann 8872! Hbb. Mus. Brit., Kew., Berol., Boiss., Brux.

The locality of Thonner's Congo plant is very isolated from the rest, and the flowers are said to be "jaunes, rougeâtres à l'extericur." It may be a western variety, but I can find no eritical characters in the available material to separate it from the East Afriean speeies.

18. S. longepetiolata De Wild,, in Ann. Mus. Congo, Sér. V. i. $78(1903-1906)$.

Suffrutex scandens ramulis laeribus sericeo-strigosis nec arachnoideis, demum glabris ; foliis ovalibus $6-7 \mathrm{~cm} . \times 3-3 \cdot 5 \mathrm{~cm}$. acuminatis basi saepius acutis, supra scalrello-pubescentibus subtus arachnoideo-tomentosis, petiolo graciliad $2-3 \mathrm{~cm} . \mathrm{v}$. longiore, stipulis oratis acutis; inflorescentia laxiuseula inter tamen compactiores strigosa saepius subsessili, bractcis haud manifestis; 
calycis lobis lanceolatis ad $4 \mathrm{~mm}$. attingentibus; corolla inter breviores tubo insuper ampliato; bacca albida succo purpureo.

Congo: Kimuenza, Gillet 2179! Mildbraed 3701! Lukolela, Kriekels! Hbb. Berol., Brux.

19. S. discolor Stapf, in Journ. Linn. Soc. xxxvii. 105 (1905).

Frutex volubilis alte scandens, ramulis breviter plus minus appresse pubescentibus demum glabris; foliis ovalibus ca. $9 \mathrm{~cm} . \times 4.5 \mathrm{~cm}$. acuminatis subacutis basi saepius subrotundis, petiolo elongato ad 2-4 cm., supra scabrello-pubescentibus, subtus argenteis dense araneoso-tomentosis, venis utrinque 12-16 conspicuis nonnunquam dilute roseis, stipulis ovatis acuminatis; cymis plus minus appresse hirsutis demum inter laxissimas, bracteis lanceolatis ca. $5 \mathrm{~mm}$. longis, pedunculo manifesto ad $1 \cdot 5-2 \mathrm{~cm}$. longo; calycis lobis ovato-lanceolatis obtusis flore vix $1.5 \mathrm{~mm}$. nec unquam ca. $2 \cdot 5 \mathrm{~mm}$. excedentibus; corollae saepe roseae extus sparsissime hirsutae tubo insuper leniter ampliato $8 \mathrm{~mm}$. longo, lobis triangularibus acutis patentibus ca. $2 \mathrm{~mm}$. longis, bacca albida (Pl. XII).

Liberia: Sinoe Basin and Momrovia, Whyte! Dintlage 2188! 2476 ! Gola, Bunting! Mt. Barclay, Bunting 28! Ivory Coast: Borobo, Chevalier 17664! Bingerville, Chevaliev 15343! Gold Coast: Murphy $679 !$ Hbb. Mus. Brit., Kew., Berol., Mus. Paris.

Val. ß. laxothyrsa Wernham.

Planta pilis densioribus longioribus patentibus; foliis majoribus ad $12 \mathrm{~cm} . \times 5 \mathrm{~cm}$; inflorescentia saepius laxiore, floribus paucioribus.

Liberia: Grand Bassa, in moist soil, Dinklage 1902! 1903! Hbb. Mus. Brit., Kew., Berol.

\section{S. cameroonensis Wernham.}

Frutex scandens ramulis pubescentibus junioribus araneosis ; foliis ellipticis ca. $8 \mathrm{~cm} . \times 4 \mathrm{~cm}$. vix acuminatis acutis, petiolo vix ad 1.5 cm. longo, supra asperulo-pubescentibus, subtus in venis sericeo-strigosis aliter dense araneoso-velutinis, stipulis late ovalibus reflexis ; inflorescentia dense sericea laxiuscularum inter compactiores, pedunculo ca. $5 \mathrm{~mm}$. longo, a bracteis manifestis late ellipticis subinvolucrantibus ca. $3 \mathrm{~mm} . \times 2.5 \mathrm{~mm} .2-3$ subtensa; calycis lobis lanceolatis nec setaceis, nec $3 \cdot 5 \mathrm{~mm}$. excedentibus; corollae tubo extus infra glabrato insuper sparsiuscule strigoso $7-8 \mathrm{~mm}$. longo, lobis extus appresse albido-sericeis lanceolatis ca. $1 \mathrm{~mm}$. longis (Pl. XII).

Cameroons: Molundu, Mildbraed 4711! $3000 \mathrm{ft.} \mathrm{Hb.} \mathrm{Berol.}$ 


\section{S. pseudocapitellata Wernham.}

Frutex scandens ramulis novellis dense flavo-sericeis nec araneosis tarde glabrescentibus; foliis late ellipticis v. oblongis ca. $10 \mathrm{crn} . \times 5.5 \mathrm{~cm}$., breviter acuminatis acutis basi saepius angustatis, supra sparsim hirtis, subtus discoloribus lanugine ininuto denso dilute brunneo indutis, venis subtus conspicuis fuscis sericeis utrinque secundariis 11-14, petiolo subelongato ad $3.5 \mathrm{~cm}$., stipulis late ovatis acutis reflexis ca. $5 \mathrm{~mm}$. $\times 5 \mathrm{~mm}$. floribus in cymis laxiorum inter compactiores multifloris, bracteis paucis ovatis ad $7 \mathrm{~mm} \times 3 \cdot 5 \mathrm{~mm}$. praesertim in juventute subinvolucrantibus demum deciduis, pedunculo ad 8-9 $\mathrm{mm}$. dense sericeo ; calycis lobis lineari-lanceolatis ad $4 \mathrm{~mm}$. longis; corollae tubo gracili $7.5 \mathrm{~mm}$. extus infra glabro insuper strigilloso, lobis lanceolatis $1 \mathrm{~mm}$. longis; ovario dense flavo-sericeo.

Congo: Eala, Pynaert 515 (in part)! Hb. Brux.

With the general appearance of $S$. capitellata, this species is readily distingrished by the inflorescence, which is not a close capitulum with an involucre. The nearest affinity is apparently S. camcroonensis, which differs in the indumentum of the leaves and branchlets, and in the short petioles.

\section{S. Smithii Wernham.}

Frutex volubilis ramulis gracilibus patente pilosis necnon arachnoideis; folits ellipticis ad $7 \cdot 5-8 \mathrm{~cm} . \times 3 \cdot 5-4 \mathrm{~cm}$. utrinque acuminatis acutis, supra asperulo-pubescentibus subtus dense broriter araneoso-tomentosis griseo-discoloribus, venis secundariis utrinque ca. 12-15, petiolo $5 \mathrm{~mm}$., stipulis ovatis subobtusis extus insuper glabrescentibus intus glabris ca. $5 \mathrm{~mm} . \times 4 \mathrm{~mm}$. mox reflexis; florilus saepe in fasciculis alaribus sessilibus confertis araneosis, inflorescentia nonnunquam laxescente; calycis lobis oblongo-linearibus ad ca. $4 \mathrm{~mm}$, longis subacutis, nisi in margine ciliatis glabratis demum plus minus reflexis; fructu sparse piloso necnon araneoso (Pl. XII).

Congo: Chr. Smith $59 !$ Hbb. Mus. Brit., Kew.

The phyletic position of this species is notable, on account of its transitional position between Sessiles and Laxæ (see p.16, and Fig. 3).

\section{S. erecta Rusby MS.}

Frutex 8-pedalis ramulis denso patente hirsutis ; foliis suborbicularibus ad ellipticis $\mathrm{v}$. ovato-oblongis $5.5 \times 4 \mathrm{~cm}$. ad $11 \times$ $5 \cdot 5 \mathrm{~cm}$., supra hispidulis subtus densiuscule al'aneoso-lanuginosis, petiolo ad $1.5 \mathrm{~mm}$. longo, stipulis late ovatis mox reflexis ca. $6 \mathrm{~mm} . \times 6 \mathrm{~mm}$.; inflorescentia laxiuscularum inter confertiores subglobosa dense patente-pilosi, pedunculo ad ca. 2 cm. longo, bracteis 1-2 suborbicularibus manifestis acutis $7 \times 5 \mathrm{~mm}$. nec involucrantibus; calycis lobis lanceolatis acutis ad $3-3.5 \mathrm{~mm}$. 
nec subsetaceis; corollae extus strigosae tubo ca. 3-4 mm., lobis lanceolatis $2 \mathrm{~mm}$. longis (Pl. XII).

Bolivia: Tumupasa, Williams 446! 590! $1800 \mathrm{ft}$. Hbb. Mus. Brit., Kew.

Notable for its erect habit, rather compact pedunculate inflorescence, and small corolla.

24. S. cuneata Rusby, in Mem. Torr. Bot. Club, vi. 47 (1896).

Frutex verisimiliter scandens ramulis griseo-araneosis; foliis 10-12 cm. $\times 4-4.5 \mathrm{~cm}$. ellipticis utrinque acutis acuminatis, supra asperulo-pubescentibus, subtus breviter araneoso-lanuginosis discoloribus, petiolo nonnunquam elongato ad ca. $2 \cdot 5 \mathrm{~cm}$., stipulis ovatis $7 \mathrm{~mm} . \times 5 \mathrm{~mm}$.; inflorescentia inter laxiores, bracteis linearibus ca. $5 \mathrm{~mm}$. longis nec conspicuis, ramulis strigosis araneosis, subsessili vel brevi pedunculo ralido ad ca. 5-8 mm. longo, pedicellis $3-5 \mathrm{~mm}$.; calycis lobis anguste linearibus subsetaceis ca. $5 \mathrm{~mm}$. longis; corollae purpureae pilosae $1 \mathrm{~cm}$. longae tubo insuper.infundibulari; ovario extus griseo-araneoso-lanato (Pl. XII).

Bolivia: between Guanai and Tipuani, Bang 1380! Hbb. Mus. Brit., Kew., Berol., Boiss., Deless.

The white felt on the under side of the leaves and on the ovary, and the almost setaceous calyx-lobes, make this species readily identifiable.

\section{S. setiloba Wernham.}

Frutex scandens 10-15-pedalis ramulis dense breviter cinereovillosis; foliis ellipticis $9-11 \mathrm{~cm} . \times 4-4 \cdot 5 \mathrm{~cm}$. utrinque angustatis acutis, petiolo brevi ad $10-12 \mathrm{~mm}$., supra in siccitate nigricantibus hispiclulis, subtus dense araneoso-lanuginosis discoloribus, stipulis ovatis reflexis; inflorescentia inter laxiores tamen confertiuscula maxime vix $1.5 \mathrm{~cm}$. metiente patente pilosa, pedunculo valido ad $5 \mathrm{~mm}$. dense tomentoso nec cymam excedente, bracteis nec conspicuis nec involucrantibus; calycis lobis subsetaceis ad 3-4 mm.; coroll « tubulosa extus strigosa vix $1 \mathrm{~cm}$. longa lobis subminutis; ovario et fructu candido sparse piloso nec araneoso.

Colombia: Santa Cruz, in woods, Pearce! Hb. Mus. Brit.

\section{S. boliviensis Wernham.}

Frutex scandens caule juniore moliiter dense tomentoso demum glabrescente; foliis elliptico-lanceolatis ad ca. $15 \mathrm{~cm} . \times$ $5 \mathrm{~cm}$. utrinque angustatis acutis, petiolo $10-15 \mathrm{~mm}$. longo, supra molliter pubescentibus in siccitate viridibus subtus cinereis araneoso-lanatis discoloribus, stipulis majusculis late ovatis ca. $8 \mathrm{~mm}$. $\times 7 \mathrm{~mm}$. acutis nox reflexis; inflorescentia laxarum inter compactiores dense hispidulo-pubescente, pedunculo gracili 
pubescente longiusculo cymam 2-3-plo saltem excedente $(2 \mathrm{~cm}$.), bracteis paucis linearibus ad $8-9 \mathrm{~mm}$. nec involucrantibus; calycis lobis angustis subsetaceis ca. $4 \mathrm{~mm}$; ovario extus hispidulo. piloso.

Bolivia: Yungas, Bang $384 !$ Hbb. M[us. Brit., Boiss.

\section{S. Pearcei Wernham.}

Frutex scandens ramulis dense molliter tomentosis demum glabrescentibus; foliis elliptico-lanceolatis longiuscule acuminatis acutis basi saepius angustatis, petiolo brevi raro $1 \mathrm{~cm}$. excedente, utrinque praesertin subtus in venis patule hispidulo-hirsutis nec araneosis, stipulis late ovatis majusculis ca. $11 \mathrm{~mm} . \times 7 \mathrm{~mm}$.; inflorescentia laxarum inter compactiores ramulis pedunculoque brevi $5-8 \mathrm{~mm}$. cymam nec superante hispidulis, bracteis lanceolatis raro $5 \mathrm{~mm}$. excedentibus nec involucrantibus; calycis lobis angustis subsetaceis $4-5 \mathrm{~mm}$. demum attingentibus, bacca alba pedicello $5 \mathrm{~mm}$. v. magis longo (Pl. LII, 1 ).

Colombia: Woods about Noro, 3000-4000 ft., Pearce! Hb. IIus. Brit.

\section{S. S. subinvolucrata Wcrnham.}

Herba volubilis caule suffrutescente primo densiuscule tomentoso demum glabrescente; foliis ellipticis utrinque angustatis nec vix acuminatis subacutis $10-11 \mathrm{~cm} . \times 4 \cdot 5 \mathrm{~cm}$., supra nolliter hispidulo-pubescentibus subtus in venis densiuscule strigosis nee araneosis nec aliter indutis, petiolo brevissimo $7 \mathrm{mn}$., stipulis late ovatis $8-9 \mathrm{~mm} . \times 7-8 \mathrm{~mm}$. mox deflexis; inflorescentia laxarum inter compactissimas subcapitata hispidulo-tomentosa, pedunculo $2-3 \mathrm{~cm}$. longo cymain 2-3-plo superante, bracteis $2-3$ foliosis lanceolatis ad $17 \mathrm{~mm} . \times 5.5 \mathrm{~mm}$. subinvolucrantibus; calycis lobis lineari-lanceolatis v. linearibus nec $4 \mathrm{~mm}$. excedentibus; corolla vix 7-8 mm. longa extus sparsiuscule strigosa alba (Pl. III, 2, 3).

Eastern Peru : near Tarapoto, Spruce $4370 !$ Hb. Kew.

29. S. humilis S. Moore, in Trans. Linn. Soc. Ser. II. iv. 369 (1893).

Herba caulibus plurimis subfastigiatis a rhizomate brevi valido lignoso ascendentibus densissime villosulis foliosis 20$25 \mathrm{~cm}$. longis ; foliis late ellipticis ad subrotundis ad $6-8 \mathrm{~cm} . \times$ $3.5 \mathrm{~cm}$., brevissime petiolatis, hispido-hirsutis nec araneosis vix v. brevissime acuminatis, stipulis ovatis ca. $7-9 \mathrm{~mm} . \times 5 \mathrm{~mm}$. nec reflexis; inflorescentia pilosissima subsessili $v$. pedunculo brevissimo compacta demum laxescente, bracteis ovatis acutis acuminatis $5-6 \mathrm{~mm} . \times 3 \mathrm{~mm}$. vix inrolucrantibus; calycis lobis lanceolatis $3 \mathrm{~mm}$. longis; corollae albac tubo extus nisi insuper 
in parte brevi ampliato puberulo glabro, $6 \mathrm{~mm}$. longo, lobis $5-7$ extus puberulis acutis lanceolatis vix $2 \mathrm{~mm}$. longis (Pl. XII).

Brasil: Matto Grosso, Santa Cruz, Spencer Moore 472! S. Anna da Chapada, Malme 2071! Minas Geraes, Quartel de Biribiry, Glaziou 19420a! Hbb. Mus. Brit., Kew., Berol., Holm., Mus. Paris.

Remarkable for the lowly erect habit (see Introduction) and the very dense shaggy covering of the stem, leaves, etc. The latter are relatively very broad and almost sessile. The true characters of the inflorescence are apt to be masked by the hairiness.

Var. lanceolata S. Moore, loc. cit.

Major, foliis elliptico-lanceolatis $6.5 \mathrm{~cm} . \times 2 \cdot 7 \mathrm{~cm}$. ad $9 \mathrm{~cm}$. $\times 3 \cdot 2 \mathrm{~cm}$., minus pilosis; floribus parum majoribus.

Brasil : Matto Grosso, Santa Cruz, Speneer Moore 794! Cuyaba, Malme 2684! Hbb. Mus. Brit., Kew., Berol., Holm.

\section{S. Moorei Wernham.}

Herba caulibus plurimis basi suffiutescentibus, e rhizomate brevi lignoso valido ascendentibus densissime villosulis desuper pubescentibus 1-pedalibus; foliis ellipticis ad obovato-oblongis $8 \mathrm{~cm} . \times 3.5 \mathrm{~cm}$. ad $11 \mathrm{~cm} . \times 5.5 \mathrm{~cm}$. subsessilibus utrinque plus minus patule sericeo-strigosis nec araneosis, stipulis ovatis breviter acuminatis acutis $8-9 \mathrm{~mm} . \times 6-7 \mathrm{~mm}$. nec deflexis; inflorescentia pauciflora subsessili compactiuscula demum laxescente, bracteis lanceolatis ovatisque ad ca. $6 \mathrm{~mm}$. longis nec involucrantibus; calycis lobis lanceolatis ad $3-4 \mathrm{~mm}$. longis; corollae insuper extus sparse strigillo-villosae tubo anguste infundibulari $6-7 \mathrm{~mm}$. longo lobis lanceolatis ad $5 \mathrm{~mm}$. longis (Pl. IV, 1-4).

Brasil : Matto Grosso, Santa Anna da Chapada, Robert 687! Hbb. Irus. Brit., Kew.

Appears like a stout form of S. humilis, which it resembles in habit, but is readily distinguished by the shape of the leaves and the shape and size of the corolla.

\section{S. colombiana Wernham.}

Frutex ramulis validiusculis junioribus strigosis vel strigillopubescentibus; foliis ovalibus ad ca. $10 \mathrm{~cm} . \times 5.5 \mathrm{~cm}$., utrinque angustatis, utrinque nisi subtus in venis densiuscule sparsissime strigosis v. glabratis, petiolo ad 1-1.5 cm., stipulis majusculis 7-10 mm. $\times 7-8 \mathrm{~mm}$. mox reflexis ovatis obtusis; inflorescentia sparse strigosa laxiorum inter compactas nec multo laxescente subsessili v. pedunculo saepius vix ad $7-8 \mathrm{~mm}$., raro ad $1.5 \mathrm{~cm}$. longo, bracteis $2-3$ manifestis ovato-lanceolatis longe acuminatis ad $7-8 \mathrm{~mm}$. longis, primo basi connatis subinvolucrantibus; pedicellis ad 6-7 mm.; calycis lobis lineari-oblongis glabris ad 
$4.5 \mathrm{~mm}$. longis, corollae albae extus sparsiuscule strigosae tubo infundibulari-cylindraceo $9 \mathrm{~mm}$. longo lobis lanceolatis acutis $2 \cdot 5 \mathrm{~mm}$. longis (Pl. XII).

Colombia: Triana 681! Smith 1834 (in part)*! Ocaña, 3900 ft., Schlim 697! Cauca, 4000 ft., Triana 1752! Chiriqui lagoon, Hart 144! Venezuela: Carabobo, 4000 ft., Functic of Schlim 621! $3000 \mathrm{ft} .$, Linden 14:8! Hbb. Mus. Brit., Kew., Cantab., Berol., Boiss., Brux., De Cand., Holm., Mus. Paris.

A somewhat difficult species, on aecount of the indiffercnt preservation and immature eondition of much of the matcrial. Linden's 1498 may be regarded as the type; and Triana 1752, as to be seen in the English herbaria, differs so much from Linden's speeimen that I took it originally for a distinct species with inconspicuous bracts and short reflexed calyx-lobes. The Paris and Brusscls spceimens of Triana 1752, however, leave no doubt that this plant is to be referred to $S$. colombiana.

This spccies is nearly related to the Peruvian S. umbellata, but distinet in the eharactcrs of the inflorescence and calyx-lobes, and the sinaller, less acuminate leares witl shorter petioles.

32. S. umbellata Pers. Syll. i. 203 (1805). Schwenlifellia umbelluta Ruiz \& Pavon, Fl. Per. \& Chil. ii. 55, t. 200, a. (1789).

Frutex volubilis ramulis sparse strigosis nisi insuper scabridulis mox glabrescentibus; foliis ovalibus ca. $14 \mathrm{~cm} . \times 6 \mathrm{~cm}$. longiuscule acuminatis, petiolo $2-\hat{3} \mathrm{~cm}$, utrinque nisi subtus in venis spar'se strigosis glabratis, stipulis ovatis ad $16 \mathrm{~mm} . \times 10 \mathrm{~mm}$; infiorescentia primo capitata involucrata demum valde laxescente, bracteis 2-3 manifestis ovatis v. lanceolatis $10 \mathrm{~mm}$. $\times 5 \mathrm{~mm}$. vix acuminatis, pedunculo ut ramuli strigoso ca. $1 \mathrm{~cm}$. cymam ncc superante; calycis lobis subsetaceis ad $5 \mathrm{~mm}$. v. magis accrescentibus incurvatis; corollce inter minores tubo extus desuper glabro insuper strigroso (Pl. XII).

Peru: Ruiz \& Pavon! Pocppig 43! 1226! Matthews 1951! Richard! Cochero, Dombey 561! Cavanilles! Hbb. Mus. Brit., Kiew., Cantab., Berol., Boiss., De Cand., Dcless., Nus. Matrit., Mus. Paris.

Essentially a Peruvian species; distinet in the infloreseence, whiel is a pedunculate involucrate head when joung, but which becomes very lax and diffuse at the early fruiting stage; and also in the setaceous calyx-lobes.

33. S. hirta Sw. Prod. Veg. Ind. Occ. 46 (17SS). Schwentfelda hirta Sw. Fl. Ind. Occ. i. 450. Schwenlifeldia hirta Willd. Sp. Pl. i. 982.

Frutex altus ramulis subscandentibus sparse patente hirsutis ; foliis ellipticis utrinque angustatis, petiolo ad "2 $\mathrm{cm}$, utrinque

$$
\text { * See Sp. } 65 .
$$


praesertim subtus in venis hirto-pilosis nec araneosis, stipitis majusculis rotundis ad $10 \mathrm{~mm} . \times 14 \mathrm{~mm}$.; inflorescentia demum laxescente umbellata, pedunculo ad $14 \mathrm{~mm}$. longo, bracteis manifestis $2-3$ oratis $1 \mathrm{~cm}$. longis subinvolucrantibus, pedicellis in fructu 6-9 mm.; calycis lobis saepius ovato-lanceolatis ad $7 \mathrm{~mm}$. longis; corollae albae extus sparsissime pilosae tubo anguste infundibulari $1 \mathrm{~cm}$. v. magis longo, lobis ovato-lanceolatis $4 \mathrm{~mm}$. longis; bacca alba (PI. XII).

Jamaica: Macfadyen! Masson! Purdie! Swartz! Wright 655! Fl. Jam., Harris 9246! 10278! 10561! Wilson! Bertero 2703! Hbb. Mus. Brit., Kew., Berol., Deless., Holm., Krug \& Urban, Mus. Paris.

This species has been confused with S. hirsuta; but it seems to be quite distinct, not only in the characters of the inflorescence, but in the size and shape of the corolla, and the rery ample calyx-lobes.

34. S. novo-granatensis K. Schum., in Mart. Fl. Bras. vi. pt. 6, 303 (1889). S. cinerea Karst. non Aubl. ex K. Schum. loc. cit.

Foliis lanceolatis ad ovalibus $12-13 \mathrm{~cm} . \times 4-6 \cdot 5 \mathrm{~cm}$, supra sparsim aspero-strigillosis, subtus in venis pilosis, apice obtusissimo, basi acutis, petiolo ad $2 \mathrm{~cm}$., stipulis ovalibus $7 \mathrm{~mm} . \times 5 \mathrm{~mm}$. ad $1 \mathrm{~cm} . \times 1.2 \mathrm{~cm}$; inflorescentia laxiuscula, bracteis ovatis foliaceis modo S. hirtae subinvolucrantibus, pedunculo $2-2 \cdot 5 \mathrm{~cm}$. longo densissime flavo-hispido, pedicellis longiusculis ; calycis lobis late lanceolatis ad $6 \mathrm{~mm} . \times 2.5 \mathrm{~mm}$. patente hirsutis; ovario densissime sericeo-piloso; corollae extus dense hirsutae tubo gracili $7 \mathrm{~mm}$., lobis vix $2 \mathrm{~mm}$. long.

Colombia: Guaduas, Karsten! Hb. Berol. Bogota, Susumino, $3250 \mathrm{ft}$., Triana 1755 ! in hb. Mus. Paris. Bogota, Triana! in hb. Cantab.

\section{S. mexicana Wernham.}

Suffrutex scandens ramulis appresse pilosis ; foliis lanceolatis ad ellipticis utrinque ang ustatis, $8-12 \mathrm{~cm} . \times 2 \cdot 5-5 \mathrm{~cm}$. longiuscule acuminatis acutis, utrinque sparsiuscule hirto-pilosis, venis subtus strigosis approximatis utrinque 15 , petiolo ad ca. $1 \mathrm{~cm}$., stipulis late ovatis $8 \mathrm{~mm} . \times 10 \mathrm{~mm}$. acuminatis acutis; inflorescentia inter laxiusculas necnon compacta strigosa subsessili, bracteis nec manifestis; calycis lobis patente pilosis linearibus saepe setaceoacuminatis $6-10 \mathrm{~mm}$. longis; corollae albae extus uniforme strigosae tubo $10-12 \mathrm{~mm}$., lobis lanceolatis acutis $3 \mathrm{~mm}$. longis (Pl. V, 1, 2).

Mexico: Licbmann 43! Oaxaca, $3000 \mathrm{ft}$. Galeotti 2662 ! $3000 \mathrm{ft}$. Hbb. Kew., Berol., Brux., Deless., Mus. Paris.

Interesting as the sole representative of the genus in Mexico. 


\section{S. Dewildemaniana Wernham.}

Frutex 3-4-pedalis ramulis gracilibus novellis dense sericeostrigosis tarde glabrescentibus, caule basi diffuse radicante, in siccitate nigricante; foliis ovato-lanceolatis ca. $10.5 \mathrm{~cm} . \times$ $4.5 \mathrm{~cm}$., longiuscule acuminatis acitis basi subrotundatis $v$. obtusis, petiolo ad $1 \mathrm{~cm}$., supra sparsim hirtellis, subtus sparsim araneosis, stipulis ovatis acutis acuminatis ad $7 \cdot 5 \mathrm{~mm} . \times 5 \mathrm{~mm}$. mox reflexis; cymis subumbellatis ca. $2 \cdot 5 \mathrm{~cm}$. diametro paucifloris laxiusculis, pedunculo $5 \mathrm{~mm}$. longo, bracteis manifestis saepius 2 lanceolatis acuminatis acutis ca. $1 \mathrm{~cm} . \times 4 \mathrm{~mm}$., extus sparse pilosis intus glabris, pedicellis gracilibus ad $4 \mathrm{~mm}$.; calycis lobis lanceolatis acutis ad $7 \mathrm{~mm} . \times 1.5 \mathrm{~mm}$. subglabris; corollae tubo extus sparse piloso $S \cdot 5 \mathrm{~mm}$. longo, lobis apice barbatis latiusculis ; otario tha ro-arachnoideo.

In the forest, in soil which is inundated during the rainy season, Lower Congo, Cabra 93! Hb. Brux.

A very distinet speeies, recalling the Ameriean S. colombiana. The eritical features are the braets, the long calyx-lobes, and the short corolla.

37. S. Schumanniana Büttner, in Verh. Bot. Ver. Brand. xxxi. 76 (1890).

Frutex ramulis appresse pilosis glabrescentibus ; foliis ellipticis utrinque breviter attenuatis supra sparse scabrello-hirtis infra sparsissime nisi in renis dense sericeo-strigosis, petiolo demum ad ca. $1.5 \mathrm{em}$. longo, stiputis late ovatis $7 \mathrm{~mm} . \times 6 \mathrm{~mm}$. reflexis; inflorescentia laxiorum inter compactas, pedunculo $2-3 \mathrm{~mm}$. v. subsessili, bracteis paucis inconspicuis lanceolatis $3-4 \mathrm{~mm}$. longis nec involucrantibus; calycis lobis lineari-lanceolatis ad 7-8 mm. longis; corollae albae extus sparsiuscule sericeo-strigosae tubo $18 \mathrm{~mm}$., lobis lanceolatis $5 \mathrm{~mm}$. longis (PI. XII).

Congo: between Lukeola and Bolobo, Büttner 447 ! Hb. Berol. Dewévre 145! 287 ! Eala, Pynacrt 1268! Leopoldville, Gillet 2572 ! Hb. Brux. I refer two Gold Coast Plants-Thompson 46 ! and Burton! Hb. Kew.- with some hesitation to this speeies; they may be distinet, but the material is inadequate for certain identifieation. A plant from the Ivory Coast, Dabou, Chevalicr 17232 ! is probably also referable to the same speeies.

The speeies is remarliable for the length-over $2 \mathrm{~cm}$.- of the flowers.

\section{S. angolensis Wernham.}

Frutex volubilis caule 6-10-pedali juniore appresse pubescente $v$ strigilloso inox glabrescente; foliis ellipticis breviter acuminatis 7-8 cm. $\times 3-3 \cdot 5 \mathrm{~cm}$, petiolo saepius brevi nonnunquam ad $1 \mathrm{~cm}$. longo, supra sparsissime patule hirtis infra nisi in venis densiuscule strigosis glabratis, stipulis orato-lanceolatis $7-8 \mathrm{~mm} \times 3 \cdot 5 \mathrm{~mm}$. 
mox reflexis deciduis ; inflorescentia laxiuscula subcapitata pedunculo vix $1 \mathrm{~cm}$. necnon manifesto, pedicellis accrescentibus in fructu ad 4-5 mm., bracteis paucis ovalibus primo subinvolucrantibus ca. 5-8 $\mathrm{mm}$. $\times 3-4 \mathrm{~mm}$.; calycis lobis lineari-oblongis fere ad $1 \mathrm{~cm}$. accrescentibus deinde patulis; corollae albae tubo extus glaberrimo tubulari $8-9 \mathrm{~mm}$. nec insuper ampliato, lobis patentibus $3 \mathrm{~mm}$. longis, extus dense sericeo-barbatis (PI. XII).

Angola: Cazengo, Gossweiler $601 !$ Golungo Alto, Welwitsch 4744 ! 4745 ! To 3000 ft. Hbb. Mus. Brit., Kew., Berol., De Cand., Mus. Paris.

39. S. Vogelii Benth., in Hook. Niger Fl. 398 (1849), including var. villosior.

Frutex scandens caule gracili sparsiuscule breviter villoso glabrescente ; foliis ellipticis $7-10 \mathrm{~cm} . \times 3-5 \mathrm{~cm}$., acuminatis basi saepius rotundatis, petiolo $5-10 \mathrm{~mm}$., utrinque hirto-pilosis pilis saepius patentibus, venis secundariis paucis distantibus, stipulis oblongis ad rotundis ad $1 \mathrm{~cm} . \times 7 \mathrm{~mm}$., mox reflexis; inflorescentia laxiorum inter compactas subsessili v. nonnunquam pedicello manifesto ad $3 \mathrm{~cm}$. nonnunquam $5 \mathrm{~cm}$. longo, bracteis paucis lanceolato-ovatis nonnunquam subinvolucrantibus; calycis lobis linearibus 11-12 mm. demum superantibus; corollae tubo 10-15 mm. longo extus glabrato, lobis suberectis $2 \mathrm{~mm}$. longis extus nec conspicue barbatis.

Sierra Leone: Don! Vogel 87 ! Barter ! Scott Elliot 3871 ! 4175 ! Afzelius! Dinklage 2509! Reade (Hb. Kew.)! French Guinea: Farmar 234! Kaloum, Maclaud 358! Diaguissa, high plateaux, Chevalier 12687! Hbb. Mus. Brit., Kew., Berol., De Cand., Deless., Mus. Paris.

S. Vogelii leads directly to Capitatæ (see p. 16, and Fig. 3), being nearly related to $S$. tchapensis and its allies.

\section{Sectio ii. Sessıles Wernliam.}

Bracteis saepius inconspicuis nec involucrantibus, inflorescentia sessili saltem nec capitulo pedunculato involucrato, floribus dense fasciculatis sessilibus v. subsessilibus rarissime solitariis.

40. S. speciosissima K. Schum., in Engl. Bot. Jahrb. xxxiii. 338 (1903).

Frutex, foliis oblongis ca. $10 \mathrm{~cm} . \times 4 \mathrm{~cm}$., utrinque pilis inspersis, stipulis e basi late triangulari abrupte acuminatis et subulatis $1 \mathrm{~cm}$. longis; floribus solitariis subsessilibus; calycis lobis lineari-subfiliformibus ca. $3 \mathrm{~cm}$. longis; corollae clavatotubulosae tomentosae tubo ca. $8 \cdot 5 \mathrm{~cm}$., lobis subulatis ca. $1.5 \mathrm{~cm}$.; antheris $1 \cdot 5 \mathrm{~cm}$. longis (PI. XII).

Cameroons: Kebo, $650 \mathrm{ft}$., Conrau 247! Hb. Berol.

Unique for the genus in the size of its flowers. 
41. S. speciosa K. Schum., in Engl. Bot. Jahrb. xxiii. 429 (1896).

Frutex scandens ramulis dense patente rufo-hirsutis; foliis $10-15 \mathrm{~cm} . \times 5-7 \mathrm{~cm}$. ; floribus in fasciculis sessilibus lateralibus densis dispositis, rel nonnunquam subsolitariis; calycis lobis Iongiuscule subulatis ; corolla rufo-sericeo-hispida $3-4 \mathrm{~cm}$. longa (Pl. XII).

Togo: Büttner 263! S. Nigeria: Oban, Talbot 1040 ! Cameroons : Conva 218! Bipinde, Zenker 1816! Victoria, Winkler 27 ! Barombi, Preuss 150 ! 325! Abonando, Rudatis $57 ! 800 \mathrm{ft}$. Hbb. Mus. Brit., Kew., Berol., Boiss., Deless., Mus. Paris.

A striking species, on account of its large flowers, with long subulate calyx-segments, bristling, likc the young branches, with reddish hairs.

\section{S. medusula K. Schum., MS.}

Herba repens caule radicante vix nisi basi lignoso, insuper pilis rutis patentibus ciense induto; folizs ad ca. $10 \mathrm{em} . \times 5 \cdot 5 \mathrm{~cm}$. plerumque obovatis nec acuminatis, valde inaequalibus, majoribus basi obliquis, subtus diseoloribus lanugine albido minuto tamen denso indutis, stipulis integris; inflorescentiis dense fasciculatis sessilibus; calycis lobis subsetaceis, patente hirsutis ad $5 \mathrm{~mm}$. longis; corolla $8-2 \mathrm{~mm}$. longr (Pl. V I, 1-3).

Cameroons: Bipinde, Zenker 2095! 4414! Hbb. Mus. Brit., Kew., Berol., Deless., Holin., Mus. Paris.

40-50 cm. in length. Leaves hairy on both sides, silvery bclow: Stipules membrnnous, triangular or lanceolate, up to about $14 \mathrm{~mm}$. $\times 4 \mathrm{~mm}$. Remarlsable for its lowly habit and its anisophylly.

43. S. acuminata Baker, in Journ. Linn. Soc. xxv. 320 (1890)

Suffrutex erectus 7 -pedalis, ramulis dense hirsutis vix arachnoideis; foliis subcoriaceis lanceolatis ad ovatis longiuscule acuminatis, nonnunquam inaequalibus, majoibus ca. $12 \mathrm{~cm} . \times$ $4 \mathrm{~cm}$. minimis $5 \mathrm{~cm}$. $\times 2 \mathrm{~cm}$, basi obliquis, utrinque molliter hirsutis nec arachnoideis subtus discoloribus velutinis, stipulis in setis pluribus fimbriatis; floribus sessilibus fasciculatis; calycis lobis angustissimis subsetaceis ad ea. $8 \mathrm{~mm}$. longis; corolla vix $1.5 \mathrm{em}$. longa ; stylo nonnunquam ad $6-7 \mathrm{~mm}$. e flore exserto (Pl. VII, 1-3).

Madagascar: Baron 5736! 6238! Petit-Thouars! Norontsanga, Hildcbrandt 3024! Nossi-Bé, Joivin 2064 (in part) *! Richard 211! I. Stc. Marie, Boivin 2064 (in part)! Richard 636! Hbb. Mus. Brit., Kcw., Jerol., Mus. Prris.

\footnotetext{
* See next apecies.
} 
The inequality of the leaves is not so marked as in S. diversifolia from this $S$. acuminata is distinguished by its hirsute, not arachnoid, indumentum, and the numerous long stipular setae.

\section{S. angustifolia Boivin MS.}

Frutex subsarmentosus ramulis arachnoideis mox glabrescentibus vix nisi novellis pubescentibus; folits lineari-oblongis ca. $11 \mathrm{~cm} . \times 1.5 \mathrm{~cm}$.; membranaceis, basi saepe rotundatis saepius nec conspicue acuminatis, acutis, supra asperulo-pubescentibus subtus in maturitate sparsiuscule arachnoideis, nec valde inaequalibus, petiolo ad $5 \mathrm{~mm}$. longo, stipulis parvis ovatis setosofimbriatis; floribus subsessilibus in axillis fasciculatis; calycis lobis setaceis patente pilosis ad $1 \mathrm{~cm}$. demum attingentibus ; corolla anguste infundibulari, extus sericea, tota ca. $6 \cdot 5 \mathrm{~mm}$. longa, lobis lanceolatis $2 \cdot 5 \mathrm{~mm}$.

Madagascar : Nossi Bé, forest above Panandara, Boivin 2064 (in part)! Hbb. Boiss., Mus. Paris.

The nearest ally is $S$. aeuminata, from which this differs in its long nurrow leaves and corolla barely half the size.

45. S. diversifolia Per's. Syn. i. 203 (1805). Don, Gen. Syst. iii. 539. Drake del Castillo, Hist. Nat. Pl. Madag. vi. t. 448. Schoenkfeldia diversifolia Spreng. Syst. i. 765.

Frutex erectus ca. 7-8-pedalis ramulis arachnoideis; foliis superioribus plerumque valde inaequalibus inferioribus subaequalibus, majoribus ovato-lanceolatis acuminatis basi obliquis, supra primo arachnoideis demum glabris, subtus lanuginosovelutinis discoloribus ad $15 \mathrm{~cm} . \times 5.5 \mathrm{~cm}$., minoribus ad ca. $2 \mathrm{~cm} . \times 1 \mathrm{~cm}$., stipulis late ovatis subpectinatim fimbriatis; floribus flavis in cymis arcte sessilibus fasciculatis; calycis lobis angustissimis subsetaceis ca. $8 \mathrm{~mm}$. longis; corollae extus aureosericeae tubo $1-1 \cdot 7 \mathrm{~cm}$. longo, lobis ad $6 \mathrm{~mm} . \times 2 \mathrm{~mm}$.; stylo e corollae tubo ad $7 \mathrm{~mm}$. exserto, stigmate conspicue 5-lohato (Pl. XII).

Mauritius? Petit.Thouars! (v. infra). Madagascar: Baron! Bernard! Breon 27! Chapelier! Forbes! Gerrard 24! Humblot 69 ! Lastelle! Lyall! Petit-Thouars! Poivre! Thompson! Janala Country, Baron 298 ! Forest of Didy, Catat 1722 ! Forest near Ankeramadinika, Seott Elliot 1772 ! Betanimena, Hilsenberg! I. Ste. Marie, Boivin 1767! Bernier 271! Forbes! Riehard 34! Maroa, forests in the interior, bay of Antongil, Mocquerys 162 ! 264! Hbb. Mus. Brit., Kew., Cantab., Berol., Boiss., De Cand., Deless., Mus. Paris.

The locality given for the type by Persoon is Mauritius, and he records it as collected there by Petit-Thouars ("Dom. Aubert"). I can find, however, no specimen from Mauritius in any of the European herbaria which I have examined-all being from Madagascar; and Don's description and Drake's excellent figure leave little doubt 
regarding the identity of the species. It would appear not unliliely, therefore, that the locality Mauritius was given by Persoon in crror.

The species is at once recognisable by its erect habit, the cottony indumentum and light-coloured felt on the under-side of the rather broad leaves, and the great inequality of the latter. This makes them appcar alternately arranged; but in Richard's plant cited abore (n. 34 in Hb. Mus. Paris) the leares at the lower nodes tend to equality; hence it may be that the anisophylly obtains only in the upper branches.

According to Bernier (n. 271, in Hb. Mus. Paris) the common name is Voa Scira (or Sena? sce next species), and a decoction of the leares is useful in fercr-cases.

46. S. seua Wernham. Seua (nom. rulg.) Flacourt, Hist. Gr. Isle de Madagascar, 137, 140 (1661).

Frutex ramulis arachnoideis; foliis valde inaequalibus majoribus and $13 \mathrm{~cm} . \times 4 \mathrm{~cm}$, milloribus $1.5 \mathrm{~cm} . \times 6 \mathrm{~mm}$, supra nisi in velia centrali densiuscule sparsim hirto-pubescentibus, subtus dense flavo-lanuginosis valde discoloribus, petiolo brevissimo; stipulis membranaceis ovatis ca. $8 \mathrm{~mm}$. $\times 7 \mathrm{~mm}$, in laciniis 5-8 lanceolatis divisis, dorso et margine puberulis; floribus in fasciculis arcte sessilibus, bracteis lanceolatis subinvolucrantibus ; calycis lacimiis lanceolatis complanatis $4-5 \mathrm{~mm} . \times 1.5 \mathrm{~mm}$., intus subglabris extus dense flavo-arachnoideis.

Madagascar: Flacourt 98 ! 126 ! ex Hb. Taillant, in Hb. Mus. Paris. Clcarly related to $S$. diversifolia and $S$. acuminata, but at onec distinguishable by the calyx-lobes. The latter', together with the anisophyllous character, may connect this species with the west African S'. Mildlbraedii.

The leaves are said to possess astringent properties, and to be uscful for fomenting contusions, etc.

47. S. cinerea Aubl. Pl. Guian. 193, t. 75 (1775). Schwent: felda cinerea Sw. Fl. Ind. Occ. i. 452, t. 10. Schuenlifeldia cineren Sw. loc. cit.; Willd. Sp. Pl. i. 982.

Frutex scandens ramulis arachnoideis; foliis plerumque ellipticis ca. $11 \mathrm{~cm} . \times 5 \cdot 5 \mathrm{~cm}$., supra primo arachnoideis demum glabris infra discoloribus lanugine dilute flavo denso induto ; inflorescentia lanuginosa sessili, floribus arcte compactis, bracteis ovalibus ad ca. $9 \mathrm{~mm} . \times 8 \mathrm{~mm}$. conspicuis subinvolucrantibus; calycis lobis lanceolatis vel oblongis nec angustissimus car. $7 \mathrm{~mm}$. longis; corollue albae densiuscule extus patente hirsutae tubo 12-15 mm. longo, lobis lanceolatis ad $6 \mathrm{~mm}$. longis; bacer rosea v. obscure purpurea ca. $8 \mathrm{~mm}$. $\times 7 \mathrm{~mm}$., a calycis tubo $4 \mathrm{~mm}$. lobis $1 \mathrm{~cm}$. longis persistente coronata (Pl. XII).

Guiana: Aublet! Hb. Jussicu! Iseblond! Patris! Martin 82 ! French Guima: Leprieur 101! 102 ! Hb. Moricand! Perrottet! Poitcau! Cayenne, Jelsti: Kunth! Leguillon: Martin $95 !$ Perottet! 
D. Richard! Rothery 231! Karouany, Sagot 309! Koarou, L. Richard ! Porto Pico: Ledru 349! Hbb. Mus. Brit., Kew., Cantab., Berol., Boiss., Brux., De Cand., Deless., Holm., Mus. Paris.

This species has not becn found south of Guiana, in contrast with the somewhat nearly-related $S$. grisea, a native of eastern Brasil, with which it has been confused. S. cinerea is at once distinguishable by the floccose-arachnoid indumentum, which is confined for the most part to the youngest branches in the rougher, villous, S. grisea. The leaves, moreover, soon become smooth and glabrous on the upper surface in the present species. A similar distinction separates S. cana from $S$. brasiliensis, and $S$. diversifolia from $S$. acuminata. A further and important distinction lies in the conspicuous and involucrate bracts of the present specics; the bracts are much smaller and less conspicuous in S. grisea.

\section{S. mollissima Benth. MIS.}

Frutex scandens ramulis dense patente villosis, caule basi repente radicante; foliis ad ca. $8 \mathrm{~cm} . \times 3 \cdot 5 \mathrm{~cm}$., supra asperulopubescentibus subtus discoloribus argenteo-arachnoideo-lanuginosis; inflorescentiis bracteatis sessilibus nec multifloris, bracteis exterioribus ovato-lanceolatis ad $14 \mathrm{~mm} . \times 6.5 \mathrm{~mm}$; floribus albis sessilibus confertis; calycis lobis lanceolatis complanatis $5-8 \mathrm{~mm}$. longis; corollae tubo $1 \mathrm{~cm}$. raro excedente, e calyce tandem parum exserto, extus densissime patente sericeo-villoso, lobis lanceolatis ad $4 \mathrm{~mm}$. longis (Pl. XII).

Brasil: about Santarem, Spruce 320! 684! Hbb. Mus. Brit., Kew., Cantab., Berol., Boiss., De Cand., Mus. Paris.

The affinity is with $S$. grisea, but the present species is much more hairy and shaggy with spreading indumentum, the leaves smaller, and the corolla-tube shorter. The wholc plant, too, is apparently much smaller.

\section{S. amazonensis Wernharc.}

Frutex subrepens ad 4-5-ped. adscendens, caule primo densiuscule patente hirsuto demum glabrescente; foliis ca. $12 \mathrm{~cm} . \times$ $5 \mathrm{~cm}$., supra sparsiuscule pilosis subtus discoloribus minute arachnoideo-lanuginosis venis saepius fuscis conspicuis; inflorescentiis sessilibus villosis, bracteis ovatis subinvolucrantibus; floribus confertis, calycis lobis lineari-lanceolatis $7-9 \mathrm{~mm}$. longis ad $2 \mathrm{~mm}$. latis ; corolla alba extus nisi tubi basin versus glabrata dense patente hirsuta, tubo $2 \mathrm{~cm}$. nonnunquam excedente, e calyce $1 \mathrm{~cm}$. exserto, lobis ovato-lanceolatis $4 \mathrm{~mm} . \times 2.5 \mathrm{~mm}$. (Pl. V, 3,4).

Brasil: Amazonas province, Koch 92 ! about Manaos, Ule 5117 ! Guynne-Vaughan 25! Rio Negro, Trail 389! Ega, Pocppig, 2514 ! Venezuela: Pacimo, Spruce, s. n.! Hbb. Kew., Berol., Boiss., Deless.; and a representative shoot of this species, with a fully-opened 
flower, is mixed with S. Jirsuta var. Scllowii under Glaziou 19435a! in hb. Mus. Paris.

Distinct from its allies in the habit, compact subinrolucrate inflorescences, and the large, far exserted corolla.

\section{S. pannosa Wernham.}

Frutex scandens, foliis ad $14 \mathrm{~cm} . \times 5 \mathrm{~cm}$., ellipticis acuminatis, supra hispidulo-pubescentibus subtus arachnoideo-lanuginosis, petiolo $1 \cdot 5-2 \mathrm{~cm}$. longo, stipulis lanceolatis ad triangularibus; inflorescentiis sessilibus paucifioris, floribus confertis sessilibus; calycis lobis longe acuminatis lineari-oblongis ad $12-14 \mathrm{~mm}$. accrescentibus; fructu in diametro ca. $5 \mathrm{~mm}$. rufo-brunneo-piloso a calycis persistentis lobis sinuatis nec rigidis coronato.

Brasil : Prov. Pernambuco, Caxanga, Schenck $4279 !$ Hb. Berol.

Near S. grisca, but distinct in the shape of the calyx-lobes, which curl in the fruiting-stage, and in the long petioles; the character of the indumentum on the underside of the leaves, too, differs in the two species.

51. S. grisea Cham. \& Schlecht., in Limniea iv. 192 (1829). S. eriantha DC. Prod. iv. 439. Schwenlefeldia criantha Dietr. Syn. Pl. i. 793.

Frutex scandens, foliis plerumque ellipticis cn. $12 \mathrm{~cm} . \times 5 \mathrm{~cm}$., supra asperulo-pubescentibus subtus arachnoideo-lanuginosis discoloribus, venis nec fuscis nec conspicuis, petiolo brevissimo raro $1 \mathrm{~cm}$. attingente, stipulis late oratis; inflorescentiis fioribusque confertis sessilibus, bracteis nec conspicuis nec subinvolucrantibus ; calycis lobis lineari-oblongis $6-8 \mathrm{~mm}$. accrescentibus rigidiusculis demum patentibus nec conspicue acuminatis ; corollae albae tubo ad $14 \mathrm{~mm}$., lobis lanceolatis $1-5 \mathrm{~mm}$. extus villosae (Pl. XII).

Brasil: without more precise locality, Blanchet 53 ! Boog! Burchell 774! 1804! 2960! Fra Custodio 98! Dupré! Forrest 38! Glocker 561! 531! Polıl 864! Sello 328! 768! 804! 959! 1076 ! Vauthier 98 ! Ceara, Gardner 1697! Pernambuco, Ridley, Lca of Ramage! Alagoas, Gardncr 1338! Bahia, damp hedges, Blanchet 85 ! 1175 ! 3124 ! \& s. $10 . !$ Guillot 3224 ! \& s. n.! Lhotstiy 558! Hb. Martius! Nadeaud! Salzmann 7 ! \& s. n.! Feira S. Anna, Blanchet! Is. Itaparica, Casarctto 2245! Jacobina, Mordiba. Blanchet! Minas Geracs, S. Hilairc 59! Rio de Janeiro, Bowic \& Cumingham! Burchell 2672! Doellinger! Freyroiss! Frohich! Gandichand. 630! Gay! Glazion 714! S. Hilaire 182 ! Macrae! Hb. Martins ! Raddi! Regnell 92! Schenck: 1697! 3776! Vauthier 41! Weddel 295! Widgren 119! 1047! Corcovado, Gardner 5486! Glazion 8740 (in part)*! Guillomin 129! Micrs 3154! Saldanha 439! Schwacke 4755! Ule 3803! Mandiocea, Ricdct. 623! Paraguay : Fleischer! ex hb. Steudel (Paris). Hbb. Mus. Brit., Cantab., Kew., Berol., Boiss., Brux., De Cand., Dcless., Mus. Paris.

$$
\text { * See Sp. } 54 .
$$


$\Lambda$ hedge and thicket scrambler. All the localities named except Paraguay lie in the extreme east and near the coast, from Rio de Janeiro in the south to Ceara in the north. The localities for S. cinerea (q. v.), on the other hand, all lie in Guiana (with the exception of one record from Porto Rico), on the other side of the Amazon; and S. grisea must undoubtedly be maintained as a distinct species. This and $S$. hirsuta are the only two species found outside the tropics.

\section{S. Burchellii Wernham.}

Frutex scandens ramulis appresse sericeo-pilosis; foliis ellipticis ca. $9 \mathrm{~cm} . \times 3 \cdot 3 \mathrm{~cm}$, utrinque angustatis, supra asperulo-pubescentibus subtus praesertim in venis pilis nonnunquam longiusculis appressis indutis, nec tamen araneosolanuginosis, petiolo saepius brevi ad $1 \mathrm{~cm}$. longo, stipulis ovatis acutis mox reflexis ad ca. $9 \mathrm{~mm} . \times 6 \mathrm{~mm}$. f floribus in capitulis ca. $1.5 \mathrm{~cm} . \times 1.5 \mathrm{~cm}$. paucifloris nonnunquam ad $2 \mathrm{v}$. 1-florem reductis sessilibus confertis, bracteis paucis conspicuis, ellipticis vel oblongis ad $1.2 \mathrm{~cm} . \times 6 \mathrm{~mm}$., extus in vena mediana et maıgine pilosis, subinvolucrantibus basi nonnunquam connatis; calycis lobis linearibus vel lanceolatis $7-11 \mathrm{~mm}$. longis margine pilosis tubo brevissimo; corollne tubo ca. $1.2 \mathrm{~cm}$. longo extus dense et uniforme griseo-sericeo, lobis lanceolatis $3.5 \mathrm{~mm}$. longis acumiatis acutissimis; ovario dense et longe albidosericeo (Pl. XII).

Brasil : between S. Toao and S. Aña, prov. Pará, Burchell 9271 ! Hbb. Kew., Brux., Mus. Paris.

A woll-defined species, the nearest ally being S. amazonensis; from this it is readily distinguished by the indumentum of the leaves and the size and other characters of the corolla.

53. S. velutina Benth., in Hook. Journ. Bot. iii. 219 (1841). S. aspera Aubl. B. velutina K. Schum. in Mart. Fl. Bras. VI. vi. 307. S. velutina Benth. var. oblongifolia Miq. in Linnæa xviii. 615 (1845).

Frutex scandens partibus novellis saepius rufis; foliis ca. $12 \mathrm{~cm} . \times 6 \mathrm{~cm}$., supra asperulo-pubescentibus subtus lanugine molli nonnunquam decidua indutis; floribus candidis in fasciculis axillaribus pilosis nec arachnoideis dense confertis sessilibus; calycis lobis inter breviores linearibus nec setaceis nec multo accrescentibus; corollae extus dense et uniforme patente pilosae tubo ca. $1 \mathrm{~cm}$. longo; bacca ellipsoidea sparsiuscule hirsuta ad $12 \mathrm{~mm} . \times 10 \mathrm{~mm}$. a calycis limbo persistente lobis vix $3 \mathrm{~mm}$. coronato (Pl. XII).

Guiana: Appun 328! Hostmann 40! Jenman 1775! Miquel! Schomburgk 25! Demerara, Hancock 282! Japscooma, Jcnman 1724! 
Maroni, Mellinon 186! Massaroonie, Appun 359! Roraima, Schomburgl: 901! 1333! Trinidad: Fendler 425! 5 miles from Erin, Broadway 2226! Brasil : Pará, Wullschlacgel 253! Hbb. Mus. Brit., Kew., Berol., Brux., De Cand., Deless., Holm., Krug \& Urban, MLus. Paris.

The reddish tinge in the youngest parts and the petioles is eharaeteristie. The Trinidad speeimens reveal the deeiduous eharacter of the felt on the under-surfaee of the lenves.

\section{5t. S. Lindmaniana Wernliam.}

Frutex forsan erectus; foliis supra asperulo-pubescentibus infra lanugino-velutinis discoloribus ca. $7 \mathrm{~cm} . \times 3 \cdot 5 \mathrm{em}$; florilus in cymulis alaribus puberulis paucifloris sessilibus confertis; calycis lobis latiuscule lanceolatis inter breviores nec multo accrescentibus $5 \mathrm{~mm}$. vix attingentibus; corollne extus pilis aseendentibus sparsiuscule hirsutie tubo $T \mathrm{~mm}$. nec excedente (Fl. XIT).

Brasil: Glazion 8740 (in part)! Hb. Holm. Porto d'Estrella, I"arming! Hb. Mus. Paris.

Near S. camporum, but distinct espeeially in the larger size of the ealyx-lobes and the eorolla. The habit is unfortunately doubtful. Two speeies are apparently ineluded under the same number $8740 \mathrm{in}$ Glaziou's colleetion-the one just deseribed, and $S$. grisea $(q, v$. $)$, witll long ealyx-lobes and eorolla-tube.

i.). S. camporum Siprague, in Trans. \& Proc. Bot. Soc. lidin. xxii. 434 (1904).

Irerba suffruticosa erectir 1-2-pedalis ramulis junioribus clense fulro-rillosis nec arachnoideis; foliis ca. $6 \mathrm{~cm} . \times 2 \cdot 5$ cm. supua asperulo-pubescentibus subtus discoloribus albidis minute arachnoideo-lanuginosis; floribus in cymis hirsutis nec lanuginosi. clense confertis sessilibus plurimus; calycis lobis primo vix $3 \mathrm{~mm}$. nec valde accrescentibus lanceolatis; corolla inter minimas tulu 3-4 nm. lobis $2 \mathrm{~mm}$. (PI. IV, う̄-9).

Colombia: Cabuyaro, Rio Meta, in the eampos, Sprague 43 ! Hbb. Mus. Brit., Ken:

One of the few small erect forms among the small-leaved speeies.

\section{S. glomerata Wernlaan.}

Frutex ramulis novellis dense flavo-tomentosis deinde glabrescontibus; foliis late lanceolatis $x$. elliptico-oblongis $10-12 \mathrm{~cm} . x$ 4.5-5) cm. basi sacpius rotundatis breviter acuninatis suprar s]ausim asperulo-puberulis infra dense flavo-lanuginosis, pefolo ad ca. $1.3 \mathrm{~cm}$, stipulis late ovitis membranaceis glabris breviter ancuminatis acutis ad $1.4 \mathrm{~cm} . \times 1 \cdot 2 \mathrm{~cm}$. . flubibus in fascienlis 
globularibus nisi corollis fere glabris multifloris axillaribus sessilibus dense confertis; calycis lobis oblongis subglabris art $3 \mathrm{~mm} . \times 1 \mathrm{~mm}$.; corolla tota vix $7 \mathrm{~mm}$. excedente extus unifor'me dense sericea. Paris.

Colombia: Pilcuan, Barbacoas, 2925 ft., Triana 1756 ! Hb.

Differs from $S$. camporum, its nearest ally, in its habit, which is apparently scandent, and its glabrous, spherical bunches of flowers.

\section{S. brasiliensis Wernham.}

Frutex erectus 3-4-pedalis ramulis arachnoideis ; foliis aequalibus ca. $7 \mathrm{~cm} . \times 4 \mathrm{~cm}$. ellipticis vix acuminatis basi acutis nec obliquis subsessilibus supra asperulo-pubescentibus subtus discoloribus dense albido-arachnoideo-lanuginosis; inflorescentia dense conferta multiflora sessili; calycis extus cano-lanuginosi lobis brevibus triangularibus latiusculis nec acuminatis; corolla candida brevi extus dense incano-lanata lobis ovato-lanceolatis patentibus (Pl. XII).

Brasil: Claussen 560! 568! 609 ! Pohl 148! 957! Richard! Pernambuco, Gardner 2886! Bahia, Blanehet! Minas Geraes. Claussen 271! 673! \& s. n.! S. Hilaire 283! 2281! Regnell 1016! Sehenck: 3379! Weddell 2563 ! Goyaz, Burehell 5136! 6532! 7035 ! 8061! 8383 ! Garlner 3225 ! Caraça, in campo, Glaziou 14912! Lagoa Santa, Warming 105! Andayatuba and Ytú, in campos, Riedel 2065! Faria, Glaziou! Bordo do Campo, dry campos, Riedel, 116! Bolivia: Apolo, Williams, 173 ! 303 ! Mapiri, Rusby, 1905 ! Hbb. Mus. Brit., Kew., Cantab., Berol., Brux., De Cand., Deless., Holm., Mus. Paris.

This species is distinct in several cbaracters, tabulated below, from the Peruvian S. eana (q. v.), with which it has been confused. The latter is a scrambler like $S$. grisea, while $S$. brasiliensis is a short erect shrub growing, like $S$. camporum, in the campos. The present species is figured in Mart. Fl. Brasil., VI. vi. t. 134, under the name S. eana.

\section{S. cana Hook.}

Native of Colombia and Northern Peru.

\section{Twining shrub.}

Leaves arachnoid, otherwise gla. brous, abore, and smooth.

Leaves rounded at the base, often with a short acute portion decurrent into the manifest petiolc.

\section{S. brasitiensis Wernham.}

Native of Bolivia and southern and eastern Brasil.

Short erect shrub.

Leaves more or less densely hairy and hispidulous above, never arachnoid.

Leaves gradually narrowed to the base, subsessile. 
s. cana Hook.

Petioles longer than the inflorescence, sometimes over $2 \mathrm{~cm}$. long.

Stamens inserted half-way down the corolla-tube.

Ovary and stigma 4-merous.

\section{S. brasitiensis Wernham.}

Petioles not exceding the inflorescence.

Stamens inserted in the mouth of the corolla-tube.

Ovary and stigma 5-merous.

58. S. cana Hook. Ic. Pl. t. $24 i$ (1840). S. flavida Krause, in Engl. Bot. Jahrb. xl. 323.

Frutex scandens ramulis arachnoideis; foliis late ovatis ad $13 \mathrm{~cm} . \times 7 \mathrm{~cm}$. vix acuminatis, supra laevibus glabris vel arachnoideis subtus discoloribus dense et minutiuscule cinereoarachnoideo-lanuginosis, basi rotundatis $\mathrm{v}$. subito et breviter in petiolum nommunquam ad $2 \mathrm{~cm}$. longum decurrentibus acuminatoangustatis, stimelis late ovatis obtusiusculis breviter acuminatis $S \mathrm{~mm} . \times 8 \mathrm{~mm}$. ; floribus in fasciculis cano-lanuginosis dense confertis sessilibus, bracteis late ovatis obtusis imbricatis sæpe subinvolucrantibus; calycis lobis latis brevibus triangularibus; corollne extus cano-lanuginosae tubo $4-5 \mathrm{~mm}$. lobis $2-3 \mathrm{~mm}$. orario tetramero (Pl. XII).

Peru: Chicoplaya, etc., Ruiz \& Pavon! Moyobamba, Matthews! Wcberlauer 4568! Cerro de Campaña, Spruce s. n.! Colombia: between Yolombo and Canean, 4000-6000 ft. Lehmann 4004! Hbb. Mus. Brit., Kew., Berol., Boiss.

This seems to be essentially a native of western tropical South America, and I can find no specimen collected in Brasil. The essential points of difference from the Brasilian S. brasitiensis have been indicated under that species. Dr. Krause, following K. Schumann in the Flora Brasiliensis (supra), has treated S. brasiliensis as S. cana and the latter species as new, naming it S. flavida.

\section{S. guianensis Wernham.}

Frutex scandens ramulis arachnoideo-pilosis; foliis $8-9 \mathrm{~cm}$. $\times 3 \cdot 5 \mathrm{~cm}$. ovatis acutis subaequalibus, petiolo brevissimo, supra dense hispidulo-tumentosis infra discoloribus arachnoideolanuginosis; inflorescentiis in axillis stricte confertis sessilibus multifloris lanuginosis bractentis; calycis lobis lanceolatis complanatis in flore ca. $3 \mathrm{~mm}$. mox ad $7-8 \mathrm{~mm}$. accreseentibus setaceo-acuminatis; corolla extus cano-villosae dilute roseae tubo $6 \mathrm{~mm}$. lobis ca. $2 \mathrm{~mm}$. (Pl. XIT).

British Guiana: Mt. Canaupang, Schomlurgks. n.! Hb. Kew.

$\Lambda$ twiner, with pink flowers. 


\section{S. Mildbraedii Wernham.}

Suffrutex repens caule radicante, insuper dense et patento rufo-piloso partibus junioribus saepe purpureis; foliis quemque ad nodum valde inaequalibus, majoribus ad ca. $12 \mathrm{~cm} . \times 6.5 \mathrm{~cm}$. ellipticis basi obliquis petiolatis acutis, supra asperis demum scabrello-pubescentibus subtus discoloribus arachnoideo-lanuginosis, minoribus ca. $9 \mathrm{~mm} . \times 6 \mathrm{~mm}$. late ovatis acutis breviter acuminatis deciduis, stipulis membranaceis ovalibus setaceopectinatis, toto ca. $1 \mathrm{~cm} . \times 5 \mathrm{~mm}$. Floribus albis in fasciculis axillaribus extus lanuginosis dense confertis sessilibus; calycis lobis planis lanceolatis vix $3 \mathrm{~mm}$. longis, apicem rersus saepius purpureo-barbatis; corolla tota vix $4 \mathrm{~mm}$. longa.

Congo: Kimuenza, 1300-1625 ft., Mildbraed 3664! Hb. Berol. Gillet 775 ! Sanda, Vanderyst! Environs of Lemfu, Butaye! Between Deinlo and the Koango, Butaye 1487 ! Hb. Brux.

Distinctive in the purple, dense hairs of the youngest parts, the anisophylly, the stipules, and the low, creeping habit. The last three specimens named above exhibit the progressive tendency to the following variety :-

\section{Var. glabrescens Wernham.}

Ramulis arachnoideis, pilis quoque dilute-flaris adpressis indutis; foliis novellis ipsis supra nisi in rena centrali hirtellis glabris.

Congo: Kisantu, Gillet 3520 ! Hb. Brux.

\section{S. dubia Wernham.}

Frutex v. suffrutex caule dense pilis rufis patentibus induto; foliis membranaceis, supra et margine densiuscule hirsutis subtus praesertim in venis pariter indutis lanugine etiam denso minuto griseis, inaequalibus majoribus obovatis ad $15 \mathrm{~cm} . \times$ $8.5 \mathrm{~cm}$., brevissime acuminatis basi rotundatis obliquis, petiolo dense piloso $2-3 \mathrm{~cm}$. longo, minoribus late ovatis ad suborbicularibus acutis breviter v. vix acuminatis, petiolo nec e caulis pilis apparente, stipulis oblongis extus sericeo-pilosis intus glabris ca. ad medium in laciniis $6-7$ apice setaceis pectinatim divisis, setis ad $2 \cdot 5 \mathrm{~cm}$. v. longioribus $\times 6-7 \mathrm{~mm}$. basi latis.

Congo: Madibi, Sapin! Hb. Brux.

I liave no hesitation in referring this plant to Sabicea; but as no flowers are available, its exact position must remain in some doubt for the present. In its vegetatire organs it approaches S. Mildbraedii; from this it is distinguishable by its much coarser habit, larger and thinner leaves, of characteristic shape, with less strongly-marked anisophylly, and long petioles.

\section{S. Batesii Wernham.}

Frutex sarmentosus caule laevi arachnoideo; foliis rerisimiliter ab abortu alternantibus, ellipticis sieje oblongis basi ralde 
obliquis $20 \mathrm{~cm} . \times 9 \mathrm{~cm}$. in maturitate excedentibus, petiolo $3 \mathrm{~cm}$. longo, supra nisi in rena centrali hirtella glabris subtus araneosolanuginosis, stipulis lanceolatis ad $12-14 \mathrm{~mm} . \times 8-10 \mathrm{~mm}$. interris; floribus in fasciculis extus cano-lanuginosis dense confertis sessilibus; calycis lobis ovatis obtusis $2-3 \mathrm{~mm}$. longis (Pl. VII, 4-7).

Cameroons: Bipinde, Lolodorf, Zenker 4070! Gaboon: bank of stream, Mfoa, 85 mi. E. of Gaboon, Bates $536 !$ Hbb. Mus. Brit., Kerv., Berol., Brux., De Cand., Deless., Mus. Paris.

A "trailing shrub or vine" with flowers and infloreseenees much like those of $S$. brcsitiensis; distinetive in the large size and pseudoalternate arrangement of the leaves. (1899).

63. S. arborea K. Schum., in Engl. Bot. Jahrb. xxviii. 58

Arbor ramulis arachnoideis; foliis lanceolatis attenuatoacuminatis ca. $8 \mathrm{~cm} . \times 3 \mathrm{~cm}$. basi acutis, supra arachnoideis mox glabratis subtus lanuginoso-tomentosis incano-discoloribus; firribus pluribus in fasciculus lanuginosis sessilibus dense confertis ; crlycis dentibus brevibus vix 2 mm. lanceolatis; corollae tubo ca. $5 \mathrm{~mm}$. extus subtomentoso lobis lanceolatis acuminatis 5 mm. ; ovario biloculari. Berol.

Zanzibar coast; Uluguru, 5000-6000 ft., Stuhlmann 8775! Hb.

Peeuliar in its tree-habit and biloeular ovary, The dried speeimens have the typieal appearanee of a Sabicea, reealling $S$. diversifolia, from the neighbouring island of Madagasear, with which it may have some affinity.

64. S. glabrescens Benth., in Kew Journ. Bot. iii. 219 (1841). S. aspera Aubl. $\gamma$. glabrescens K. Schum. in Mart. Fl. Bras. VI. vi. 307 .

Frutex scandens ramulis appresse rufo-hirsutis demum glabratis; foliis ovatis v. ellipticis acuminatis ad $7-10 \mathrm{~cm} . \times$ 3 -4 cm., supra scabrello-pubescentibus vel glabrescentibus infra nisi in venis appresse strigosis sparsissime hirsutis ad glabratis, stipulis late ovatis v. rotundatis saepius mox reflexis; florilus in fasciculis alaribus sessilibus confertis; ealycis lobis ovatis $2 \mathrm{~mm}$. longis nec valde accrescentibus; corollae candidae extus uniforme pilis longis canis patentibus dense indutae tubo $1 \mathrm{~cm} . \mathrm{v}$. longiore lobis lanceolatis 4-5 $\mathrm{mm}$. (Pl. XII).

Guiana: R. Quitaro, Schomburgk 538! Demerara, Parker! Lama Creek, Jenman 3698 ! Hooroobea, Jenman 4711 ! Upper R. Casiquiari. Spruce 3275! Campo de Jannuari, Spruce 1281 ! l'rinidad : Hb. Kru! \& Urban 1074! Brasil: Pará, Schuacke $3452 !$ Hbb. Mus. Brit., Kew., Cantab., Berol., Boiss., Brux., Dc Cand., Deless., Mus. Paris.

Resembles $S$. aspera, but is readily distinguished by the broad stipules and calyx-lobes. A speeimen in the hew herbarium, eollected 
by Jenman (no. 987) on the Essequibo R., may belong here ; but it is described on the label as "shrub or small tree." The leaves are rather larger and relatively broader than in the typical $S$. glabrescens, but the corolla is exactly similar. The material is unfortunately inadequate for treatment as a new species, as may be the case.

65. S. hirsuta H. B. \& K. Nov. Gen. \& Sp. iii. 417 (1818). Schwenkfeldia hirsuta Spreng. Syst. i. 765 (1825). S. villosa Willd. ex Roem. \& Schult. Syst. v. 265 (1819). Paiva vertirillata Vell. Fl. Flum. 104 (1825); iii. t. 16 (1827).

Frutex scandens; foliis elliptico-oblongis acuminatis utrinque plus minus praecipue subtus in venis hirsutis nec tamen lanuginosis, ca. $6-10 \mathrm{~cm} . \times 2 \cdot 5-4 \cdot 5 \mathrm{~cm}$, stipulis late ovatis vel subrotundis reflexis; floribus albis inter minimis in fasciculis alaribus sessilibus confertis; calycis laciniis lineari-lanceolatis planis inter breviores laro ad $4 \mathrm{~mm}$. accrescentibus; corollae tubo extus infla glabrato insuper sparsiuscule strigoso vix $6 \mathrm{~mm}$. longo, lobis anguste triangularibus suberectis minutis; bacca violacen, ad $1 \mathrm{~cm}$. diametro (Pl. XII).

Guatemala: Tïrckheim (J. D. Smith 7749)! Nicaragua: Tate 212! Chontales, Secmann 128! Costa Rica: Tondu\% 9186! 13363! Pittier 1208! 10297! Porto Rico: Bertero! Balbis! Hb. Kunth! Eggers 1065 ! Gundlach 1474! Riedle!! Richard! Sintenis 263! 1819 ! S'tahl 376! Wilson 232! Colombia: Smith 1834 (in part)!* banks of the Orinoco, Humboldt! Humboldt \& Bonpland! Goudot! Trinidad: Broadivay 3337! Lockhart 350! Hb. bot. gard. Trin. 1793! Sieber 327! Guiana: Poiteau! Leprieur! Demerara, Martin 246! Mori. cund 179 (in part)! La Mana, Sagot 880! Cayenne, Jelski! Poiteau! Ecuador: Valle Mindo, Sodiro! Peru: Ruiz \& Pavon! Rivero 379 ! Lima, Ruiz \& Pavon 379! Tarapoto, Spruce 4837! Tatanara, Lechler 2510 ! Tillao, Ruiz! Maynas alto, Poeppig 1821! Brasil : Burchell 3155 ! 3475! Widgren 535! São Paulo, Lindberg 718! Mosen 3416 ! Lagoa Santa, Warming! Rio de Janeiro, Widgren! Santa Catharina, Ule, 182! Blumenau, Miiller, 128! Morretes, Dusén, 4469! Hbb. Mus. Brit., Kew., Cantab., Berol., Boiss., Brux., De Cand., Deless., Holm., Krug \& Urban, Matrit., Mus. Paris.

Beside those quoted above the two following typical specimens are preserved in the Botanic Gardens herbarium at Madrid. Both are from Tropical America, but the precise locality does not appear.

Cavanilles 260 ! giving, as a common name, po-ri. Rodriguez!

The habitat of the specimen described and figured by Vellozo as Paiva verticillata is given as "campis maritimis, praesertim Parochia Campo grande habitat. Mensibus calidis floret." I have not seen it.

\section{Var. a. adpressa Wernham.}

Ramulis foliisque appresse sericeo-pilosis, bacea r'ubra.

Costa Rica: Limon, Kuntze 1995! Punta Arena, Tonduz 6712 ! 9955! Pallama: Hayes! Seemann 1073! Chagres, Ficndler 180 !

\footnotetext{
* Sec species 31 .
} 
Portobello, Billberg! Trinidad, Broadway 3338! St. Thomas Is., Friedrichstal! Brasil: Jurua R., Ule 5118! Maymas, Yurimaguas, in fruticetis, Poeppig! Hbb. Mus. Brit., Kcw., Berol., Boiss., Deless., Holm., Krug \& Urban, Mus. Paris.

\section{Tar. $\beta$. Sellowil Wernham.}

Ramulis sparsim patente pilosis, folior um venis subtus appresse sericeis ; calycis lobis ad $4 \mathrm{~mm}$. accrescentibus latiuseulis marginibus saepe revolutis; corolla minima.

Brasil : Rio de Janeiro, Gaudichand 622 ! et s. n.! S. Hilaire 980 ! Minas Geraes : Tombador, near Diamantina, Glaziou 19435a* (in part)! Bahia: Sello 223! 299! 381! 732! 1072! Illheos: Blanchet 3004! Hbb. Mus. Brit., Kew., Berol., De Cand., Deless., Mus. Paris.

The distribution is the widest of any species (see p. 5). The two varicties, it will be observed, are confined to the western and eastern areas respectively.

'This species is somewliat rariable, notably in the extent of the hairy covcring; in a few cases, again, the calyx-lobes are somewhat accrescent and conspicuously acuminatc aftcr flowering-e.g. Sagot's plant fiom La Mana. The constant features are espccially the corolla -among the smallest in the genus-and the shape of the stipules; both these serve to distinguish $S$. 7irrsuta readily from $S$. aspera, with which it has been confuser.

66. S. aspera Aubl. Pl. Guian. i. 194 t. 76 (1775). Schoenlifeldia aspera Willd. Spec. Pl. i. 982. Nom. Vulg. (Galibi, Guiana) Sabi-Sabi.

Frutex scandens ramulis appresse strigoso-hirsutis; foliis lanceolatis $v$. anguste ovalibus utrinque angustatis, supra asperis breviter hirto-pubescentibus subtus in venis distantibus strigosis aliter sparsissime pilosis vel glabratis nec arachnoideo-lanuginosis, ad $10 \mathrm{~cm} . \times 3 \cdot 5 \mathrm{~cm}$, stipulis lanceolatis acuminatis nec reflexis; Aroribus albis in fasciculis alaribus nonnunquam demum laxescentibus sessilibus confertis; calycis lobis planis linearibus acutis ad $3 \mathrm{~mm}$. longis; corollae extus patente pilosae tubo gracili ca. $1 \mathrm{~cm}$. longo lobis lanceolatis ca. $3 \mathrm{~mm}$. longis (Pl. XIT).

Guiana: Aublet! Patris! Perrottet! Poiteau! Richard! Hb. Moricand! Brasil: Florista de Tijuca, Glazion! Pará, Burchell 9989! 10038! 9346! 9429! Baker 73 ! Hbb. Mus. Brit.. Kew., Bcrol.. Boiss., Brux., Deless., Holm., Mus. Paris.

The secondary veins arc noticeably distant-7-8 pairs at most: and the shape of the stipules is quite unusual for the genus. This species is "transitional" in respect of the inflorescence (sce Introduction); examples showing the tendency to laxity are those collected by Glaziou and by Baker in Brasil. 'The following broad-leaved raricty. also exhibits the same featurc.

* Seo Sp. 49. 
Var. latifolia Wernham.

Rufus, foliis late ovalibus rix $\mathrm{v}$. parum acuminatis ca. 9-12 cm. $\times 5-6 \mathrm{~cm}$., petiolo ad ca. $1 \mathrm{~cm}$.; inflorescentia laxiuscula.

Guiana: Cayenne, Martin 63! Hbb. Mus. Brit., Kew.

\section{S. parva Wernham.}

Suffrutex humiliusculus caule repente radicante, ramis ca. $20-30 \mathrm{~cm}$. altis erectis gracilibus insuper appresse strigosis; foliis inter minores $4-6 \mathrm{~cm} . \times 1 \cdot 5-2 \cdot 5 \mathrm{~cm}$., utrinque acutis lanceolatis $\mathrm{v}$. ovalibus, utrinque sparsissime nisi subtus in venis dense strigoso-hirsutis scabrello-pubescentibus, stipulis angustis oblongo-lanceolatis; floribus pauciusculis in axillis confertis sessilibus; calycis lobis planis lanceolatis acutis vix $1 \mathrm{~mm}$. longis; corollae extus uniforme patente villoso tubo $12-13 \mathrm{~mm}$. longo lobis oblongis $2-3 \mathrm{~mm}$. longis (PI. VI, 4-6).

Brasil : near Caburí, Rio Negro, Trail 391! Hb. Kew.

Remarkable for the habit, and shape of the stipules: the affinity is clearly with $S$. aspera.

\section{S. flagenioides Wernham.}

Frutex ramulis divaricatis apicem versus tomento pátente minutiusculo indutis; foliis inter minores lanceolatis acuminatis ca. $5 \mathrm{~cm} . \times 1.5 \mathrm{~cm}$., supra nitentibus nisi in rena media sparsissime ut infra sparsiuscule puberulis glabris renis secundariis perpaucis valde obscuris, stipulis subulato-setaceis $2-3 \mathrm{~mm}$. longis; floribus paucis in axillis confertis subsessilibus; calycis lobis planis lanceolatis rel anguste triangularibus subacutis, fructu ad $4.5 \mathrm{~mm}$. attingentibus.

Yucatan: Chichankanab, Gaumer 1432! in Field Columbian Museum. Hb. Berol.

\section{S. parviflora K. Schum. MS.}

Frutex scandens ramulis gracilibus rufo-puberulis diraricatis ; foliis inter minores ovatis ad ca. $3 \mathrm{~cm} . \times 1.5 \mathrm{~cm}$., renis obscuris utrinque perpaucis (3-4) basi plerumque rotundatis petiolatis, utrinque nisi subtus in venis sparse strigillosis glabratis, stipulis a basi lato vaginante in setam longiusculam productis; foribus minimis in axillis confertis sessilibus, inflorescentia demum laxescente diametro $1 \mathrm{~cm}$. excedente; calycis lobis ad $2.6 \mathrm{~mm}$. linearibus; bacca glabra ad $6 \mathrm{~mm}$. diam., a calycis limbo lobis ad $3.5 \mathrm{~mm}$. coronata; seminibus angularibus minutissimis tuberculatis.

Congo: Sembo, Allard 343! Kisantu, Gillet 148! Hb. Brux. Angola: Welwitsch 3165! Hb. Berol.

Resembles the prerious species from Yrucatnn; the chief distinction 
lies in the much smaller cymes and flowers in the present spccics, and also the rounded leaf-basc. The inflorescence becoming lax after maturity of the flowers is significant.

\section{Sectio iii. CApl'Ate Wernham.}

Inflorescentia capitata compacta, bracteis involucrantibus, pedunculo manifesto.

\section{S. brevipes Wernham.}

Frutex 5-6 pedalis subscandens caule juniore appresse sericeo-piloso desuper subtomentoso: foliis ovalibus acuminatis basi rotundatis v. subcordatis ad $11-12 \mathrm{~cm} . \times 5 \mathrm{~cm}$., petiolo brevissimo nomunquam ad $1-1 \cdot 5 \mathrm{~cm}$., supra hispidulo-pubescentibus, subtus in venis dense aliter sparsissime sericeo-strigosis nec il'ancoso-lanatis, stipulis pro rata majusculis ovato-lanceolatis $15 \mathrm{~mm} . \times 7 \mathrm{~mm}$, saepius reflexis; floribus albis in capitulo $2-2 \cdot 5 \mathrm{~cm}$. diam. compacto sessilibus, dense confertis, pedunculo l'alo ca. $8 \mathrm{~mm}$. excedente, bracteis ovalibus acutis acuminatis $15 \mathrm{~mm} . \times 8-9 \mathrm{~mm}$. involucrantibus; calycis limbi lobis tubum subaeryantibus $6-7 \mathrm{~mm}$. longis ad $1 \mathrm{~cm}$. v. longioribus accrescentibus, lineari-lanceolatis acuminatis aeutis rigidiusculis sparse pilosis; corollae extus sericeae parum e calyco exsertae tubo ca. $15 \mathrm{~mm}$. longo, lobis ovatis vix $3 \mathrm{~mm}$. longis; bacen rubra subsessili a calycis limbi lobis saepe caudato-acuminatis persistentibus coronata.

Togo: Baumann 256! Kersting 146 ! Bütiner 73 ! 749 ! ]3ukila, E. \& M. Laurent! Hbb. Bcrol., Brux.

A near ally of S. Vogetii in §Laxæ.

71. S. Gilletii De Wild., in Ann. Mus. Congo v. i. 78 (1903).

Frutex scandens ramulis dense pilis patulis rufis $2-3 \mathrm{~mm}$. longis indutis; foliis ellipticis $6-13 \mathrm{~cm} . \times 2-4.5 \mathrm{~cm}$., basi cuneatis, acuminatis acutis, supra sparse subtus praesertim in renis densius pubescentibus nec araneosis, petiolo ad $1-3 \mathrm{~cm}$. clongato, stipulis ovatis acutis reflexis ad $14 \mathrm{~mm} . \times 5-6 \mathrm{~mm}$; Horibus in capitulis confertis, pedunculo $1-1 \cdot 8 \mathrm{~cm}$. dense villoso, bracteis involucrantibus villosis $15 \mathrm{~mm} . \times 10 \mathrm{~mm}$. ovatis acutis : culycis limbo fere ad basin in laciniis lineari-lanceolatis acutis $10 \mathrm{~mm} . \times 1 \mathrm{~mm}$. diviso.

Congo: Kimnenza, Gillet 1911! 2024! Ifnta and Tomkola, E. \& 1. Laurent! Sanda and Kimpako, Vanderyst! Bombaic, Clacssens 173 ! Kasai, Dcmla, in the plain, Supin! Hb. Brux.

72. S. tchapensis Krause, in Engl. Bot. Jahrb. xlviii. 408 (1912).

Frutex scandens lamulis patule pilosis; foliis ellipticis (a. 10-12 cns. $\times$ 4-5 cm. breviter acmminatis, basi angustatis, 
supra scabrello-pilosis subtus nisi in venis saepius patule pilosis slabratis, petiolo nomnunquam ad $3 \cdot 5 \mathrm{~cm}$. elongato, stipulis ovatolanceolatis acutis ca. $1 \mathrm{~cm} . \times 5 \mathrm{~mm}$.; floribus albis in capitulo compacto $2 \cdot 5 \mathrm{~cm}$. diam. sessilibus confertis, pedunculo $6-8 \mathrm{~mm}$., bracteis late ovatis acuminatis ca. $1.5 \mathrm{~cm} . \times 2 \mathrm{~cm}$. involucrantibus; calycis limbi tubo lobos oblongo-lanceolatos $8 \mathrm{~mm}$. longos vix subaequante; corollae e calyce parum exsertae tubo ca. $15 \mathrm{~mm}$. longo, extus nisi infra lobos triangulares acutos erectos 2 mul. longos pariter sericeo glabrato.

Cameroons: Tchape Pass, Ledermann 2655! Lome, Mildbraed 5422 ! Bipinde, Zentier 2968! Hb. Berol.

Var. glabrescens Wernham.

Minus hirsutus pilis appressis, foliorum venis subtus sericeis.

West Africa: Pogge 1041! 1162 ! 1196! Cameroons: Jaunde, Zentier 204! Batanga, Bates 770 ! Hbb. Mus. Brit., Kew., Berol.

The most noticeable feature in this and the two preceding species is the rather large capitulum, with deep, broad, almost orbicular, involucral bracts and very short, rather stout peduncle. The chief point of distinction lies in the shape and dimensions of the involucral bracts.

\section{S. Schaeferi Wernham.}

Frutex ramulis sparse strigosis; foliis ellipticis utrinque angustatis ad ca. $12 \mathrm{~cm} . \times 5 \mathrm{~cm}$, venis secundariis utrinque ca. 15-18, utrinque sparsissime nisi in venis strigillosis hirtopubescentibus v. glabratis nec araneosis, petiolo ad $2 \mathrm{~cm}$., stipulis $7 \mathrm{~mm} . \times 5 \mathrm{~mm}$. ovatis reflexis; floribus roseis in capitulo compacto ca. $2 \mathrm{~cm}$. diam., pedunculo strigilloso ca. $4 \mathrm{~cm}$. dispositis, bracteis ovati-lanceolatis vix $1 \mathrm{~cm} . \times 5 \mathrm{~mm}$. involucrantibus; calycis limbo fere ad basin in laciniis lineari-oblongis $8 \mathrm{~mm}$. longis acutis ciliatis diviso ; ovario extus sparse strigosohirsuto.

Cameroons: Bare, 5800-6500 ft., Schaefer 76 ! Hb. Berol.

74. S. ingrata K. Schum., in Bolet. Soc. Brot. x. 126 (1892).

Frutex ramulis novellis subtomentosis demum glabrescentibus ; foliis ellipticis utrinque breviter acuminatis ca. $11-14 \mathrm{~cm} . \times$ 5) 5-6 cm., supra sparse pilosis subtus discoloribus minute alaneoso-tomentosis, petiolo ad 2.5 cm., stipulis late ovatis vix 1 cin. $\times 7 \mathrm{~mm}$.; floribus in capitulo cumpacto ca. $2 \mathrm{~cm}$. diam. dispositis, bracteis ovalibus $\mathrm{v}$. rotundis ca. $1 \mathrm{~cm} . \times 8 \mathrm{~mm}$. involucrantibus, pedunculo sparsiuscule piloso nec araneosolanato $1-1 \cdot 5 \mathrm{~cm}$. recto rigidiusculo; calycis lobis linearilanceolatis ad $3-4 \mathrm{~mm}$; corollae anguste infundibularis extus pilosae tubo $14 \mathrm{~mm}$. laciniis $3 \mathrm{~mm}$. longis.

St. Thomas Island: Quintas 18! Hb. Berol. 


\section{S. gracilis Wernham.}

Frutex scandens ramulis teneris novellis subtomentosis demum glabrescentibus ; foliis ellipticis ad subrotundis $6-7 \mathrm{~cm} . \times 3-4 \mathrm{cur}$. breviter acuminatis acuto apice basi acutis, supra, nisi in veni centrali hirtis, pilis paucis conspersis, subtus minute araneosolanatis discoloribus, venis saepius conspicuis margine et rena centrali rufo-ciliatis, petiolo ad $3-4 \mathrm{~cm}$.; floribus subsessilibus in capitulis compactis ca. $1 \cdot 5 \mathrm{~cm}$. diam. confertis, bruteis involucrantibus suborbicularibus, pedunculo gracili curvato glabrato $3 \mathrm{~cm}$. v. longiore; calycis lobis lineari-oblongis ad 5-6 mm. acutis: corollae tubo cxtus glabro $8-9 \mathrm{~mm}$., lobis ca. $1 \cdot 5 \mathrm{~nm}$. patentilus ; bacca subsessili nec saltem conspicue pedicellata (Pl. VIII, 1-4).

Cameroons: Batanga, Batcs 398! Hbb. Mus. Brit., De Cand.

The collector makes the note: "Twining vine. Long-and shortstyled flowers on different plants."

76. S. ferruginea Benth., in Hook. Niger Fl. 397 (1849). Cephaëlis fermingen Don, Gen. Syst. iii. 605 (1834).

Frutex scandens ramulis appresse dense rufo-sericeo-pilosis; foliis ellipticis $\mathrm{v}$. oblongis breviter acuminatis $10 \mathrm{~cm} . \times 4 \cdot 5 \mathrm{~cm}$.$15 \mathrm{~cm} . \times 7 \cdot 5 \mathrm{~cm}$. basi saepius rotundatis, supra nisi in vena centrali rufo-strigosis glabris, juventute rufo-hirsutis subtus densissime ferrugineis araneoso-lanuginosis, petiolo appresse piloso saepius brevi $1 \mathrm{~cm}$. raro longiore, stipulis majusculis $12 \mathrm{~mm}$. $\times$ $10 \mathrm{~mm}$. orato-triangularibus acutis extus 3-6-hirsuto-costatis : floribur permultis albis in capitulo compacto $3-4 \mathrm{~cm}$. diam. arcte confertis, pedunculo $5-9 \mathrm{~cm}$. longo dense sericeo-strigoso, bracteis plurinis imbricatis exterioribus late ovatis ad $1.8 \mathrm{~cm} . \times 1.6 \mathrm{~cm}$. longiuscule acuminatis acutis, extus scabrello-pubescentibus margine ciliato; calycis lobis elongatis subfiliformibus demum $1 \mathrm{~cm}$. excedentibus necnon sinuatis, pilis patulis longiusculis villosis; corollae tubo anguste cylindraceo nec insuper ampliato, extus glabrato ca. $13 \mathrm{~mm}$. longo, lobis lanceolatis erectis extus strigillosis rix $2 \mathrm{~mm}$. longis; fructus pedicellis ad $5 \mathrm{~mm}$. $\mathrm{v}$. longioribus accrescentibus (Pl. XII).

Sierra Leone: Don! Purdie! Bayroo River, Mann 862! Liberia, Dinklage 2540! 2563! Hbb. Mus. J3rit., Kew., Berol., Mus. Paris.

Var. lasiocalyx Wernbann. Sabicea lasiocalyx. Staps, in Journ. Lim. Soc. xxxvii. 106 (1905).

Pilis saepius patentibus, inflorescentia villosiore albo-tomentosa.

Liberia: Sinoe Basin, Momrovia, Kakatown, Whyte! Hb. Kcw.

77. S. geophiloides Wernham, in Cat. Talk. Niger. Pl. 41 (1913).

Herba repens hirsuta caule prostrato radicante; follis parvis ovatis $r$. ellipticis $3-3 \cdot 5 \mathrm{~cm} \times 1 \cdot 5-1 \cdot 8 \mathrm{~cm}$. apice acuto rix 
acuminatis basi acutis, utrinque hirsutis nec subtus araneosis, petiolo 5-10 mm., stipulis breviter oblongo-rotundatis $3 \cdot 5 \mathrm{~mm} . \times$ $3 \mathrm{~mm}$. mox reflexis; floribus in capitulo vix $2 \mathrm{~cm}$. diam. confertis, pedunculo gracili sparse piloso $2 \mathrm{~cm}$. v. longiore, biacteis late ovatis extus pilis paucis conspersis margine ciliato $1 \cdot 3 \mathrm{~cm} . \times$ $8 \mathrm{~mm}$.; calycis limbo ad dimidium v. altius in lobis lanceolatooblongis 8-9 mm. longis margine piloso diviso; corollae pro rata latiuscule infundibularis extus glabrae tubo $1.2 \mathrm{~cm}$., lobis oblongis subacutis $3.5 \mathrm{~mm}$. longis, ore $5 \mathrm{~mm}$. lato; bacca hirsutissima sessili $7 \mathrm{~mm}$. longa a calyce persistente coronata (Pl. XII).

S. Nigeria: Oban, Talbot 255! Cameroons: Abonando, Rudatis 44 ! Hbb. Mus. Brit., Kew., Berol.

Notable for its habit; its nearest ally is $S$. yilosa, which is a much larger plant, with larger leaves, inflorescences, and flowers.

78. S. pilosa Hiern, in Fl. Trop. Afr. iii. 76 (1877).

Frutex repens et scandens 10 -pedalis caulibus radicantibus novellis patule pilosis; foliis utrinque sparsiuscule et longiuscule hirsutis nec araneosis $v$. lanatis, inferioribus majusculis saepius obovato-lanceolatis $15-17 \mathrm{~cm} . \times 5-6 \mathrm{~cm}$., basi cuneatis apice breviter et subito acuminatis acu tissimis, petiolo elongato ad $4-5 \mathrm{~cm}$. patule piloso, superioribus minoribus saepe ellipticis, acumine longo nonnunquam angusto $2-3 \mathrm{~cm}$., petiolis multo brevioribus, stipulis ovatis acuminatis acutis $1 \mathrm{~cm}$. $\times 6 \mathrm{~mm}$.; floribus roseis in capitulis paucifloris confertis ad $3-4 \mathrm{~cm}$. diam., capitulis nonnunquam compositis, pedunculo $1 \mathrm{~cm}$. raro excedente, bracteis membranaceis ovitis acuminatis acutissimis involucrantibus ad ca. $1.7 \mathrm{~cm} . \times$ $8 \mathrm{~mm}$., extus pilis paucis conspersis margine ciliato; calycis limbo infra medium in lobis pilosis latiusculis oblongis vel lanceolatis flore $1 \mathrm{~cm} . \times 3 \mathrm{~mm}$. demum ad $2 \mathrm{~cm} . \times 4 \mathrm{~mm}$. accrescentibus diviso; corollae extus strigosae anguste infundibularis tubo $2 \cdot 5-3 \mathrm{~cm}$. lobis triangularibus acutis suberectis vix $2 \cdot 5 \mathrm{~mm}$. longis (Pl. XII).

Cameroons: Barombi, Prenss 15! 278! Batanga, Dinklage 548! Bipinde, Zentier 2053! Kribi, Ledermann 864! Mitdbracd 6201! Corisco Bay: Mann 1866! Gaboon: Munda, Soyaux 220 ! Libreville, Jolly 79 ! Benito, Guizal! Hbb. Mus. Brit., Kew., Berol., Boiss., Brux., Deless., Holm., Mus. Paris.

Jolly describes this as a "très grande liane flemrissant sur les parties qui touchent la terre."

\section{S. Trailii Wernham.}

Suffrutex scandens ramulis novellis patule pilosis demum glabrescentibus; foliis ellipticis inter parva vix ad $5 \mathrm{~cm} . \times 2 \mathrm{~cm}$. sessilibus basi angustatis breviter icuminatis, utrinque hirsutis nec araneosis nec lanatis; floribus albis in capitulis paucifloris ca. $1 \mathrm{~cm}$. diam. sessilibus, pedunculo brevissimo raro $3-4 \mathrm{~mm}$. 
excedente, bracteis $2-3$ oratis ca. $1 \mathrm{~cm} . \times 5 \mathrm{~mm}$. basi comnatis involucrantibus; calycis limbi infundibularis tubo ca. $7 \mathrm{~mm}$. longo lobis lanceolatis ciilatis flore ca. $3 \mathrm{~mm}$. demum ad 5-6 mm. accrescentibus ; corollac tubo gracili extus patule villoso $10-12 \mathrm{~mm}$. longo infra lobos laneeolatos extus barbatos $3 \mathrm{~mm}$. longos ampliato (Pl. XII).

T. Anrazon: Obydos, Trail 390 ! Hb. Kew. and s. n.! ex hb. Glaziou in Hb. Mus. Paris.

This and the two following species are interesting as being the only American representatives of the section Capitatac (sce Introduction, and Fig. 3).

\section{S. mattogrossensis Wernlam.}

Frutex scandens ramulis sparsiuseule strigosis deinde pubescentibus glabrescentibus; foliis ellipticis utrinque angustatis ca. $9 \mathrm{~cm} . \times 3 \cdot 5 \mathrm{~cm}$., supra asperulo-pubeseentibus subtus pratsertim in venis strigosis nec araneosis v. lanatis, petiolo ad $1 \mathrm{~cm}$. tenero, stipulis parvis ovatis ad $7 \mathrm{~mm} . \times 7 \mathrm{~mm}$. reflexis; florilus albis in capitulo confertis paucifloro ca. $2 \mathrm{~cm}$. diam., pedunculo vix $1 \mathrm{~cm}$. dense strigoso, bracteis ovatis ea. $12 \mathrm{~mm}$. $\times$ $6 \mathrm{~mm}$. imbricatis involucrantibus; calycis limbo fere ad basin in lobis foliosis oratis ad $9 \mathrm{~mm} . \times 5 \mathrm{~mm}$. extus sparsissime pilosis liviso; corolla parum e calyce exserta, tubo ad $1.5 \mathrm{~cm}$. desuper glabrato insuper sparse strigoso, lobis lineari-oblongis $4-5 \mathrm{~mm}$. longis; orario strigoso (PI. VITI, 5-8).

Matto Grosso: Santa Cruz, Spencer Moorc 785 ! Hbb. MIns. Rrit., licw., Berol.

\section{S. Trianae Wernham.}

Frutex scandens ramulis arachnoideo-lanatis demum glabris; foliis ellipticis utrinque angustatis $8-9 \mathrm{~cm} . \times 3 \cdot 5-4 \mathrm{~cm}$., supria nisi in vena centrali sparse strigosis glabris, novellis sparse arachnoideis nec aliter hirsutis, subtus densius pariter indutis, petioln araneoso-lanato $5 \mathrm{~mm}$. longo, stipulis parvis ca. $6 \mathrm{~mm}$. $\times 4 \mathrm{~mm}$. deflexis; floribus in capitulis paucifloris ca. $1 \cdot 5 \mathrm{~cm}$. diam. ecnfertis, lrecteis paucis involucrantibus, pedunculo brevi ad $3-4 \mathrm{~mm}$. ar aneoso-lanato; calycis lobis ovalibus acntis ad $9 \mathrm{~mm}$. $\times 4 \mathrm{~mm}$.; avario lanuginoso.

\section{Colombia: Trianu 717 ! Hb. Mus. Brit.}

Nearly allied to the preceding Brasilian species, which it resembles in the inflorescence and calyx-lobes, but readily distinguishable by the light-coloured felt on the branchlets, the under side of the leaves. peduncles, and orary, as well as by the glabrous or scantily cottony. upper leaf-surface. The inflorescence tends to become lax after. maturity; the peduncle is shorter. the petioles shorter and stouter. than in S. mallogrossensis. 


\subsection{S. rufa Wermham.}

Frutex scandens parvus ramulis patule purpureo-pilosis demum glabrescentibus; foliis ellipticis basi angustatis breviter acuminatis 8-10 cm. $\times 4-5 \mathrm{~cm}$., supra sparsiuscule hirtis subtus lanugine araneoso minuto deciduo indutis, margine ciliato venis utrinque ad 18 conspicuis patule villosis, petiolo rufo-villoso ad $1.7 \mathrm{~cm}$. longo, stipulis late ovatis v. subrotundis ca. $5 \mathrm{~mm} . \times$ $7 \mathrm{~mm}$. mox reflexis; floribus albis in capitulis parvis compactis 4-6-floris confertis diametro vix $1 \cdot 5 \mathrm{~cm}$., bracteis ovatis extus villosis ca. $1 \mathrm{~cm} . \times 1 \mathrm{~cm}$. involucrantibus, pedunculo pilis purpureis deflexis induto $1 \cdot 5-2 \cdot 5 \mathrm{~cm}$. longo ; calycis limbo obsoleto; corollae tubo infra medium leniter angustato insuper ampliore, extus glabrato $8-9 \mathrm{~mm}$. longo, lobis extus sericeis lineari-lanceolatis vix $1 \cdot 5 \mathrm{~mm}$. longis; ovario extus densissime cano-sericeo; bacea globosa dense villosa $8 \mathrm{~mm}$. diam (Pl. XII).

Cameroons : Bipinde, Zenker 1818! Ebolowa, Mildbraed 5619! Batanga, Dinklage 1063 ! 1153 ! 1211 ! 1363 ! Gaboon: Soyaux 16 ! Hbb. Mus. Brit., Kew., Berol., Boiss., Deless., Mus. Paris.

83. S. ealyeina Benth., in Hook. Niger Fl. 399 (1849). Nom. vulg. (Gaboon): Phaoloi (Jolly).

Suffrutex scandens ad 15-pedalis ramulis sericeis rarissime sparse patente pilosis mox glabrescentibus; foliis plerumque ellipticis $\mathrm{v}$. oblongis brevissime acuminatis basi saepius cordatis $8 \mathrm{~cm} . \times 4 \mathrm{~cm}$. ad $15 \mathrm{~cm} . \times 8 \mathrm{~cm}$, utrinque nisi in venis praecipue subtus strigosis fere glabris, petiolo strigoso-pubescente ad $3-4 \mathrm{~cm}$. longo, stipulis ovatis acutissimis vix $1 \mathrm{~cm} . \times 6-7 \mathrm{~mm}$.; floribus albis v. flavido-albis in capitulis longepedunculatis confertis multifloris, pedunculo gracillimo ca. $8 \mathrm{~cm}$. noununquam ad $12 \mathrm{~cm}$. vel longiore laevi glabro, bracteis albidis insuper siepius viridiusculis basi purpureo-maculatis, involucrantibus paucis exterioribus late ovatis v. suborbicularibus saepe cordatis basi connatis, extus glabratis $15-17 \mathrm{~mm} . \times 15-17 \mathrm{~mm}$. vel majoribus ; culycis limbo fere ad basin in lobis foliosis ovatis $\mathrm{v}$. oblongis majoribus ca. $8 \mathrm{~mm} . \times 3 \mathrm{~mm}$. ad $1.5 \mathrm{~cm} . \times 5 \mathrm{~mm}$. accrescentibus diviso; corollae extus saepius glaberrimae tubo ca. $1.5 \mathrm{~cm}$, lobis parvis $1.5 \mathrm{~mm}$. longis late triangularibus; fructus pedicellis ad 5-6 mm. v. longioribus accrescentibus (Pl. XII).

Ivory Coast : Chevalier 17722! Gold Coast : Farmar 379! Brown 404 ! Abdulke, Krause! Ashanti, Cummins 148! Togo-land, Baumann 163! Lagos: Dodd 420! Rovuland 55! Moloney 25! Nigeria : Benin, Palisot de Beauvois! Eppah, Barter 3282! Between Gogo and Anton, Elliott 77 ! Iro, Foster 185 ! Oji River, etc., Kitson! Oban, Talbot 114a! Eket, along the rivers, Talbot s. n. ! Old Calabar, Robb! Fernando Po: Mann 53! Barter! Vogel 35! Buchholz! Shoropion, Cross River, Holland 31 ! 137! Cameroons : Zenter d Staudt 303 ! Zenker 1140! 2997! 3877! 4568! Nachtigall! Tessmann S83! 
Broun 2! Pieuss 70! 159! 1151! 1334! Ledermann 1014! 6066 Biisgen 239! Mildbrace 5573! Rudatis 21! Dinklage 707! Bates 70 ! 224! Johnston! Wintiler 39! Gaboon : Libreville, Klaine 2684 ! 2805! 2847! 2918! Jolly 29! Congo: Mildbraed 2787! 2983! Pynaert 211! Ketabi, Lecomte E 110 ! \& s. n.! Benito, Guizal! Kaquinga, $E$. of M. Laurent! Dinna, Lescrauvaet 364! Malo, Brinecl! Hbb. Mus. Brit., Kew., Berol., Boiss., Brux., Deless., Mus. Paris.

A very distinct species, with its long glabrous peduncles and heads of Howers with large foliaceous calyx-lobes. The pediccls oftcn elongate in the fruiting stage, and the involucral bracts become reflexed-an obrious device for seed-dispersal. Zenker \& Staudt's no. 303, with dense spreadin hairs on the branchlets, giving an almost woolly appearance, may be a distinct variety. The Howers may apparently be white, yellowish-white, or even violet-coloured (Leconte).

Var. hirsutiflora Wernliam.

Planta floribus extus plus minus sparse strigillosis; corollae lobis angustioribus.

Togo: Von Doering 237! Lagos: Millen 63! 163 ! Rowland! MeucGregor 35! Punch 63! Yoruba: Millson! Between Ibadau and Abcokuta, Schlechter 13024! Hbb. Mus. Brit., Kew., Berol., Brux., De Cand., Deless., Mus. Paris.

\section{S. Barteri Wernham.}

Suffirutex repens 1-2-pedalis caule subtus radicante novello sparse patule piloso; foliis parvis ad $6 \mathrm{~cm} . \times 2.5 \mathrm{~cm}$. ellipticis basi sapius rotundatis $\mathrm{v}$. cordatis supra scabrello-hirtis $\mathrm{v}$. glabratis subtus praesertim in venis strigosis nec araneosis, petiolo ad $1 \mathrm{~cm}$., stipulis ovatis $4-5 \mathrm{~mm} . \times 4-5 \mathrm{~mm}$. mox reflexis; floribus roseis in capitulis $2-3 \mathrm{~cm}$. diam. paucifloris dispositis, pedunculo glabro 3-4 cm., bracteis 3-4 late ovatis ca. $12 \mathrm{~mm}$. $\times 9 \mathrm{~mm}$. glabris saepe rotundatis involucrantibus; calycis limbo in laciniis late lanceolatis ad $11 \mathrm{mn} . \times 3 \mathrm{~mm}$. glabris acutis fere ad basin diviso; corollae extus glaberrimae tubo anguste infundibulari ca. $1 \mathrm{~cm}$. longo, lobis brevibus lato ovatis; ovario glaberrimo; fructus pedicellis ad $6 \mathrm{~mm}$. (Pl. XI, 5-7).

Nigeria: Barter 1248! Old Calabar, Holland 118! Hb. Kew.

The only specics with any manifest resemblance to the preceding one. It is altogether a smaller plant, being a lowly undershrub.

\section{S. composita Wernham.}

Frutex scandens parva ramulis appressissime strigosis; folii. oralibus ad ca. $9 \mathrm{~cm} . \times 5 \mathrm{~cm}$. basi rotundatis acutis vix acuminatis, supra sparse asperulo-hirtis subtus linugrine armeoso dilute brunneo indutis, venis secundariis conspicuis, petiolo ad $2 \mathrm{~cm}$., stipulis oblongo-ovalibus fere ad $1 \mathrm{~cm} . \times 7 \mathrm{~mm}$. acutis nec reflexis: floribus albis in capitulo ad $I \cdot T$ diam. compacto multi- 
floco confertis, bracteis saepius 4 decussatis oblongis infra connatis involucium tubo $1 \mathrm{~cm}$. alto lobis $1 \mathrm{~cm} . \times 8 \mathrm{~mm}$. obtusis formantibus extus lanatis, pedunculo dense strigoso ad $3.5 \mathrm{~cm}$.; calycis lobis lanceolatis acutis $5 \mathrm{~mm}$. longis; corollac extus dense sericeae tubo $9 \mathrm{~mm}$. lobis angustis acutis vix $1.5 \mathrm{~mm}$. longis.

Cameroons: Mibo, $3900 \mathrm{ft}$, Ledermann 1515! Hb. Bero!.

\section{S6. S. brunnea Welnham.}

Frutex scandens ramulis dense patule rufo-pilosis; foliis ellipticis ad ca. $11-12 \mathrm{~cm} . \times 5 \cdot 5-6 \mathrm{~cm}$. utrinque acutis, supra asper'ulo-pilosis subtus lanugine araneoso indutis discoloribus, petiolo vix ad $1.5 \mathrm{~cm}$., stipulis ovatis $5-7 \mathrm{~mm} . \times 4-6 \mathrm{~mm}$. nec leflexis; floribus albis in capitulo $8-10 \mathrm{~mm}$. diam. compacto paucifloro confertis, bracteis 2 exterioribus ovato-oblongis obtusis connatis, involucrum oblongum cujus tubo $5 \mathrm{~mm}$. lobis $5-8 \mathrm{~mm}$. toto $1 \mathrm{~cm}$. altitudine excedente formantibus, pedunculo $1 \cdot 5$ $2 \cdot 5 \mathrm{~cm}$. dense rufo-hirsuto; calycis lobis subulato-lanceolatis 2-3 mm.; corollae extus insuper sericeae infra glabrae tubo angusto 7-8 $\mathrm{mm}$. lobis lanceolatis brevissimis; ovario extus densissime sericeo-strigoso ; bacca alba succo sanguineo (Pl. XIT).

S. Nigeria: Okuni and Ekom, Holland 262! Cameroons: Rudatis 71! Winkler 1027! Hbb. Mus. Brit.. Kew., Berol.

87. S. capitellata Benth., in Hook. Niger. Fl. 398 (1849). S. trichochlamys K. Schum., in Engl. Bot. Jahrb. xxxiii. 338.

Frutex sarmentosus ramulis strigosis in siccitate rufescens; foliis ovalibus $10-15 \mathrm{~cm} . \times 4 \cdot 5-8 \cdot 5 \mathrm{~cm}$., supra scabrello-pubescentibus subtus araneoso-lanuginosis incanis, venis secundariis utrinque $12-15$, petiolo elongato nonnunquam longissimo, stipulis rotundatis reflexis ca. $5 \mathrm{~mm} . \times 8 \mathrm{~mm}$. ; floribus albidis in capitulis 6-10-floris ca. $1 \mathrm{~cm}$. diam. compactis confertis, pedunculo obsoleto ral'o fere ad $1 \mathrm{~cm}$. attingente densissime strigoso-sericeo, bracteis involucrantibus saepius 4 decussatis late ovatis acuminatis acutis ca. $6 \mathrm{~mm} . \times 4 \mathrm{~mm}$. extus lanatis basi connatis; calycis lobis linearibus ad 3-4 mm.; corolla extus superne sparse sericea, tubo ad $1 \mathrm{cin}$. insuper infundibulari ore $3-4 \mathrm{~mm}$. lato, lobis ovatis patentibus $2-3 \mathrm{~nm}$. longis; fructus albidi pedicellis nec conspicuis (PI. XIT).

Fernando Po: Mann 41! Vogel 88! Mildbraed 6851! Cameroons : Bates 78! 78a! Buchholz! Büsgen 484! Dinklage 561! 788! 1062 ! 1362! Ledermann 714! Mann 2198! Zenker 1141! Gaboon: Libreville, Klaine 766 ! 812 ! 1532!2869! Congo: Basin of R. Nsele, Butaye 1459! Hbb. Mus. Brit., Kew., Berol., Boiss., Brux., Mus. Paris.

The type is Mann 41. The specimen in the Paris herbariun 
has leares exceeding $15 \mathrm{~cm} . \times 8 \cdot 5 \mathrm{~cm}$.-broadly elliptie oblong-with petiole over $11 \mathrm{~cm}$. long. The flowers are said to be violet in Klaine's Gaboon plants.

Val. insularis Wernham.

Planta minus hirsuta, nec ramulis nec inflorescentia alachnoideis ; foliis supra fere glabris ; calycis lobis $4.5 \mathrm{~mm}$. fructu ad $5 \mathrm{~mm}$. lineari-oblongis accrescentibus ; corolla minore, tubo $6 \mathrm{~mm}$. lobis $1.5 \mathrm{~mm}$. longis.

St. Thomas Is. : Chevalier 14622! Hb. Mus. Paris.

S8. S. Talbotii Wernham, in Cat. Talb. Niger. Pl. 43 (1913).

Frutex samentosus ramulis strigosis; foliis ellipticis $8-9 \mathrm{~cm} . \times$ $3 \cdot 5-4$ cm. supra scabrellis subtus sparsissime nisi in venis densiuscule strigosis nec araneosis nec lanatis, petiolo ad. 1 em., stipulis inter minores; floribus in capitulis compactis confertis ca. $1 \mathrm{~cm}$. diam., pedunculo brevissimo, bracteis paucis ovalibus involucrantibus basi connatis; calycis limbo in laciniis linearibus aiviso vix $3 \mathrm{~mm}$. longis, corolla extus insuper strigosa ca. $1 \mathrm{~cm}$. longa lobis brevibus angustis, ovario densissime cano-sericeohilsuto (Pl. XIT).

S. Nigeria: Oban, Talbot 2032! Hb. Mus. Brit.

Very near the preceding species, but easily separated by the absence of felt on the leaves; also the involueral bracts are bearded on the inside in this species, not glabrous as in S. capitellata; and the heads tend to be aggregated at the ends of the shoots in S. Talbotii.

\section{S. fulva Wernham.}

Frutex parva scandens ramulis dense fulvo-tomentosis ; foliis ellipticis ad $9-10 \mathrm{~cm} . \times 5-5 \cdot 5 \mathrm{~cm}$., supra sparse scabrello-hirtis subtus in venis dense fulvo-sericeo-strigosis aliter sparse puberulis nec araneosis, petiolo ad $1-2 \mathrm{~cm}$., stipulis parvis ovatis ca. $4-5 \mathrm{~nm}$. basi $5 \mathrm{~mm}$. latis; floribus albis in capitulis compactis confertis diam. vix $1 \mathrm{~cm}$., pedunculo obsoleto v. brevissimo, bracteis paucis involucrantibus extus densissime fulvo-sericeis imbricatis, exterioribus 2 altitudinis usque ad $3 \mathrm{~mm}$. connatis; calycis limbo in laciniis lanceolatis acutis 4 1nm. longis ca. ad medium diviso; rorollae anguste infundibularis tubo extus infra glabro insuper dense sericeo-strigoso $7 \mathrm{~mm}$, lobis ereetis lanceolato-oblongis $3 \cdot 5 \mathrm{~mm}$. longis extus densissime sericeis.

Cameroons : Lomi, Mildbracd 5424! IIb. Berol.

Differs from the preceding species especially in the shape of the corolla.

90. S. Johnstonii K. Schum. MS.

Frutex ramulis plus ninus appresse rufo-pilosis; foliis ellipticis ca. $8.5 \mathrm{~cm} . \times 4 \mathrm{~cm}$. breviter acuminatis acutis basi saepius 
angustatis, supra in venis praesertim in centrali strigillosis $\mathrm{v}$. puberulis aliter nisi in juventute hirtellis glabratis, subtus in venis strigosis conspicuis, secundariis utrinque 15-18, aliter lanugine alaneoso candido minuto indutis discoloribus, petiolo ad ca. $8 \mathrm{~mm}$., stipulis late ovalibus $4-5 \mathrm{~mm} . \times 5-6 \mathrm{~mm}$. reflexis; floribus in capitulis compactis $2-3 \mathrm{~cm}$. diam. multifloris confertis, pedunculo arachnoideo vel dense appresse rufo-piloso $5-6 \mathrm{~cm}$., bracteis paucis ovatis acuminatis ca. $1 \mathrm{~cm} . \times 6 \mathrm{~mm}$. involucrantibus, extus saepe araneoso-lanatis intus glabris demum reflexis, pedicellis 2-3 mm. gracilibus fructu ad 5-7 $\mathrm{mm}$. v. longiore elongatis; ralycis limbo infra medium in laciniis lanceolatis acutis flore ad 4 inm. fructu ad $7 \mathrm{~mm}$. diviso; corollae extus glabrae tubo insuper infundibulari $7 \mathrm{~mm}$. longo ore $2-3 \mathrm{~mm}$. lato, lobis latis vix $2 \mathrm{~mm}$. longis deflexis ; baccis glabrescentibus ad $5 \mathrm{~mm}$. diam. in capitulis globosis ad $3 \mathrm{~cm}$. diam. dispositis, pedicellis srepius conspicuis (Pl. XII).

Nigeria: Cross River Expedition, Johnston! Old Calabar, Holiand 95 ! Eket, along the rivers, Talbot! Hbb. Mus. Brit., Kew., Berol.

91. S. pedicellata Wernham, in Cat. Talb. Niger. Pl. 42 (1913).

Frutex scandens ramulis arachnoideis canis demum glabrescentibus; foliis ovato-ellipticis longiuscule acuminatis obtusiusculis, $8-10 \mathrm{~cm} . \times 3 \cdot 5-5 \mathrm{~cm}$., supra in vena ipsa centrali glaberrimis juventute sparse arachnoideis nec aliter pilosis subtus dense arachnoideo-lanatis discoloribus, venis secundariis utrinque ca. 12, petiolo ad $1 \mathrm{~cm}$., stipulis a basi $3-4 \mathrm{~mm}$. lato $4-5 \mathrm{~mm}$. longis demum reflexis ; floribus in capitulis ca. 1.5 diam. confertis multifloris, pedunculo $1 \cdot 5-3 \mathrm{~cm}$. araneoso, bracteis involucrantibus paucis ovatis $\mathrm{v}$. suborbicularibus nec acuminatis vix imbricatis subuniseriatis, $5.5 \mathrm{~mm} . \times 5 \mathrm{~mm}$., extus araneosis intus glabris fuscis demum reflexis; calycis lobis brevissimis oblongis mox valde deflexis $2 \mathrm{~mm}$. $\times 1 \mathrm{~mm}$; corolla a basi leniter ampliata extus nisi loborum deltoideor'um brevium basin versus puberula glabra, ad $8 \mathrm{~mm}$. longa, ore $2.5 \mathrm{~mm}$. lata; ovario subgloboso extus arachnoideo; fructus pedicellis gracillimis ad $7 \mathrm{~mm}$. longis (Pl. XII, showing fruit, $a$, and flower, $b$ ).

South Nigeria: Oban, Talbot 1367 ! 2033 !

Very distinct in its uniseriate, early reflexed involucre, and the very short, relatively broad calyx-lobes, which are tooth-like in the flower, soon becoming strongly deflexed and adpressed to the ovarywall. Apart from the whitish felt the plant is practically glabrous.

\section{S. lanuginosa Wernham.}

Frutex scandens ramulis incano-arachnoideis demum glabrescentibus; foliis ovalibus vix acuminatis obtusis ad $12 \mathrm{~cm} . \times 6 \mathrm{~cm}$, 
supra ipsa in rena centrali glaberrimis, juventute sparse arachnoideis subtus dense arachnoidco-lanatis pallide discoloribus, venis secundariis utrinque $10-14$, petiolo $1 \cdot 5-2 \cdot 5 \mathrm{~cm}$, stipulis late oblongo-ovatis ca. $4 \mathrm{~mm} . \times 5 \mathrm{~mm}$. reflexis ; floribus in capitulis ca. $1.5 \mathrm{~cm}$. diam. confertis multifloris, pedunculo $3-4 \mathrm{~cm}$. araneoso, capitulis nonnunquam 2-3 in cyma 2-3-chotoma confertis, bructeis involucrantibus paucis saepius ovatis ca. $5 \mathrm{~mm}$. $\times 4 \mathrm{~mm}$., extus araneosis intus glabris fuscis; calycis lobis oblongo-lanceolatis ad 3-4 mm., demmm patentibus nec valde reflexis; corollac extus arachnoideo-rglabratae tubo $6-\bar{i} \mathrm{~mm}$. insuper parum ampliato, lobis ovatis $1.5 \mathrm{~mm} . \times 2 \mathrm{~mm}$. deflexis ; fructu manifestc pedicellato (Pl. IX, 1-3).

Lagos: Miller 50! 68 ! 70! Hbb. Kew., Berol.

\section{S. brachiata Wernham.}

Frutex scandens ramulis cclerrime glabrescentibus, florentibus $8-12 \mathrm{~cm}$. longis divaricatis; foliis ellipticis vix acuminatis ad ca. $7 \mathrm{~cm} . \times 4 \mathrm{~cm}$., supra in rena centrali glaberrimis juventute sparse arachnoideis subtus griseis arancoso-lanuginosis, venis secundariis paucis utrinque 6-8, petiolo vix ad $1 \mathrm{~cm}$; floritus. albis in capitulis confertis $1 \cdot 5-2 \mathrm{~cm}$. diam. multifloris, iracteis involucrantibus paucis ovalibus ca. $5 \mathrm{~mm} . \times 4 \mathrm{~mm}$, pedunculo araneoso ad $1 \cdot 5-1 \cdot 8 \mathrm{~cm}$; calycis lobis lanceolatis ca. $3 \mathrm{~mm}$. longis nec reflexis; corolla extus dense sericeo-pilosa, insuper parum ampliata $8-9 \mathrm{~mm}$. longa, lobis angustis $1.5 \mathrm{~mm}$. longis, fructu subsessili.

Cameroons: Tibati, $2900 \mathrm{ft}$. Ledermann 2450! Hb. Berol.

\section{S. cruciata Welnham.}

Frutex scandens ramulis deusissime rufo-sericeis tarde glabrescentibus; foliis ellipticis ad $12 \mathrm{~cm} . \times 7 \mathrm{~cm}$. brevissime acuminatis acutis, supra ipsis in venis glaberrimis subtus in venis dense sericeis aliter lanugine fusco-rufo minuto densissimo indutis, petiolo ad $1 \mathrm{~cm}$, stipulis ovalibus ad $7 \mathrm{~mm} . \times 6 \mathrm{~mm}$. nec mox reflexis; floribus albo-viridibus in capitulis compactis confertis ca. 2 cul. diam., bracteis involucrantibus paucis, exterioribus 2 ca. $12 \mathrm{~mm} . \times 7 \mathrm{~mm}$. acuminatis connatis, pedunculo ad $5 \mathrm{~cm}$. dense appresse piloso ; calycis laciniis lanccolato-oblongis acutissimis 4-5 mm. longis ciliatis; corollae tubo 9-10 $\mathrm{mm}$. longo basi ventricoso insuper parum ampliato, extus uisi insuper glaberrimo, lobis angustis ca. $1.5 \mathrm{~mm}$. longis suberectis ( $\mathrm{Pl} . \mathrm{X} 11)$.

Cameroons: Tsomi, Mildbracd 5433! Bebni, 'Tessmam 751 ! Hib. J Bcrol. 


\section{S. Duparquetiana H. Baillon MS.}

Frutex ramulis sparsim patente pilosis; foliis saepe obovatis $8 \mathrm{~cm} . \times 4.5 \mathrm{~cm}$. raro excedentibus, supra nisi in vena centrali subtus nisi in venis strigillosis glaberrimis, reticulo tertiario subtus valde conspicuo; floribus in capitulis ca. $1.5 \mathrm{~cm}$. diam. paucifloris compactis, pedunculo validiusculo $5 \mathrm{~mm}$. longo, bracteis paucis involucrantibus exterioribus 2 connatis sparse strigosis; ralycis sparsiuscule strigillosi tubo ca. $3 \mathrm{~mm}$., lobis fere ad $1 \mathrm{~cm}$. infra medium erectis insuper curvato-patentibus; corollae tubo latiusculo insuper leniter ampliato extus strigoso infra glabrato, 8-9 $\mathrm{mm}$. longo, ore $3-4 \mathrm{~mm}$. lato, lobis validis lanceolatis $5 \mathrm{~mm} . \times 2 \mathrm{~mm}$.

Gaboon: Duparquet! Hb. Mus. Paris.

This and the next species are distinguished by their relatively smooth and subglabrous organs in comparison with the typical members of the genus; and by the compact, few-flowered capitula with very definite involucres of connate bracts.

\section{S. Robbii Wernham.}

Frutex 12-15-pedalis ramulis strigosis tardiuscule glabrescentibus ; foliis plerumque ellipticis ad 9-11 cm. $\times 4 \mathrm{~cm}$., obtuso apice, basi acutis, supra praecipue in venis asperulo-pubescentibus subtus pariter nisi in venis strigosis indutis nec araneosis nec lanatis, petiolo $15-14 \mathrm{~mm}$., stipulis ovatis ca. $4 \mathrm{~mm} . \times 4 \mathrm{~mm}$. $\mathrm{mox}$ leciduis: floribus in capitulis ca. $1 \cdot 5 \mathrm{~cm}$. diam. paucifloris compactis nonnunquam 2-3 in cyma 2-3-chotoma confertis, pedunculo validiusculo saepe leniter curvato $2-2 \cdot 5 \mathrm{~cm}$. sparsiuscule et inconspicue strigoso, bracteis paucis involucrantibus 2 exterioribus connatis ovatis concavis parte libero ca. $8 \mathrm{~mm}$. connato ad 2-3 mm., extus sparse strigillosis; calycis tubo $2.5 \mathrm{~mm}$. lobis 5-6 mun. longis oblongis extus strigillosis ad 9-10 mm. accrescentibus; corollae tubo latiusculo insuper leniter ampliato extus in dimidio inferiore glabro insuper lineatim strigoso, $8-9 \mathrm{~mm}$. longo, ore $3-4 \mathrm{~mm}$. lato, lobis patentibus crassiusculis lanceolatis ad $4 \mathrm{~mm}$. $\times 1.5 \mathrm{~mm}$. (Pl. X, 1, 2).

Old Calabar: Robb! Hb. Mus. Brit. Gaboon: Libreville, Klaine 771 ! 2531! Sea-shore, Thollon 14! Njobe, Ogoouc, Thollon 60! Hb. Mus. Paris.

Nearly related to the preceding species, from which it differs in the indumentum of the shoot, the larger and differently-shaped leaves, and the length of the peduncle. The infloresccnce is typically an umbel of 3-4 heads, with a common and partial involucres.

97. S. trigemina K. Schum., in Engl. Bot. Jahrb. xxviii. 59 (1899). Nom. vulg. (Pahouin) : Oudvilioui.

Frutex scandens ramulis sericeo-strigosis tardiuscule glabrescentibus; foliis ovatis v. oblongis in ramulis florentibus ad 
$8-11 \mathrm{~cm} . \times 3-5 \cdot 5 \mathrm{~cm}$., venis secundariis utrinque ca. 9, in sterilis ad $14 \mathrm{~cm} . \times 9 \mathrm{~cm}$. renis utrinque 20 , utrinque nisi in venis supra sparsissime subtus densius strigosis glabris, petiolo $1.5 \mathrm{~cm}$ $2 \cdot 5 \mathrm{~cm}$, stipulis ovatis acutis $3-14 \mathrm{~mm} . \times 4-15 \mathrm{~nm}$. reflexis; floribus albis in capitulis 3-6-floris dispositis $1.5-2 \mathrm{~cm}$. diam., pedunculo 5-8 mm. validiusculo, Uracteis involucrantibus ovatis acutis basi vix connatis extus strigillosis; calycis limbo tubuloso extus sericeo tubo $3-4 \mathrm{~mm}$. lobis $2 \cdot 5-3 \cdot 5 \mathrm{~mm}$. oblongis obtusis; corolla infundibulari extus sericea tubo $9-10 \mathrm{~mm}$. ore $4 \mathrm{~mm}$. lato lobis patentibus lanceolatis $4 \mathrm{~mm}$. longis ; bacca rubra (Pl. XII).

Cameroons: Bipinde, $1300 \mathrm{ft}$., Zenker 1821! Gaboon: Libreville, Klaine! Jolly 126! Hbb. Mus. Brit., Kew., Berol., Boiss., Mus. Maris.

\section{S. Laurentii De Wild. Miss. Laur. 276 (1906).}

Frutex scandens ramulis appresse pilosis demum glabrescentibus; foliis ad $6-7 \mathrm{~cm} . \times 3-3 \cdot 5 \mathrm{~cm}$. ellipticis basi acutis breviter acuminatis, utrinque nisi sparsissime in renis strigosis glabratis, petiolo ad ca. $12 \mathrm{~mm}$., stipulis rotundo-ovatis $5 \mathrm{~mm} . \times$ $5 \mathrm{~mm}$. ; floribus albis in capitulis parvis $7-9 \mathrm{~mm}$. diam. pauciflor is confertis, peduneulo strigilloso $1-2 \cdot 5 \mathrm{~cm}$. longo, bracteis involucrantibus ovatis decussato-imbricatis basi subliberis extus gli1bratis; calycis limbo in laciniis $2 \mathrm{~mm}$. obtusis diviso, tubo 3-4 1nm.; corollae tubo extus glabro 6-7 mu., lobis lanceolati. extus dense suriceis $3 \mathrm{~mm}$. longis; fruct" extus hirsuto, calycis limbo tubuloso ad $4-5 \mathrm{~mm}$. longo persistente coronato.

Cameroons: Tessmann 654! Lomi, Mildbraed 5416! Ebolowa, Mildbraed 5621! Congo: Eala, M. Laurent 902! Pynaert 510! 1228 ! 1710 ! Bomdaka-Nbolé, Flamigni 73 ! Basoko forest, Clacssens 648 ! 742 ! Kasai, Pogge 979 ! Hbb. Berol., Brux.

Var. velutina De Wild., loc. cit.

Planta villosior praesertion fructu.

Congo: Lulonga, F. \& M. Laurent! Likimu, Malchair 328! Boanga, espersen! Hb. Brux.

De Wildeman (loc. cit.) recognises a seeond variety, Pynacrlii. with the eharaeter's of glabrous ealyx-lobes longer than the tube of the limb. I am inclined, however, to refer the plants on whieh he bases this variety, named below, to the type-speeies:-

Congo: New Anvers, Pynaert 12 ! Eala, Pynaert 579! Hb. 13rux.

The calyx in this speeies and its allies displays a notieeable tendeney to glabrousness, and the relative size of the lobes and linb, is largely a matter of stage of development; I have found the latter elaracter frequently misleading in dealing with this genus.

99. S. Dinklagei K. Schum., in Engl. Bot. xxiii. 428 (1897).

Frutex glaber scandens al 14-pedalis ramulis nisi natu minimis sparse strigillosis glaberrimis : folizs olliptico-oblongis 
breviuscule obtuse acuminatis basi acutis, fere glabris, $3-7 \mathrm{~cm} . \times$ $1-3 \mathrm{~cm}$., venis secundariis utrinque $6-7$, petiolo brevissimo vix ad $3 \mathrm{~mm}$. f floribus in capitulis paucifloris parvis vix $1 \mathrm{~cm}$. diam. confertis, pedunculo $1 \cdot 5-2 \mathrm{~cm}$. glabro, involucri bracteis extimis suborbicularibus obtusis glabris intimis spathulatis multo minoribus; calycis tubo $3-4 \mathrm{~mm}$. lobis vix $2 \mathrm{~mm}$. obtusis ; corolla extus superne strigosa tubo $4 \mathrm{~mm}$. lobis $1 \cdot 5 \mathrm{~mm}$. longis; bacca grisea succo nigricante (Pl. XII).

Cameroons: Batanga, Dinfilage 1124! 1284! Congo: Bangala, $1000 \mathrm{ft} .$, Hens $139 !$ Hbb. Kew., Berol., Brux., Deless.

Similar to the preceding species, but readily distinguished by the almost glabrous branchlets and peduncles, and by the smaller and differently-shaped corolla. (1901).

100. S. Dewevrei De Wild., in Ann. Mus. Congo III. i. 112

Frutex scandens ramulis pilis longis patulis sparsim indutis tarde glabrescentibus; foliis ellipticis utrinque acuminatis $15 \mathrm{~cm} . \times$ 6-7 cm., apice obtuso margine longe piloso, aliter mox glabrescentibus, venis secundariis utrinque 8 paullo prominentibus, stipulis suborbicularibus glabris fere $1.5 \mathrm{~cm}$. longis ; floribus albis sessilibus in glomerulis, pedunculo $1 \cdot 5-3 \mathrm{~cm}$. longe primo hirsuto demum glabro confertis, bracteis involucrantibus suborbicularibus $2-2.5 \mathrm{~cm} . \times 2-2.5 \mathrm{~cm}$. basi connatis intus ad basin pilosis; calycis limbo tubuloso $12-15 \mathrm{~mm} . \times 5-6 \mathrm{~mm}$., lobis 5 brevibus 2-3 mm. $\times 2-3 \mathrm{~mm}$. margine ciliato, intus nisi ad basin piloso glabro; corolla tubulosa exserta apice strigoso tubo gracili extus basin versus glabro $15-18 \mathrm{~mm} . \times 1 \mathrm{~mm}$, lobis 5 ovatis acutis ca. $2 \mathrm{~mm} . \times 1 \mathrm{~mm}$. extus longe pilosis; ovario subglabro.

Congo: Waboundou, Dewèvre 1143! Eala, M. Laurent 1597! Bombé, coffee-plantations, Claessens 711 ! Hb. Brux.

Var. a. latifolia De Wild., Miss. Laur. 276 (1906).

Planta foliis multo majoribus $18-22.5 \mathrm{~cm} . \times 8-12 \mathrm{~cm}$. saepius basi cordatis late ovalibus.

Congo: Isangi, Ifuta, E. \& M. Laurent! Hb. Brux.

Var. $\beta$. glabra Wernham.

Ad 6-pedalis scandens, ramulis petiolisque glabris; foliis minoribus; pedunculis $5-11 \mathrm{~mm}$. glaberrimis; floribus roseis (Pl. IX, 4, 5).

Congo: Irumu, 3250-3575 ft., Mildbracd 2823 ! Hb. Berol.

101. S. gigantea Wernham. Nom. vulg. (Kasai): Mokessé.

Arbor magna ramulis glabris; foliis oblongis ad $15 \mathrm{~cm} . \times 6$ $\mathrm{cm}$. saepius breviter acuminatis, utrinque nisi subtus in renis et margine pilis paucis conspersis strigillosis glaberrimis, petiolo ad 
$1 \cdot 5 \mathrm{~cm}$. subglabro vel supra strigoso, stipulis glabris ovatis vix acutis ca. $1.7 \mathrm{~cm} . \times 1.6 \mathrm{~cm}$; floribns in capitulis subsessilibus pedunculo slaberrimo confertis ca. 6-1loro ca. $2 \cdot 5 \mathrm{~cm}$. diam., bractcis $2-3$ suborbicularibus ca. $1.5 \mathrm{~cm} . \times 2 \mathrm{~cm}$. glaberrimis connatis, involucrum cupularem intus basi dense sericeo-pilosum formantibus; calyce tubuloso ca. $15 \mathrm{~mm}$. $\times 5 \mathrm{~mm}$. dentibus oblongis obtusis $3 \cdot 5 \mathrm{~mm}$. demum ad $6 \mathrm{~mm}$. longis, basi $2.5 \mathrm{~mm}$. latis.

Kasai : Sapin! Hb. Brux.

"Grand arbre des forêts." The mounted specimens bear a close resemblance to the preceding species; the chief distinction lies in the trec-habit, in the almost complete glabrousness, the size and shape of the stipules and of the calyx-lobes.

The strueture of the cupular involuere in this and the preceding species illustrates the transition to the genus Slipularia. 'The bracts are often commate in the inflorescences of Capitatae, but never to such a degree as in these two species, which appear to be considerably isolated from the rest.

\section{Sectio iv. Frombunde: Wernham.}

Frutices scandentes; infloreserefice cymosa composita demum paniculata diflusa laxa foribunda, cymulorum brerteis conspicuis.

102. S. segregata Hieru, in Fl. Trop. Afr. iii. 77 (1STT). S. Henningsiana Biittn., in Verh. Jot. Ver. Brand. xxxi. 79.

Frutex scmdens 15-pedalis, ramulis dense appresse pilosis demum glabrescentibus; foliis ovatis saepe longiuscule acuminatis acutis and ca. $11 \mathrm{~cm} . \times 5.5 \mathrm{~cm}$. basi rotundatis, supra asperulopulescentibus subtus nisi in venis utrinque 12-16 appresse pilosis glabrescentibus, petiolo $1-2 \mathrm{~cm}$. , stipulis ovatis acutis ra. $7 \mathrm{~mm}$. $\times 9 \mathrm{~mm}$. extus glabratis; foribus griseis vix $5 \mathrm{~mm}$. longis breviter pedicellatis in paniculis cymosis compositis demum valde diffusis et laxis dispositis, cymulorum lracteis conspicuis anguste ovalibus $\mathrm{v}$. oblongis nec involucrantibus; calycis lobis ad $4 \mathrm{~mm}$. sublinearibus; corollae extus puberulae tubo ca. $3 \mathrm{~mm}$. lobis ovatis; ovario biloculari; bacca sparsissime puberula a calycis persistentis lobis $5-6 \mathrm{~mm} . \times 1-1 \cdot 5$ coronata, paniculo fructescente ca. $9 \mathrm{~cm} . \times 5 \mathrm{~cm}$. metiente.

Cameroons: Bipinde, Zentier! Gaboon: Klaine 100! 123!377! liver Muni, Mann 1766! between Gaboon and Sibange, Bültnor 437 ! 443! Dinklage 591! French Congo: Ogooué, N'Djolc, Thollon 117 ! Hbb. Kew., Berol., Mius. Paris.

The nearest ally to the next species; but the inflorescences are much less extensive and the bracts less conspicuous.

103. S. floribunda K. Schum., in Kugl. Bot. Jahrl. xxiii. 429 (1897).

Frutex seandens ramulis appresse pilosis deinde glabratis ; folits ovatis v. oblongis breviuscule acuminat is acutis, $\mathbf{L}-17 \mathrm{~cm}$. 
$\times 5 \cdot 5-8 \cdot 5 \mathrm{~cm}$, basi rotundatis $\mathrm{v}$. subcordatis, utrinque nisi subtus in venis supra in rena media appresse pilosis glabris, venis secundariis utrinque 20 v. pluribus, petiolo $1-2 \cdot 5 \mathrm{~cm}$, stipulis ovatis acuminatis extus puberulis; floribus albo-viridibus in cynulorum paniculis ad $30 \mathrm{~cm}$. longis diffusis laxis floribundis folia longe superantibus sessilibus, pedunculo valido $12 \mathrm{~cm}$. v. longiore, cymulorum bracteis foliaceis conspicuis suborbicularibus ad ca. $12 \mathrm{~mm} . \times 9 \mathrm{~mm}$. ; calycis lobis obtusis glabris subfoliaceis ca. $2 \mathrm{~mm}$. longis; corolla $5 \mathrm{~mm}$. longa extus nisi in loborum apice glabra cylindracea tubo $1.5 \mathrm{~mm}$. lato; fructu albido.

S. Nigeria: Oban, Talbot 228! Cameroons: Ledermann 918! Mitibraed 5122! 5141! Rudatis 52! Staudt 1! 159! Tessmann 716! Zentier 1820! 3232! Congo: Djuma valley, Gentil! Kimuenza, Gillet 1749! Hbb. Mus. Brit., Kew., Berol., Boiss., Brux., De Cand., Deless., Holm., Mus. Paris.

\section{Var. paueinervis Wernham.}

Foliorum venis secundariis distantibus utrinque 10-12 planta omnino glabrior (Pl. X, 3-5).

Cameroons : Batanga, Dintilage 743 ! 1391 ! Hb. Berol.

A very distinct species, at once recognizable by the large, very diffuse, panicle of small cymes, with conspicuous orbicular bracts, and small tubular glabrous flowers.

\section{SPeCIES AFFinitatis DUBIж.}

\section{S. bracteolata Wernham.}

Frutex ramulis dense rufo-pubescentibus validiusculis tarde glabrescentibus; foliis ellipticis ad ca. $10.5 \mathrm{~cm} . \times 4.5 \mathrm{~cm}$, utrinque angustatis, utrinque nisi in venis pubescentibus v. strigillosis glabris, venis secundariis utrinque ca. 10, petiolo ca. $11 \mathrm{~mm}$, stipulis ovatis ca. $3-4 \mathrm{~mm} . \times 3 \mathrm{~mm}$. nisi parte inferiore brevissimo persistente caducis nec reflexis; flovibus saepius in axillis solitariis, pedicello $2-3 \mathrm{~mm}$. cujus in apice bracteolis saepius 2 ovatis acuminatis $1-1 \cdot 5 \mathrm{~mm}$. longis patentibus; calycis extus dense appresse-pubescentis lobis oblongolanceolatis ca. $4 \mathrm{~mm}$. subacutis tubo $2 \mathrm{~mm}$. longo; corollae extus dense appresse subsericeo-pubescentis tubo latiusculo, in alabastro cylindrico ad $14 \mathrm{~mm}$. metiente.

French Guinea: Labé, Chevalier 12390! Hbb. Kew., Berol., De Cand., Deless., Nus. Paris.

None of the specimens which I have examined bears an open corolla; and the extreme reduction of the inflorescence further masks the affinity of the species-though it is undoubtedly, I think, referable to Sabicer. 
105. S. verticillata Wernham.

Frutex ramulis dense et longiuscule flavo-viride pubescentibus ; foliis in verticillis saepius 3 -natis, coriaceis, obovatis v. ellipticis ca. $7-9 \mathrm{~cm} . \times 3 \cdot 5-4 \cdot 3 \mathrm{~cm}$. breviter subitoque acuminatis subacutis, basin versus rotundatum leniter angustatis, utrinque sparse scabridis, supra dilute viridibus venis conspicuis impressis, subtus griseo-brunneis venis dense puberulis prominentibus, secundariis utrinque ca. 12, petiolo brevissimo validiusculo dense puberulo, stipulis latis breviusculis in setis paucis fere ad basin divisis; floribus in axillis solitariis, pedicello ad ca. $5 \mathrm{~mm}$. hirsuto, bracteolis 2-3 lanceolatis 5 mm. longis; calycis lobis lanceolatis densiuscule hirtis ad $9 \mathrm{~mm}$. longis; corollae tubo gracili, extus sparsiuscule patente piloso, $1 \cdot 7-2 \mathrm{~cm}$. longo apicem versus plus minus subito ampliato, lobis glabris lineari-lanceolatis $9 \mathrm{~mm}$. longis.

North Madagascar: Humblot 213 ! cx Hb. Drake in Hb. Mus. Paris.

Remarkable for the whorled leaves, and the solitary flowers with slender corolla and narrow lobes nearly a centimetre long. The fimbriate stipules suggest affinity with the other Madngasear species; but in view of the verticillate leaves-unique for the genus-and solitary pedicellate flowers, the systematic position of this species must remain in doubt for the present.

\section{Specils axcludend.æ.}

S. aurea Steud. Nom. ed. i. $712=$ Corcocypselum aureum.

S. crinita A. Rich. Mém. Soc. Hist. Nat. Par, v. 228.

A Madagascar plant, which I have examined in the Paris Herbarium. The flowers are in too young a state for certain determination; but I have little hesitation in excluding this plant from the genus Sabicca. The inflorescences are terminal on the shoots and densely clothed with rufous hairs: it may be a new species of Flagenium.

S. edulis Seem. in Hook. Kew Jour'n. iii. (1851) 266.

Collected in Panama on the "Herald" voyage. It is stated to bear an edible fruit, the common name being madroño de comer. In his account of the voyage, however (Bot. Her. 136 (1854)), Secmanı identifies the same fruit with that of Alibertia edulis A. Rich.-which is clearly, therefore, synonymous with Sabicca erlutis.

S. guianensis Baill. Hist. Pl. vii. 320 (Guiana) = Patima guinumensis Aubl.

S. macrophylla Steud. Nom. col. ii. $4 \$ 9=$ Pulicoureae sp.

S. Moralesii Grieb. Cat. Pl. Cub. 124 (Cubai) $=$ Lasianthus Moralesii Wright. 
S. Perrottetii A. Rich. Mém. Soc. Hist. Nat. Par. r. $228=$ Uncariae sp.

S. pumila Bartl. ex DC. Prod. iv. 440 .

Habitat, "in Peruviae montibus Huanoccensibus." The description reads, "caule herbaceo glabro, foliis oblongo-lanceolatis glabris sericeo-ciliatis, stipulis subulatis, capitulis pedunculatis axillaribus paucifloris" (Hb. Haenke).

The glabrousness and the subulate stipules suggest that the plant is not rightly placed in Sabicea. The material in the De Candolle herbarium, which I have examined, is very scanty, and inadequate for certain determination; but the leaf, which is quite glabrous except for the hirtous mid-rib, and has the veins flattened on the lower surface, is manifestly unlike that of any Sabicea species. It suggests Hoffmannia.

S. purpurea A. Rich. loc. cit. 228, and S. tomentosa A. Rich. loc. cit., both Brasilian plants, are unmentioned by Schumann in the Flora Brasiliensis. I have examined the respective types in the Paris Herbarium. Both have pedunculate capitate inflorescences - a very rare feature among the American species of Sabicea; and the linear to linear-subulate stipules do not suggest Sabicea. Both, I conclude, are species of Coccocypselum - the latter being clearly $C$. canescens Willd.

S. setosa A. Rich. loc. cit. is Flagenium setosum Wernham, comb. nor. Frutex ramulis pubescentibus; foliis oblongis $13 \mathrm{~cm}$. $\times 4 \mathrm{~cm}$. breviter acuminatis acutis basi rotundatis, petiolo 3-4 mm., supra nisi in vena centrali strigillosis glabris, subtus in venis deuse aliter sparsim brunneo-pubescentibus, stipulis subulato-acuminatis; floribus in fasciculis multifloris diametro ad $2 \cdot 5-3 \mathrm{~cm}$. confertis; calycis laciniis setaceis $7-8 \mathrm{~mm}$. sparsiuscule hirtellis ; corollae infundibularis glabrae tubo $1 \cdot 2 \mathrm{~cm}$. longo, ore ca. $8 \mathrm{~mm}$. lato, lobis oblongis $7-8 \mathrm{~mm}$. $\times 3 \mathrm{~mm}$.

Madagascar: Chapelier! Hb. Mus. Paris.

The contorted aestivation of the corolla, apart from any other character, excludes this plant from the genus Sabicea.

S. triflora DC. Prod. iv. 439. Triosteum triflorm Vahl, Symb. iii. 37 = Flagenium trifiorum Baill. (see Wernham, in Journ. Bot. li. (1913) 12). 



\section{SUMMARY OF COLLECTORS}

The numbers of the species are in round brackets ( ). Collectors' numbers are unbracketed; unnumbered specimens appear first in order of specific number; the rest follow in order of collector's number.

\section{CONTINENTAL AFRICA.}

AFzelius. (13), (39).

AllaRd. 146 (13), 343 (69).

BARTER. (39), (83), 1248 (84), 3282 (83).

Bates. 70 (83), 78 (87), 78a (87), $224(33), 398(75), 423(3 x), 536$ (62), $770(728)$.

Baumañ. 163 (83), 256 (70).

Beauvois, Palisot de. (83).

BrauN. 2 (83), 1936 (17).

Brown. 296 (15), 404 (83).

Bruneel. (83).

BuchHolz. (83), (87).

Bunting. (19), 28 (19).

Burton. (3T ?).

BÜSGEN. 239 (89), 451 (3), 484 (87).

Butaye. (60), 1459 (87), 1487 (60).

BÜTTNER. T3 (70), 263 (41), 437 (102), 440 (13), 443 (102), 447 (37), 749 (70).

Cabra. 93 (36).

Chevalier. $5069(13 \beta), 12390(104)$, 12687 (39), 14622 (87ß), 15343 (19), 17232 (37?), 17664 (19), 17722 (83).

Cuamssens. 113 (16), 161 (16), 173 (71), 648 (98), 711 (100), 742 (98).

CoNraU. 218 (41), 247 (40).

Cumains. 148 (83).

Demeuse. 261 (13).

DEW亡̀VRE. 145 (37), 287 (37), 1143 $(100)$.

Dinklage. 548 (78), 561 (87), 591 $(102), 707 \quad(83), 743 \quad(103 \beta), 788$ (87), 1011 (3), $1062(87), 1063$ (82), 1124 (99), 1153 (82), 1211 (82), 1284 (99), 1362 (87), 1363 (82), $1391(103 \beta), 1902(19 \beta), 1903(19 \beta)$, 2188 (19), 2224 (13), 2476 (19), 2509 (39), 2510 (76), 2563 (76).
DODD. 420 (83).

VON DOERING. 237 (83及).

DoN. (13), (39), (76).

Duparquet. (95).

Dupuis. (16).

DrBowsir. 157 (16).

ELLIOT, SCOTT. 3871 (39), 4175 (39), 5276 (13).

ELLIOTT, W. R. 77 (83).

ENGLER. 675 (17), 676 (17), 709 (17).

FARMAR. 234 (39), 379 (83).

FLAMIGNI. $73(98)$.

Foster. 185 (83).

GentiL. (103).

GILLET. 148 (69), 159 (13), Зड̃T (13), 775 (60), 1390 (13), 1749 (103), 1911 (71), 2024 (71), 2179 (18), 2572 (37), 2760 (13), 2779 (13), $3520(60 \beta)$.

Gontze. 209 (17).

Gossweiler. 601 (38).

Guizat. (78), (83).

HeNS. 139 (99).

HEUDELOT. 821 (13).

Hoj_land. 31 (83), 95 (90), 118 (84), 137 (83), $262(86)$.

Jespersen。 (98 $\beta$ ).

JoHnston. (83), (90).

JOLLY. 29 (83), 79 (78), 97 (13), 126 (97).

Kersting. 146 (70).

Kitsox. (83). 
KílaINe. (97), 100 (102), 123 (102), 377 (102), 766 (87), 771 (96), 812 (87), 1532 (87), 2531 (96), 2684 (83), 2808 (83), 2847 (83), 2869 (87), 2918 (83).

KRAUSE. (8:3).

KRICKELS. (18).

Laurent, F. \& MI. (13), (70), (71) (83), (983), (105ß).

LAURENT, MI. 902 (98), 1597 (100). LECOMTE. (83), C 92 (13), C 99 (13), E 110 (83).

IEDERMANA. 595 (3), 714 (87), 864 (78), $918(103), 1014$ (83), 1515 (85), 2450 (93), 2655 (72), 6066 (83).

LescrauWAEt. 364 (83).

MACGREGOI, 35 (83 $\beta)$.

Maclád. 358 (39).

Malchair. 328 (98 $\beta)$.

Many. (6), 41 (87), 53 (83), 862 (76), 918 (7), 1723 (88), 1766 (102), 1866 (78), $2195(87)$.

MildBRAED。 2787 (83), $2823(100 \gamma)$, 2983 (83), 3661 (60), 3701 (18), 4711 (20), 5122 (103), 5141 (103), 5416 (98), 5422 (72), 5424 (89), 5433 (94), 5573 (83), 5619 (82), 5621 (98), 5905 (2), 6201 (78), 6288 (7), 6851 (87), 7011 (4).

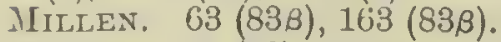

Miller. 50 (92), 68 (92), 70 (92).

Millsox. (83B).

Moller. 2 (6).

Moloney. 25 (83).

MUPfiY. 679 (19).

Nachtigall. (83).

POGGE. 979 (98), 981 (16), 1041 $(72 \beta), 1162(72 \beta), 1196(72 \beta)$.

Preuss. 15 (78), 70 (83), 150 (41), 159 (83), 278 (78), $325(41), 872 \mathrm{~A}$ (13), 1151 (83), 1334 (83).

P'UNCH. 63 (83B).

PuRnIE. (76).

PYNAERT. 12 (98?), 42 (13), 241 (83), 510 (98), $515(13 \beta, 21), 579$ (98), 1228 (98), 1268 (37), 1710 (98).
Quintas. 18 (74).

READE. (39).

REDER. 1154 (13).

Roвb. (83), (96).

ROWLAND. (\$33), 55 (83).

Rudatis. 21 (83), 44 (77), 52 (103) 57 (41), 71 (86).

SAPIN. (61), (71), (101), Y34 (13).

Schaefer. $76(73)$.

SCHLECHTER. 12658 (133), 13024 $(83 \beta)$.

Smeathinann. (13).

Smith, Ciristian. 59 (22).

Smithe. 55 (13).

Soyaux. 16 (82), 220 (78).

StaUdF. 1 (103), 159 (103), 237 (14).

Stuhliann. 8775 (63), 8872 (17).

TALBOT. (83), (90), $114 a(83), 228$ (103), 249 (5), 255 (77), 259 (3), 1040 (41), 1367 (91), 2032 (88), 2033 (91).

Tessmann. 654 (98), 716 (103), 751 (94), $883(83)$.

TIIOLLON. 14 (96), 60 (96), 104 (16), 117 (102).

THOM I'SON. 46 (37 ?).

Thonser. 202 (17).

VANDERYST. (13), (60), (71)

VOGEL. 35 (8:3), 87 (39), 88 (87).

VOLKEXS. 133 (17).

WeLWitsch. 3165 (69), 4744 (38), $4745(38)$.

Whyte. (19), (76ß).

WiNKLER, 27 (41), 39 (83), 1027 (86).

ZnNKer. 204 (72ß), 675 (13), 719 (13), 1041 (2), 1140 (83), 1141 (87), 1816 (41), 1818 (82), 1820 (103), 1821 (97), 2053 (78), 2095 (42), 2174 (3), 2968 (72), 2997 (83), 3232 (103), 3877 (83), 4020 (14), 4070 (62), 4072 (14), 4414 (42), 4567 (14), 4568 (83).

ZeNkEI \& S'TAUdT. 303 (83).

Zimmernans. 40 (17), 107 (17).

\section{MADAGASCAR AND MASCARENE ISLANDS.}

Paron. (45), 298 (45), 5736 (43), $6238(43)$.

BERNARD. (45).

BERNIER. $271(45)$.

HoIviN. $1767(45), 2064(43,44)$.
BHEON, 27 (45).

Catat. $1722(45)$.

Cifapelier. (45).

Elciot, Scots, 1772 (15). 
Flacourt. 98 (46), 126 (46). Forbes. (45).

Gerrard. 24 (45).

Hildebrandt. 3024 (43).

Hilsenberg. (45).

Humblot. 69 (45), 213 (105).

Lastelle. (45).
LYALL. (45).

Mocquerys. 162 (45), 264 (45).

Petit-Thouars. (43), (45).

PoIvre. (45).

RichaRd. $3 \pm$ (45), 211 (43), 636 (43).

Thompson. (45).

\section{AMERICA.}

APPUN. 328 (53), 359 (83).

AUBLET. (47), (66).

BAKER. 73 (66).

BALBIS. (65).

BaLl. (10).

BANG. 384 (26), 1380 (24).

Bertero. (65), 2703 (33).

Billberg. (65 $\beta)$.

Branchet. (51), (57), 53 (51), 85 (51), 1175 (51), 3004 (65y), 3124 (51).

BOOG. (51).

Bowie \& Cunningham. (51).

Broadway. 2226 (53), 3337 (65), $3338(65 \beta)$.

BuRCHELl. 774 (51), 1804 (51), 2672 (51), 2960 (51), 3155 (65), 3175 (65), 5136 (57), 6532 (57), 7035 (57), 8061 (57), 8383 (57), 9271 (51), 9346 (66), 9429 (66), 9989 (66), 10,038 (66).

CASARETTO. 2245 (51).

Castelinau. (12), $(65 \gamma), 622(65 \gamma)$.

CAvaNilles. (32).

Claussen. (57), 271 (57), $560(57)$, $558(57), 609$ (57), $673(57)$.

Custopro. 98 (51).

DofLLINGER. (51),

DOMBEY. 561 (32).

DUPRÉ. (51).

DUSÉx. 4469 (65).

EGGERS. 1065 (65).

FENDLER. 180 (65B), 181 (9), 425 (53).

FLEISCHER. (51).

FORREST. 38 (51).

FREYREISS. (51).

FriedRICHSTAHL. (65B).

FROHICH. (51).

FunCKE \& SCHLIM. 624 (34).
GALEOTTI. 2662 (35).

GARDNER. 1338 (51), 1697 (51), 2886 (57), 3225 (57), 5486 (51).

GaUdichaUd. 630 (51).

Gaumer. 1432 (68).

GAY. (51).

GrazioU. (57), (66), 714 (51), 8740 $(51,54), 14912$ (57), 19420a (29), $19435 a(49,65 \gamma)$.

GLOCIERR. 561 (51), 591 (51).

Goudot. (65).

GUILLEMiN. 129 (51).

GUILLOT. (51), 3224 (51).

GundLaCH. 1474 (65).

Givynne-Vaughan. 25 (49).

HANCOCK. 282 (53).

Harris (Fl. Jam.). 9246 (33), 10278 (33), 10561 (33).

HART. 144 (31).

HAYES. (65ß).

Hilaire, St. 59 (51), 182 (51), 283 (57), $980(65 \gamma), 2281$ (57).

Hostitann. $50(53)$.

Humboldt (\& Bonpland). (65).

JELSKI. (47), (65).

JENMAN. 1724 (53), 1775 (53), 3698 (64), 4711 (64).

KALBREYER. 1837 (1).

KARSTEN. (34).

КосH. $92(49)$.

KRUG \& URBAN, Hb. 1074 (64).

KUNTH, Hb. (47), (65).

KUNTZE. 1995 (65ß).

LEBLOND. (47).

LECHLER. 2510 (65).

LEDRU. (47).

LEGUILLON. (47).

LEHMANN. 4001 (58).

IJRPIEUR. (65), 101 (47), 102 (47).

LHOTSKY. 558 (51).

Liebiann. 43 (35). 
LINDBERG. 718 (65).

LINDEN. 1498 (31).

LoCKHART. 350 (65).

Macfadyen. (33).

MIAcrae. (51).

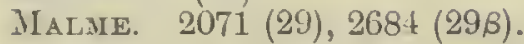

MaRTIN. $63(66, \beta), 82(47), 95$ (47), $246(65)$

Martius. (51).

MIASSOX. (33).

Matтhis. (58), 1951 (32).

Mellinon. 186 (53).

Miers. 3154 (51).

Miquel. (53).

Moone, SPENCER. 472 (29), 794 $(29 \beta), 785(80)$.

MORICAND, Hb. (47), (66), 179 (65).

Mosen. $3416(65)$.

MŨ̈LLER. 128 (65).

NADEAUD. (51).

PARKER. (64).

Patris. (47), (66).

Pfarce. (25), (27)

Perrottet. (47), (66).

Piтtikil. 1208 (65), 2904 (11 ?), 4025 (11), 6712 (11), 10297 (65).

Pomprig. (65B), 43 (32), 379 (65), $1226(32), 1821(65), 2514$ (49).

PoIrL. 148 (57), 864 (51), 957 (57).

Poithau. (47), (65), (66).

Purdie. (33).

RADDI. (51).

REGNELL. 92 (51), 1016 (57).

RichaRd. (32), (47), (57), (65), (66).

RidLey, Lea, \& RaMIAGE. (51).

RIEDEL, 116 (57), 623 (51), 2067 (57).

RIEDLÉ. (65).

Rivero. 379 (65).

Robert. 687 (30).

ROTHFRY. 231 (47).

Ruiz \& Pavon. (32), (58), (65).

RUSBY. 1905 (57).

SAGOT. 309 (47), 880 (65).
SALDANHA. 439 (51).

SALZMANA. (51), T (51)

SCHENCK. 1697 (51), 3379 (57) 3776 (51), $4279(50)$.

SCHLIM. 697 (31).

Schomburgk. (59), 25 (53)， 533 (64), 901 (53), 1333 (53).

SCHWACKE。 $3452(64), 4755(51)$

SELMANN. $128(65), 1073(65 \beta)$.

Sello. $223(65 \gamma), 299(65 \gamma), 323$ (51), $381(65 \gamma), 732(65 \gamma), 768$ (51), $804(51), 959(51), 1072(65 \gamma)$, $1076(51)$.

SIEBER. 327 (65).

Sintenis. $263(65), 1819$ (65).

Sirti, H. H. $1834(31,65)$.

SODIRO. (65).

Sprague. 43 (55).

SPRUCE. (49), (58), 320 (48), 684 (48), $1281(64), 3275$ (64), 4370 (25), 4837 (65).

StallL. $376(65)$.

SWARTZ. (33)

TATE. $212(65)$.

TONDUZ. $6712(658), 9186 \quad(65)$, 9955 (658), 13363 (65).

Trail. 389 (49), 390 (79), 391 (67).

Triana. (34), 681 (31), 717 (81), 1752 (31), 1755 (34), 1756 (56).

Trinitat., Hb. 1793 (65).

Türekherm. 7749. [J. D. SMitri]. (65).

ÚLE. 182 (65), 3803 (51), 5117 (49), $5118(65 \beta), 5669$ (12).

VAuthier. 41 (51), 98 (51).

WARMING. (54), (65), 105 (57).

WEBERBAUER. 4568 (58).

WEDDEL. 295 (51), $2563(57)$

WIDGREN. (65), 119 (51), 535 (65), 1047 (51).

Willians. 173 (57), 446 (23), 590 (23).

WILSON. (33), 232 (65).

WRIGIIT. 655 (33).

WULLSCIILAEGEL。 253 (53). 


\section{N D E X}

(Synonyms and vnlgar names are in italics)

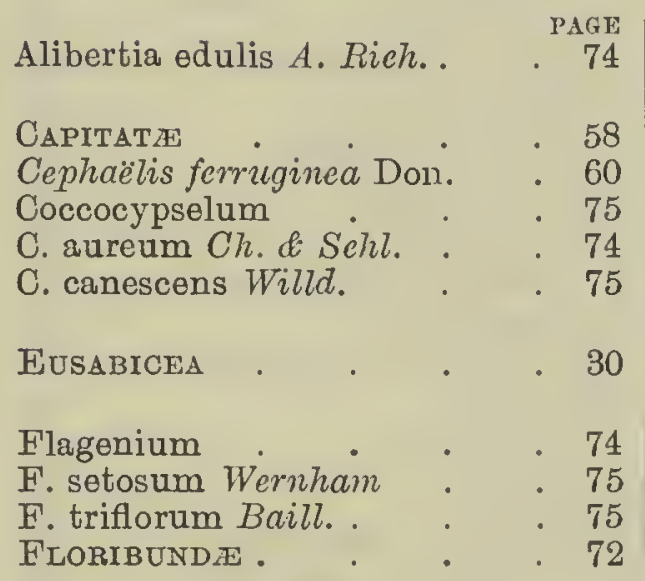

Lasianthus Moralosii Wright . 74 LAX环 . . . 30

Manettia asperula Ball . 30 Modila Dila . . . . . 32 Mokessé . . . . . 71 Ondvikoni . $\quad . \quad . \quad .69$

Paiva verticillata Vell. . . 55 Patima guianensis Aubl. $\quad$. 74 Phaoloi. . . . . 63 Po-ri . . . . . 55

SABICEA. . . . 20

S. acuminata Baker . . 44

S. affinis Dc Wild. . . . 32

S. amazonensis Wernham • 47

S. angolensis Wernham . . 42

S. angustifolia Boivin . • 45

S. arborea K. Schum. . . 54

S. aspera Aubl. . . .49, 54, 56

S. asperula Wernham . . 30

S. aurea Steud. . . . 74

S. Barteri Wernham . . 64

S. Batesii Wernham . . 53

S. bicarpellata K. Schum. - 27

S. boliviensis Wernham . . 37

S. brachiata Wernham . . 68

S. bracteolata Wernham . . 73
S. brasiliensis Wernham PAGE

S. brevipes Wernham . . 58

S. brunnea Wernham . . 65

S. Burchelii Wernham . . 49

S. calycina Benth. . . . 63

S. cameroonensis Wernham . 35

S. camporum Spragne . 50

S. cana Hook. . . 47, 51, 52

S. capitellata Benth. . . 65

S. cauliflora Hiern . . . 28

S. cinerea Aubl. . . 46,47

S. cinerea Karst. . • . 41

S. colombiana Wernham. . 39

S. composita Wernham . . 64

S. costaricensis Wernham - 31

S. erinita A. Rich. . . . 74

S. cruciata Wernham . . 68

S. cuneata Rusby . . . 37

S. Dewevrei De Wild. . . 71

S. Dewildemaniana Wernham . 42

S. Dinklagei K. Schum. . . 70

S. discolor Stapf . . . 35

S. diversifolia Pers. • $\quad 45,47$

S. dubia Wernham . $\quad .53$

S. Duparquetiana Baill. . • 69

S. edulis Seem. • • . 74

S. entcbbensis Wernham : 33

S. erecta Rusby . . . 36

S. eriantha DC. . . . 48

S. ferruginea Benth. $\quad .60$

S. flagenioides Wernham. . 57

S. flavida Krauso . . . 52

S. floribunda K. Sehum. . . 72

S. fulva Wernham . . . 66

S. geantha Hiern * * . 29

S. geophiloides Wernham : 60

S. gigantea Wernham • . 71

S. gigantostipula K. Sehum. . 27

S. Gilletii De Wild. . . 58

S. glabrescens Benth. . . 54

S. glomerata Wernham . 50

S. gracilis Wernham : 60

S. grisca Ch. \& Schl. . $\quad 47,48$

S. guiamonsis Wernham . . 52

S. guianensis Baill. . . 74 


\begin{tabular}{|c|c|c|c|}
\hline & & & \\
\hline UT - • & & S. setosa A. Rich. & \\
\hline Ternham & 29 & S. seua Wernham & \\
\hline birsuta $H . B . \& K$. & 55 & S Smithii Wernhan & \\
\hline hirta $S w$ & & S. speciosa $K$. Sehum. & \\
\hline milis S. Moore & & S. speciosissima $K$. Sehum. & \\
\hline ata $K$. Schum. & & S. stipularioides Wemham & \\
\hline K. Sehum. & & S. subinvolucrata Wernham & \\
\hline olbeana Büttn. & & S. Talbotii Wemnam & \\
\hline rntham & & hapensis Krause & \\
\hline S. $L$ r -10 & 60 & mentosa A. Rich. & \\
\hline trild. & 70 & railii Wernham & \\
\hline ham & 33 & S. Trianae Wernhan & \\
\hline Wernham & 50 & S. trichoehlamys K. Schum. & \\
\hline & & & \\
\hline 11. & 74 & igemina $K$. Schum. & \\
\hline emham & 62 & a Pers. & \\
\hline 7num. & 44 & Wernham & \\
\hline$m$ & & a Wernham & \\
\hline am. & 5 & $u t h$. & \\
\hline . & 33 & S. & \\
\hline & 47 & Verntuan & \\
\hline & 39 & S. & 43, \\
\hline & 7. & tricha Wernham & \\
\hline vo-granatensis $K$. Selu & & Sabi- & \\
\hline lam . & & elda. & \\
\hline lam. & 30 & inerea Sw. & \\
\hline & & & \\
\hline lam & 31 & mbellata Ruiz \& Pav. & \\
\hline & 57 & dia aspera Willd & \\
\hline zum. & 5 & M & \\
\hline & 3 & ia Spr. & \\
\hline nham & 6 & iantha Dietr. & \\
\hline Rieh. & 7 & irsuta Spr. & \\
\hline & & S. hirta Willd. & \\
\hline eudocapitellata Wernha & & S. villosa Willd. & \\
\hline & & SHSSILES & \\
\hline Rich. & 75 & Serua & \\
\hline & 6 & Stipularia . & \\
\hline Wernt & 6 & StIPULARIOPSIS & \\
\hline & & & \\
\hline Biittu. & & Triosteum triflorum Vahl & \\
\hline $\begin{array}{l}\text { S. segregata Herrn. } \\
\text { S. setiloba Wernham }\end{array}$ & & Voa Scira & \\
\hline
\end{tabular}

\section{B.AY.1914}

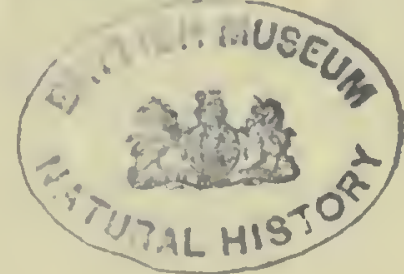




\section{PLATES}




\title{
EXPLANATION OF PLATE I
}

\author{
s. umbrosa Wermham
}

1. Portion of shoot, $\times !$

ง. orientalis Wernham

2. Portion of shoot, $\times \frac{1}{2}$.

3. Stipules, $a$, interior, $b$, exterior, $\times \stackrel{\text { ?. }}{ }$

4. Portion of inflorescence in young fruiting stage, natural size.

5. Wlower, natural size. 


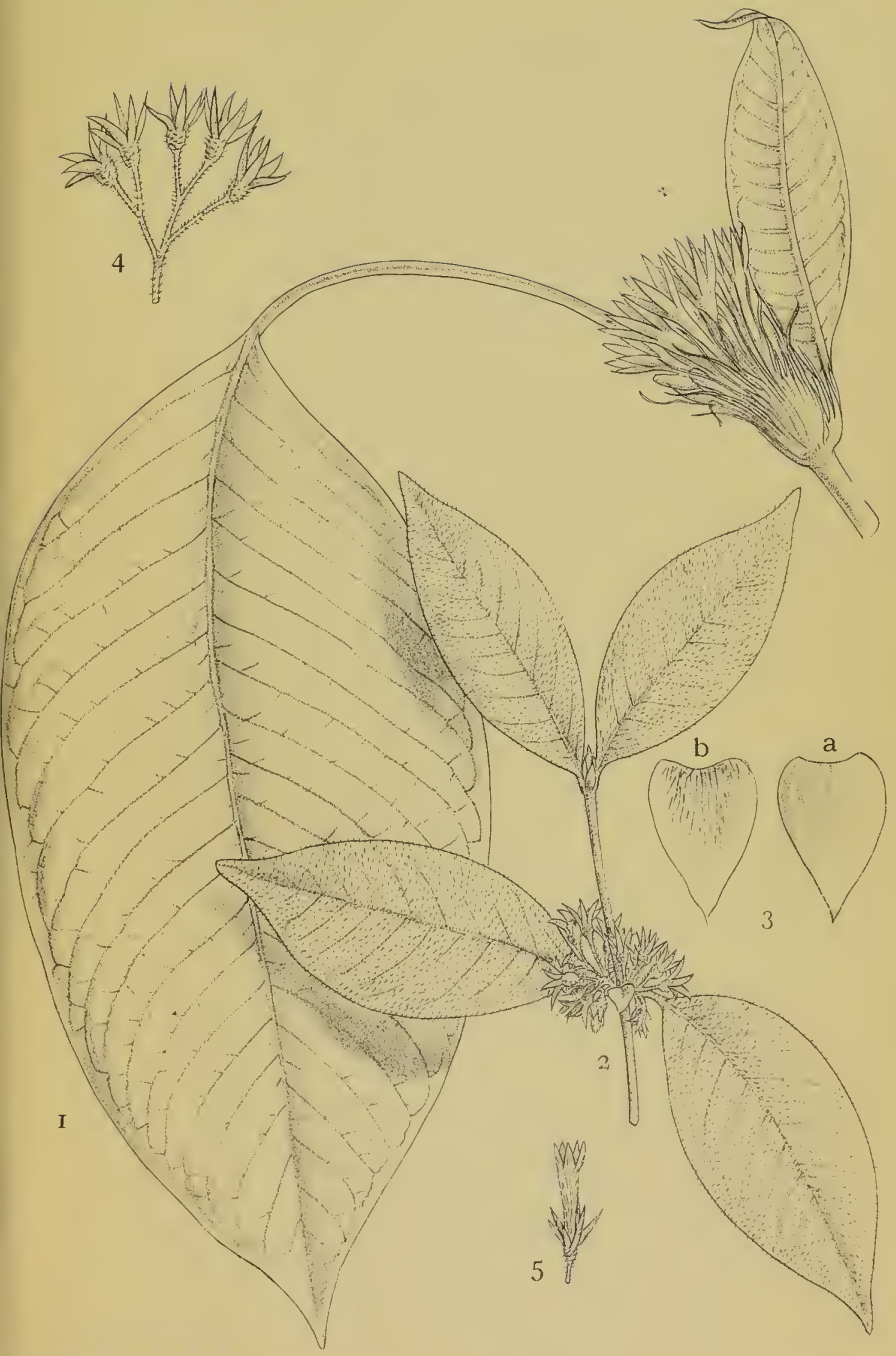

J. N. Fitch det. \& lith.

I, S. umbrosa. $2-5$, S. orientalis. 


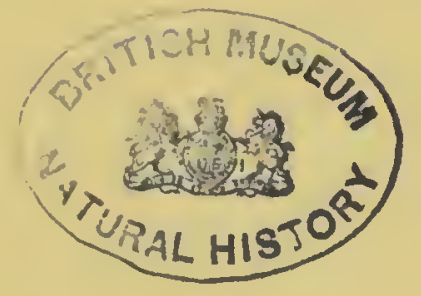



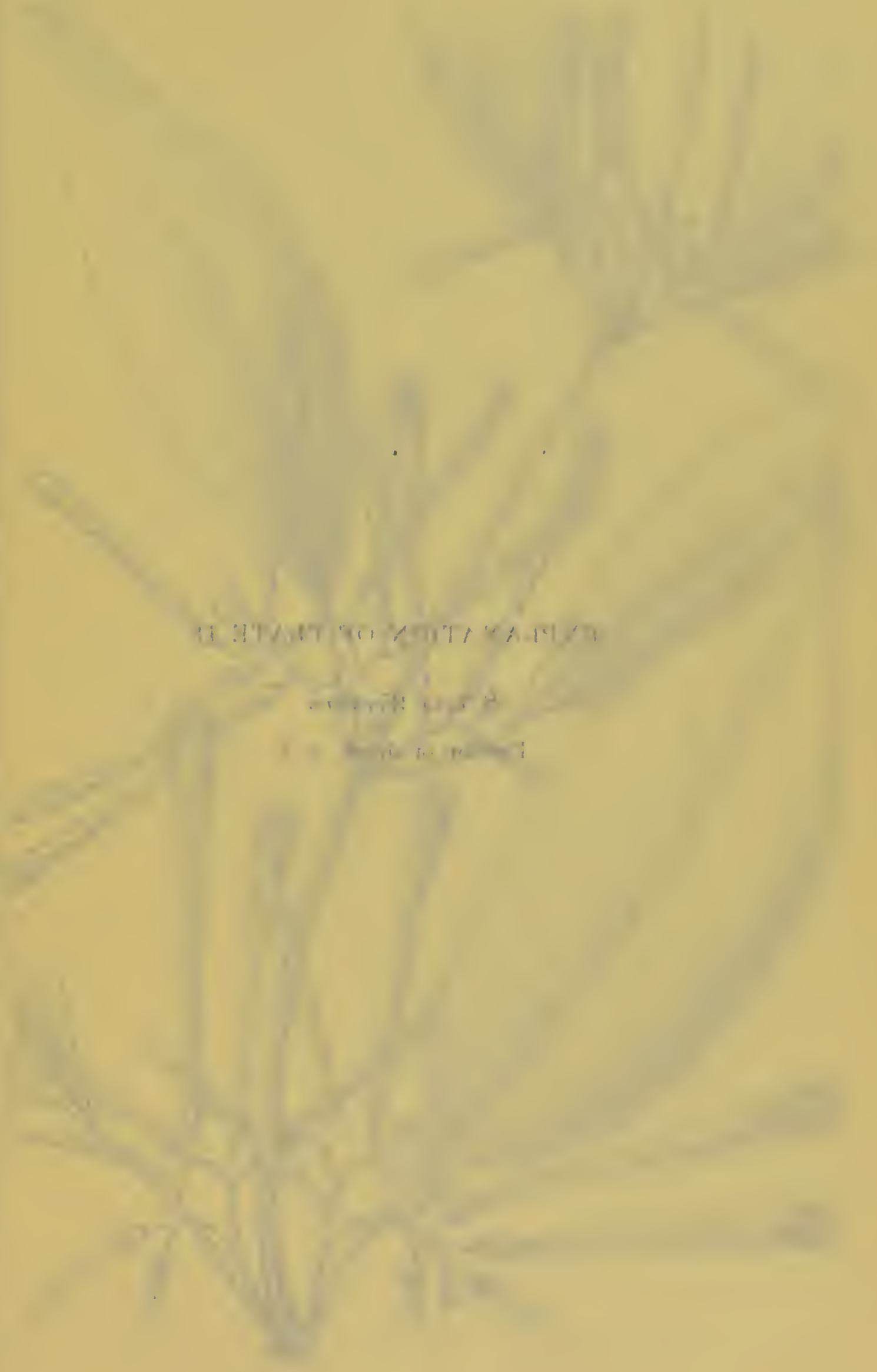
EXPLANATION OF PI,ATE II

S. laxa Wernlaam

Portion of shoot, $\times 2$. 


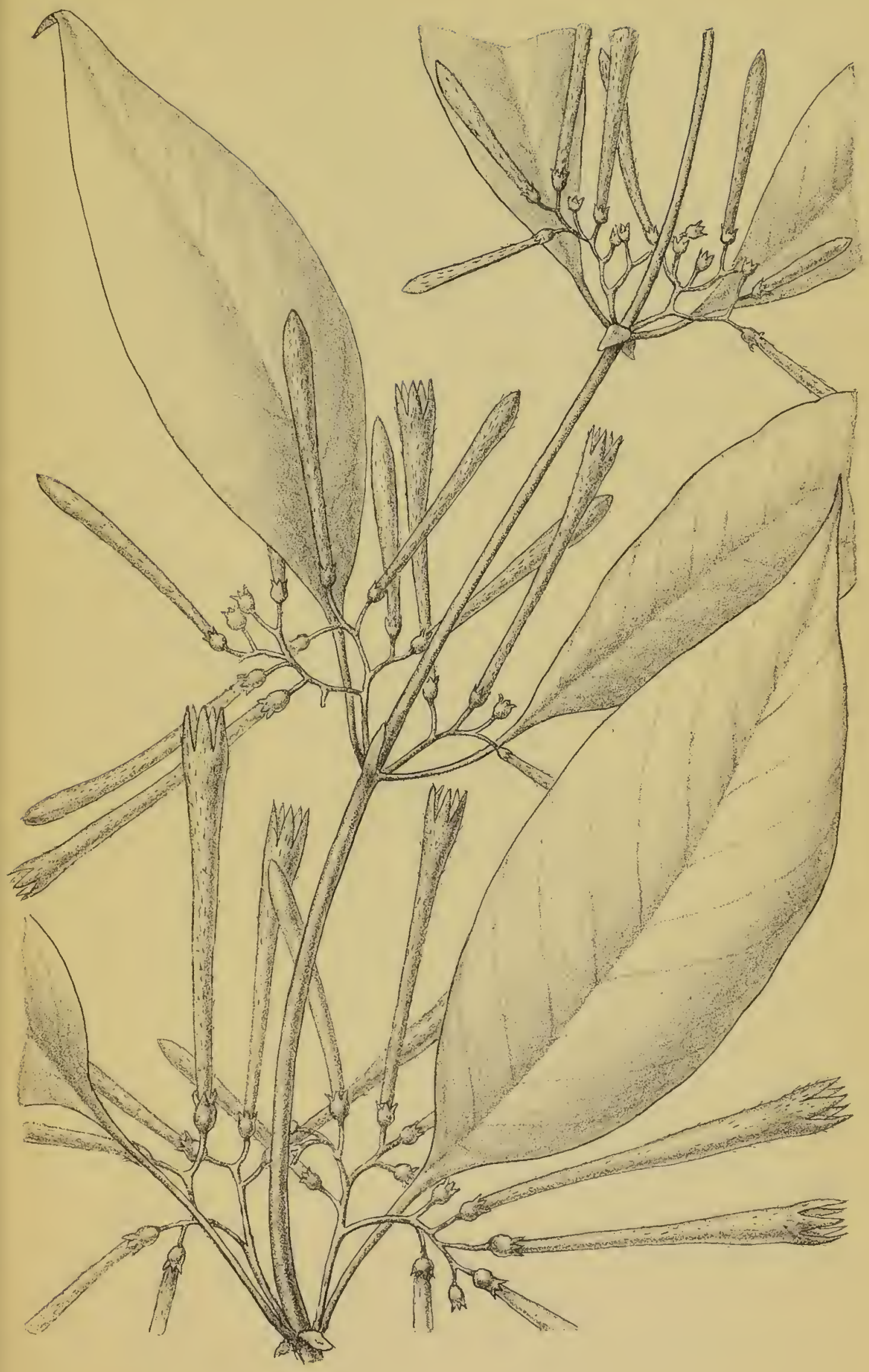

P. Highley del. \& lith.

S. Laxa. 


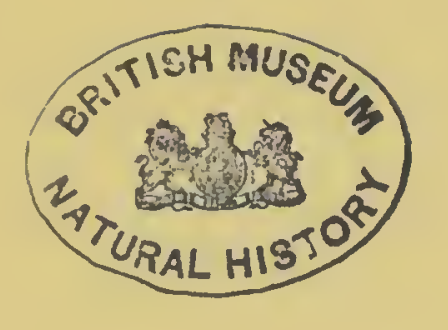



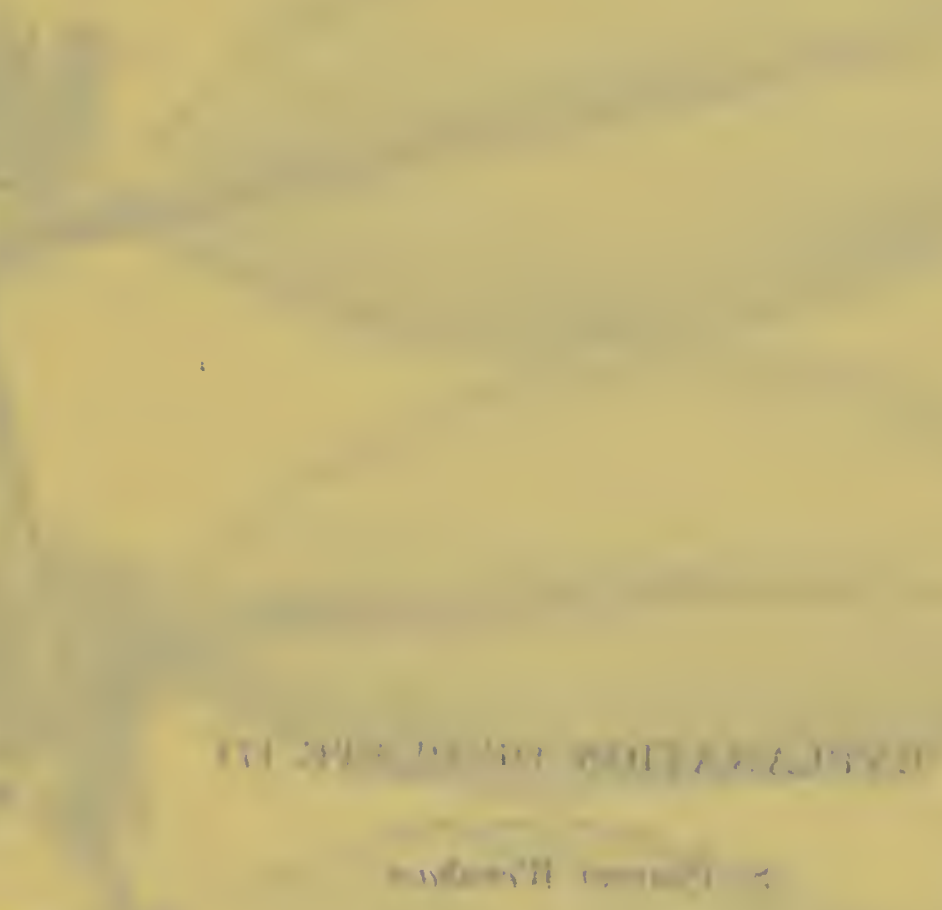
CXYLANAILON OF PLATE III

\section{S. Pearcei Wernham}

1. Portion of shoot, natural size.

S. subinvolucrata Wernham

2. Portion of shoot, natural size.

3. Hlower, $\times 3$. 


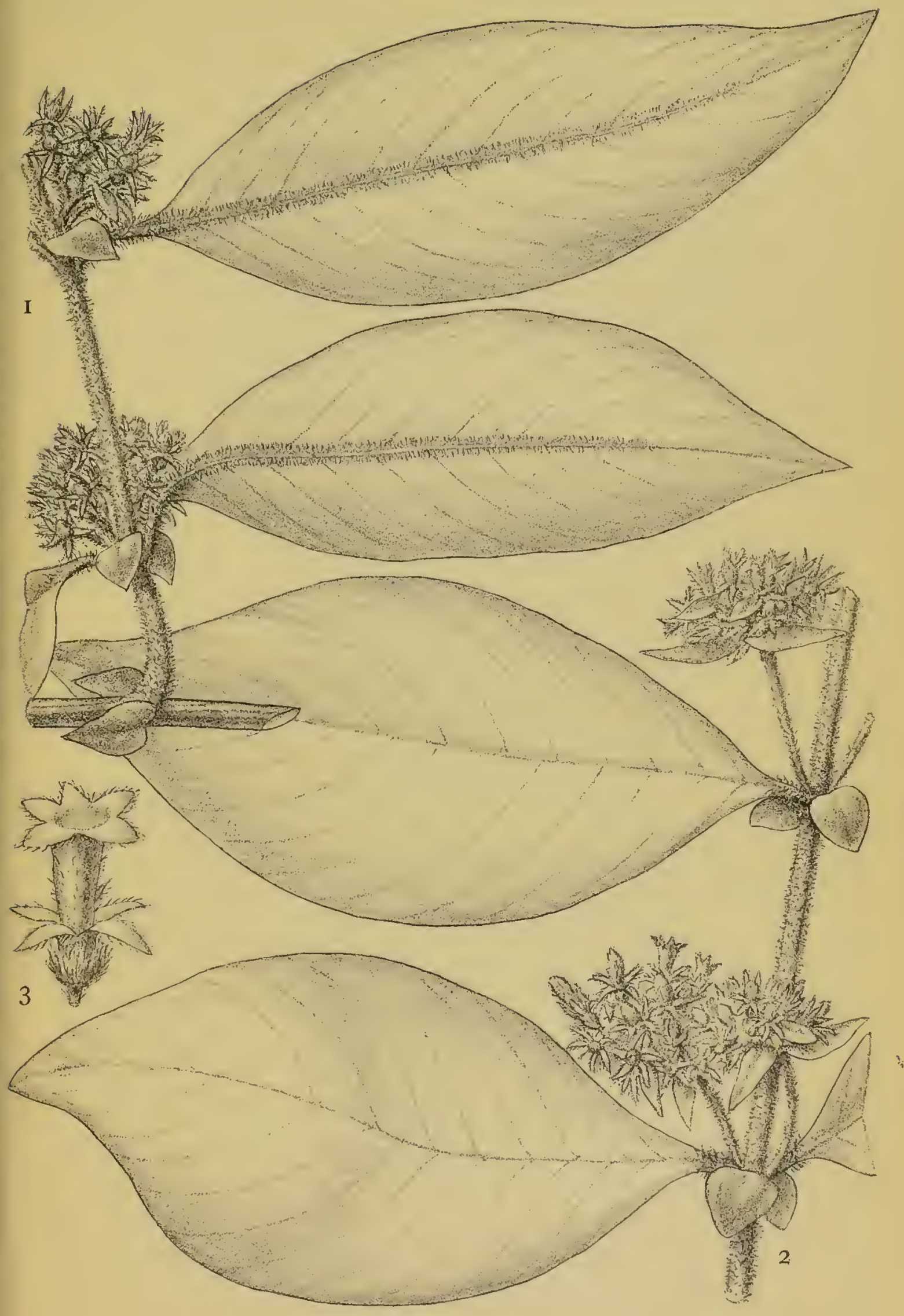

P. Highley del. \& lith.

I, S. Pearcei. 2, 3, S. subinvolucrata. 



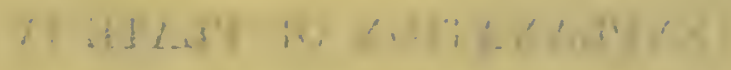

w...L $>$ it, +..., is =

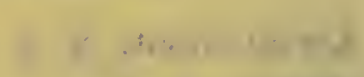

$-\ldots+2 .+1$.

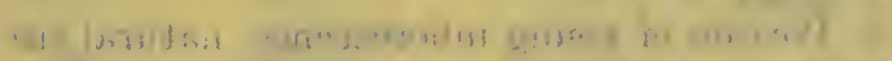

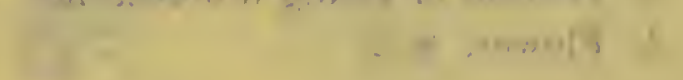

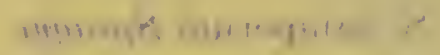

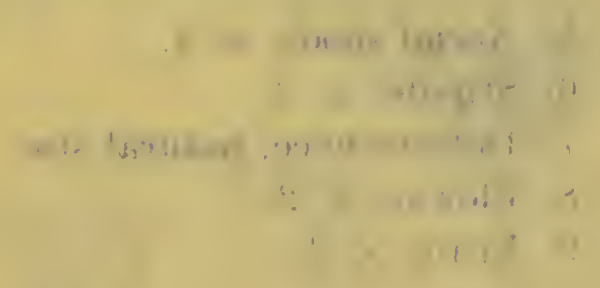




\title{
LAPLANATIUN OF PLATE IN
}

\author{
s. Noorei Weruham
}

1. Aerial shout, $\times \frac{1}{3}$.

2. Stipule, $\times 2$.

3. Portion of young inflorescence, natural size.

4. Hlower, $\times 2$.

S. camporum Sprayne

5. Aerial shoot, $\times \frac{1}{3}$.

6. Stipule, $\times \stackrel{2}{ }$.

7 . Inflorescence, natural size.

8. Flower, $\times 2$.

9. Fruit, $\times 2$. 


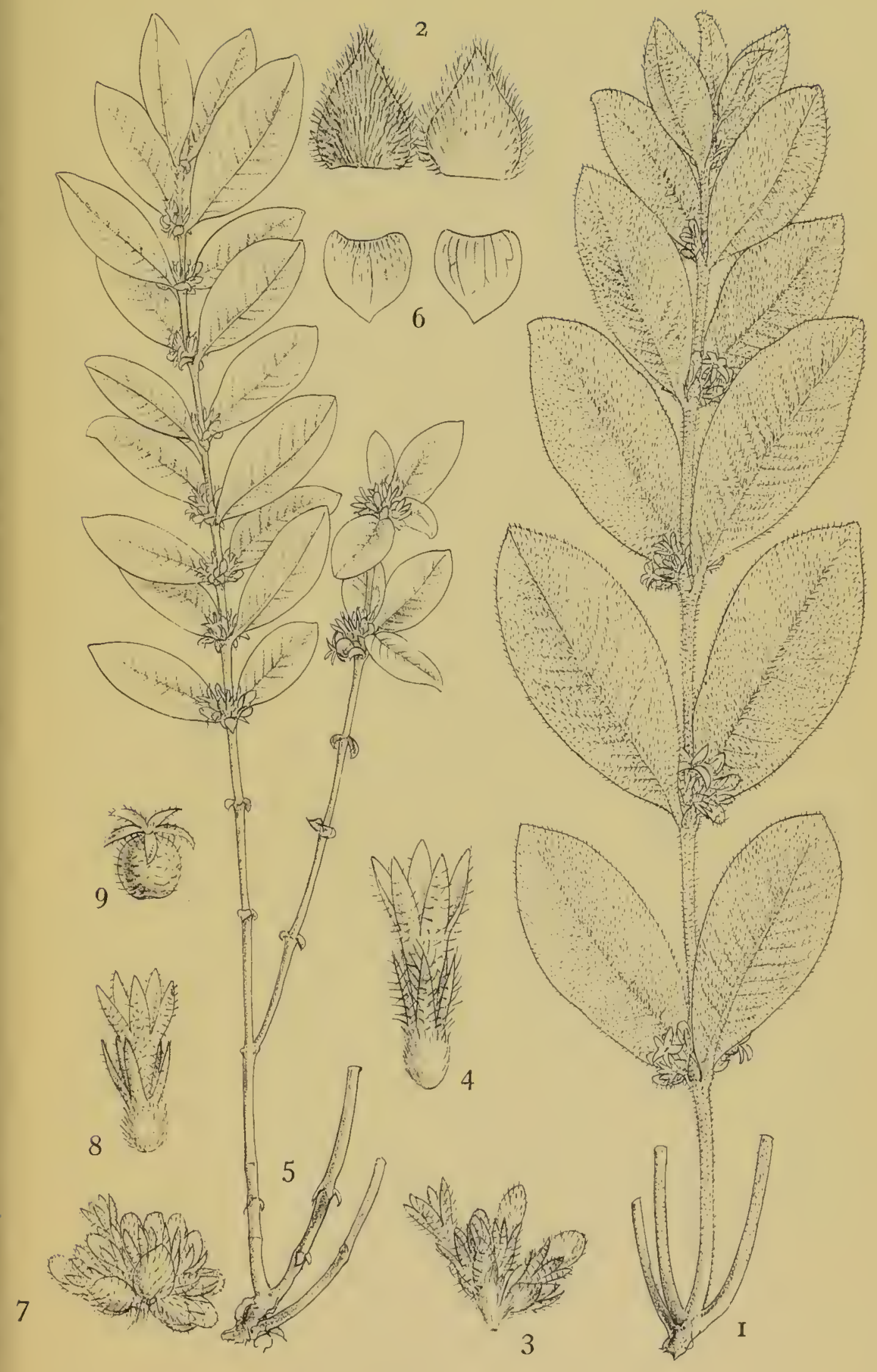

J. N. Fitch del. \& lith.

I-4, S. Moorei. 5 -9, S. camporum. 


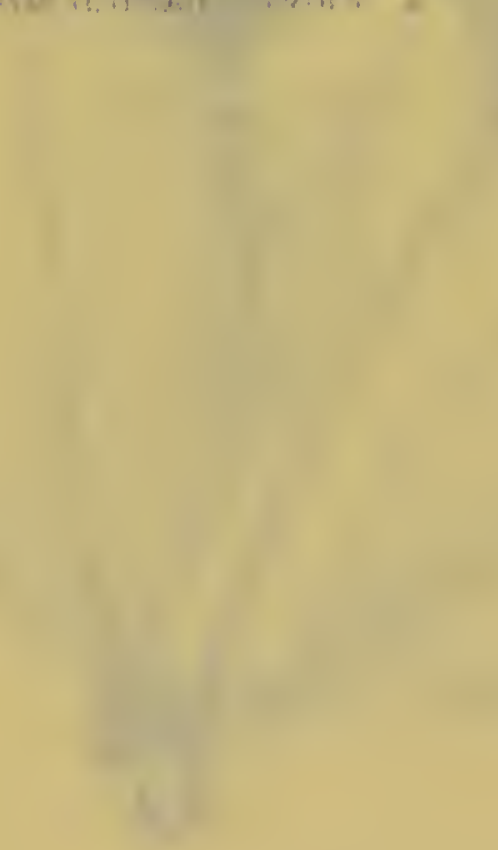
EXPLANATIUN OF PLATE V

S. mexicana Wernhum

1. Portion of shoot, $\times \frac{1}{2}$.

․ Hlower, natural size.

s. amazonensis Weruhum

3. Portion of shoot, $\times \frac{1}{2}$.

4. Nlower, natural size. 


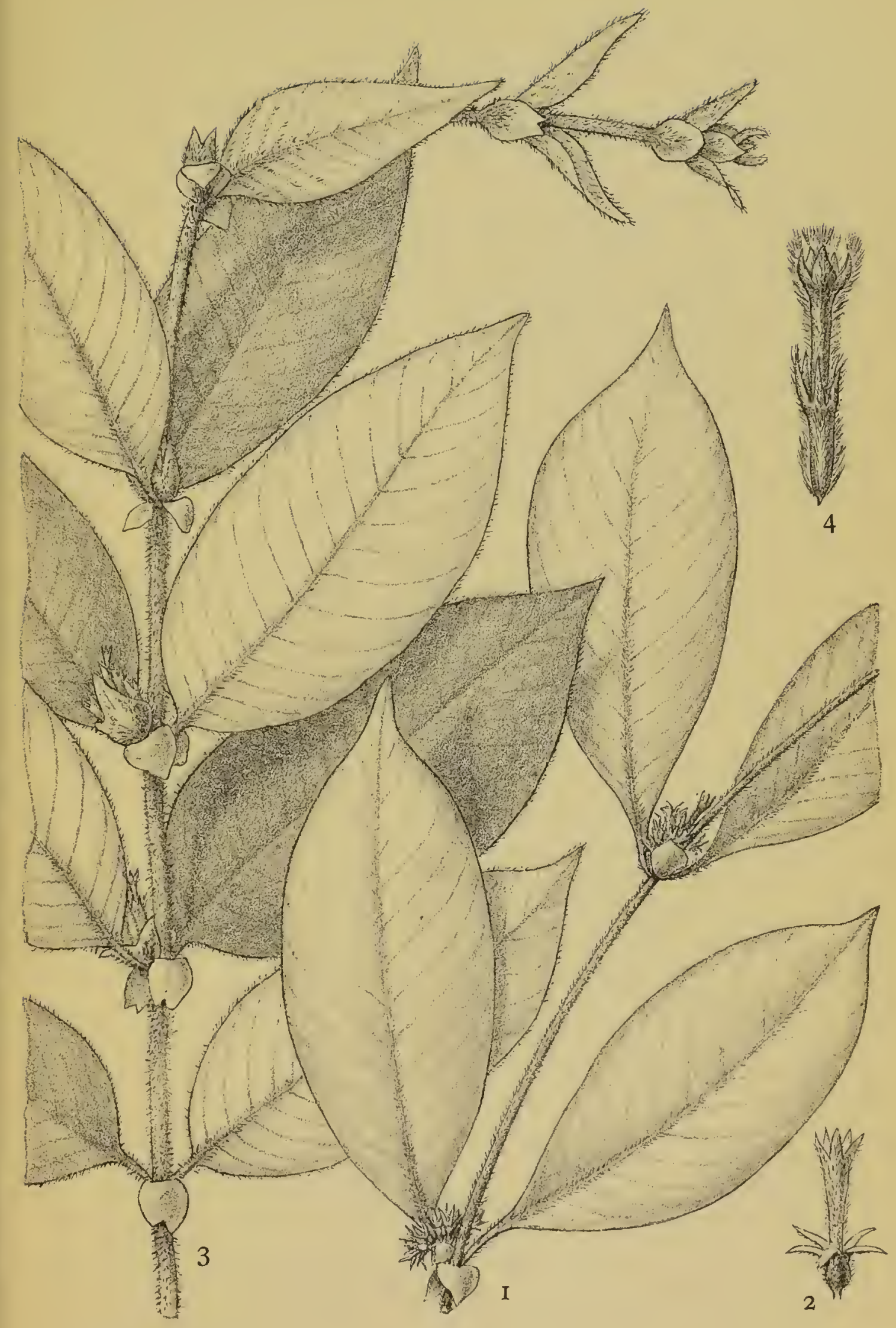

P. Highley del. \& lith.

I, 2, S. mexicana. 3, 4, S. amazonensis. 


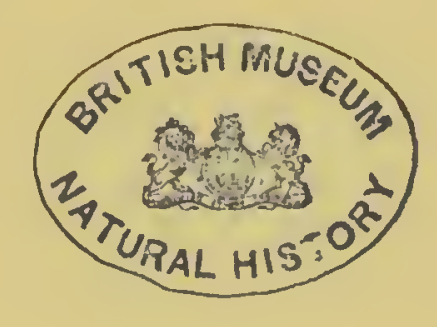




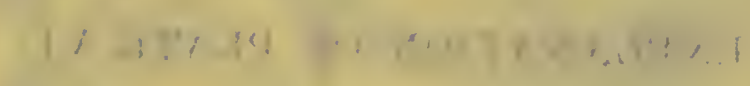

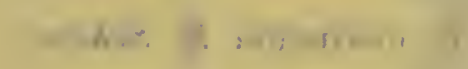

$13 \times-4:+1 ;$

7. . $\therefore \times, n=-i$.

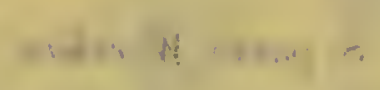

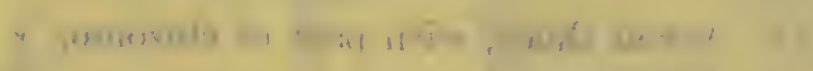

-

; $3 \times 1, \ldots+1$ 


\section{EXPLANATIUN OF PLA'I'E VT}

S. medusula K. Schum.

1. Acrial shoot, $\times \stackrel{1}{.}$.

2 . Stipule, $a$, exterior, $b$, interior, $\times 2$.

3. Flower; $\times 3$.

\section{S. parva Werwhem}

4. Aerial shoot, with part of rhizome, $\times$ 采.

5. Stipule, $\times 2$.

6. Flower, $\times 3$. 


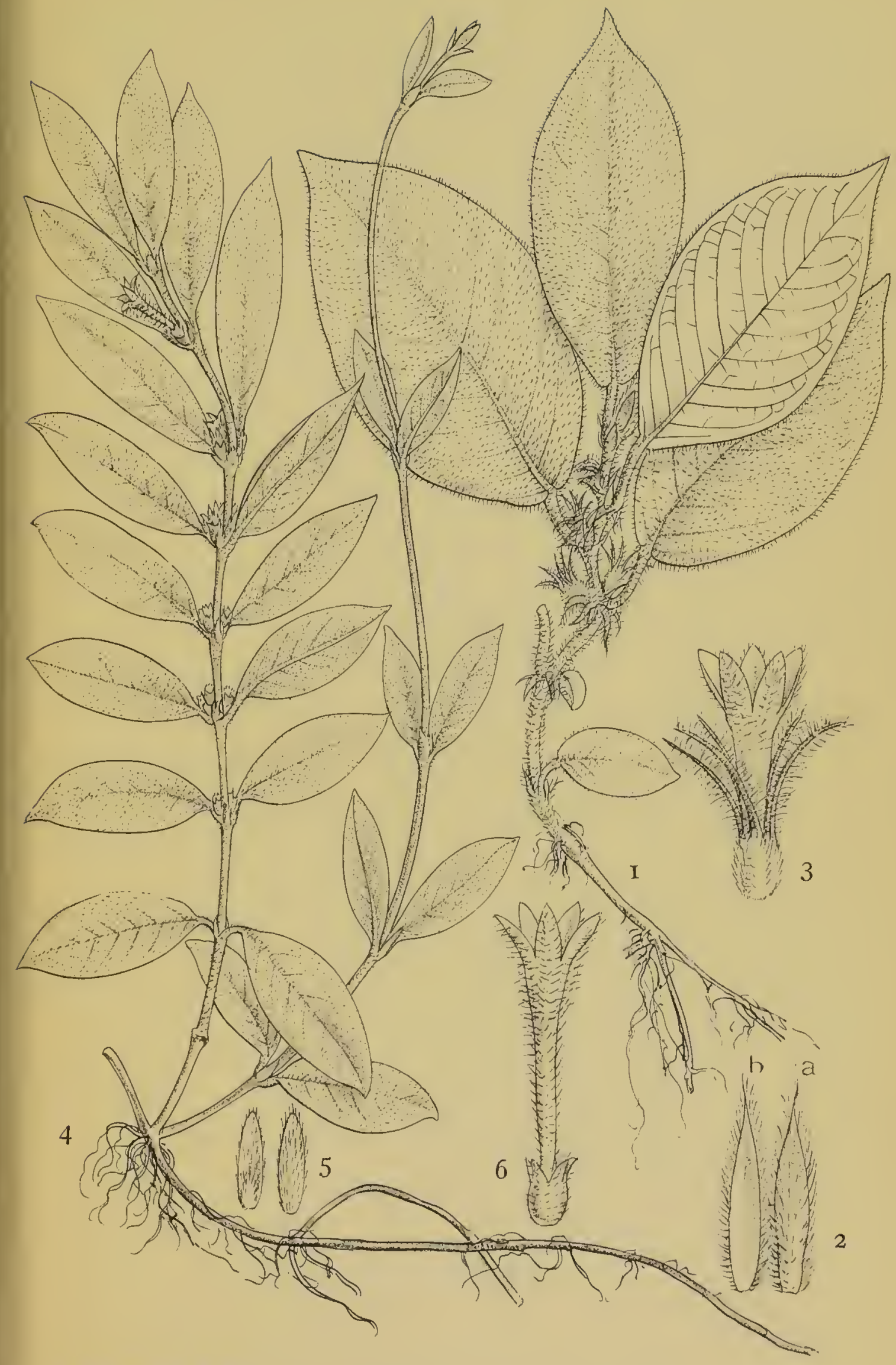

J. N. Fitch del. \& lith.

I -3 , S. medusula. 4-6, S. parva. 


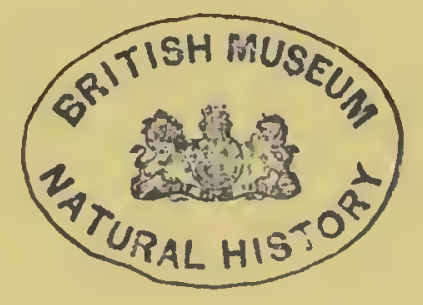




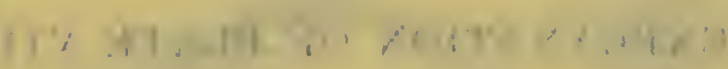

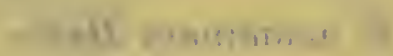

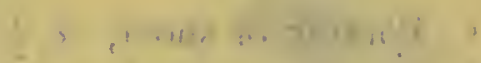

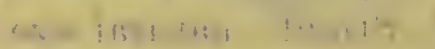

$$
\begin{aligned}
& \text { at fore: }
\end{aligned}
$$

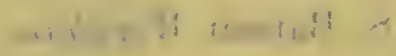

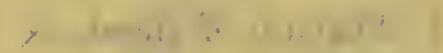

$$
\begin{aligned}
& \text { on. init, . If hel. }
\end{aligned}
$$

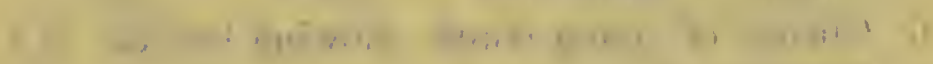

$$
\begin{aligned}
& \text { 1. ....? }
\end{aligned}
$$




\section{EXPLANATION OF PLATE VII}

S. acuminata Baker

1. Portion of shoot, $\times \stackrel{1}{\text {. }}$

2. Stipule, natural size.

3. Flower and bud, natural size.

S. Batesii Wernham.

4. Portion of shoot, $\times \stackrel{1}{2}$.

5. Stipule, natural size.

6. Cluster of young fruits, showing hracts, $\times 2$.

7. Hruil, $\times 2$. 


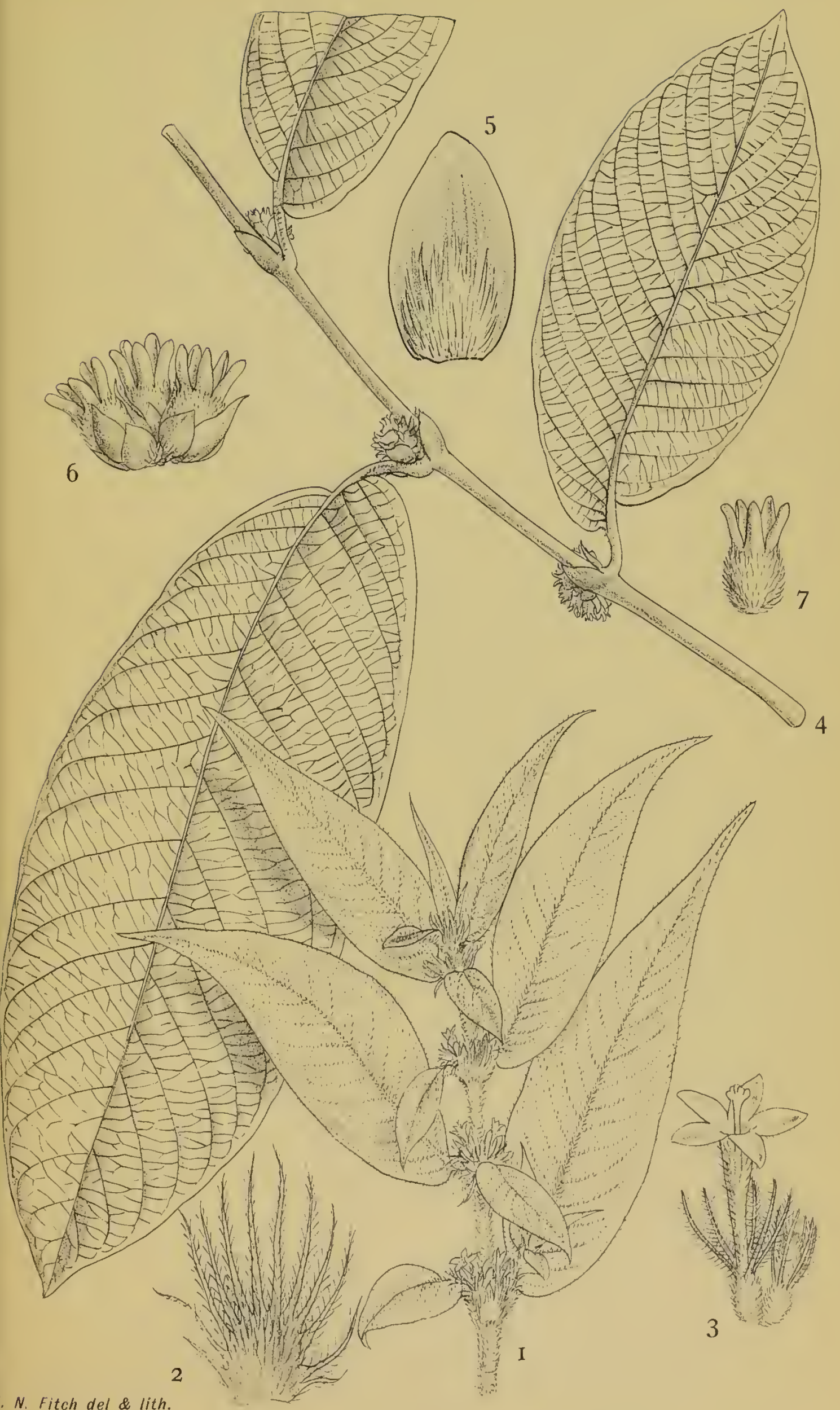




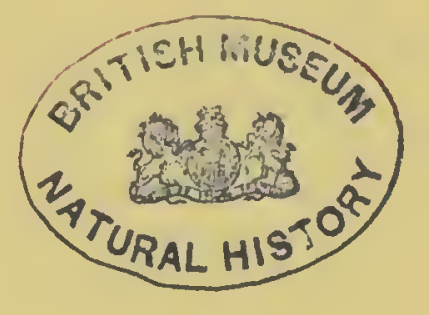




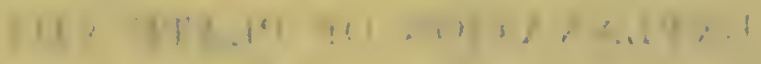

$$
\begin{aligned}
& \text {-2. . I num:s a } \\
& \text { an }+4,+\cdots, \ldots, 1 \\
& \text {, n. }
\end{aligned}
$$

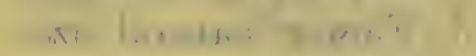

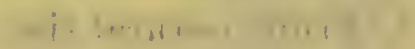

s. + il $:-\ldots, 11, m<$

$$
\begin{aligned}
& \text {., 1, ., , , , }
\end{aligned}
$$

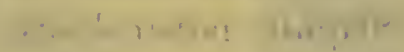

$$
\text { mit. 1. . . . mi, }
$$

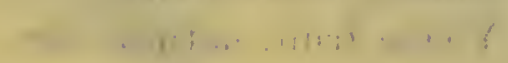




\section{EXPLANATION OF PLATE VTII}

S. uracilis Wernhem

1. Portion of shoot, $\times$ !.

$\therefore$. Stipule, natural size.

3. Hlower, natural size.

4. Fruit, natural size.

S. mattogrossensis Wernherm

5. Portion of shont, $\times \frac{1}{2}$.

6. Stipule, natural size.

7. H'lower, natural size.

's. Yomug frout, natural size. 


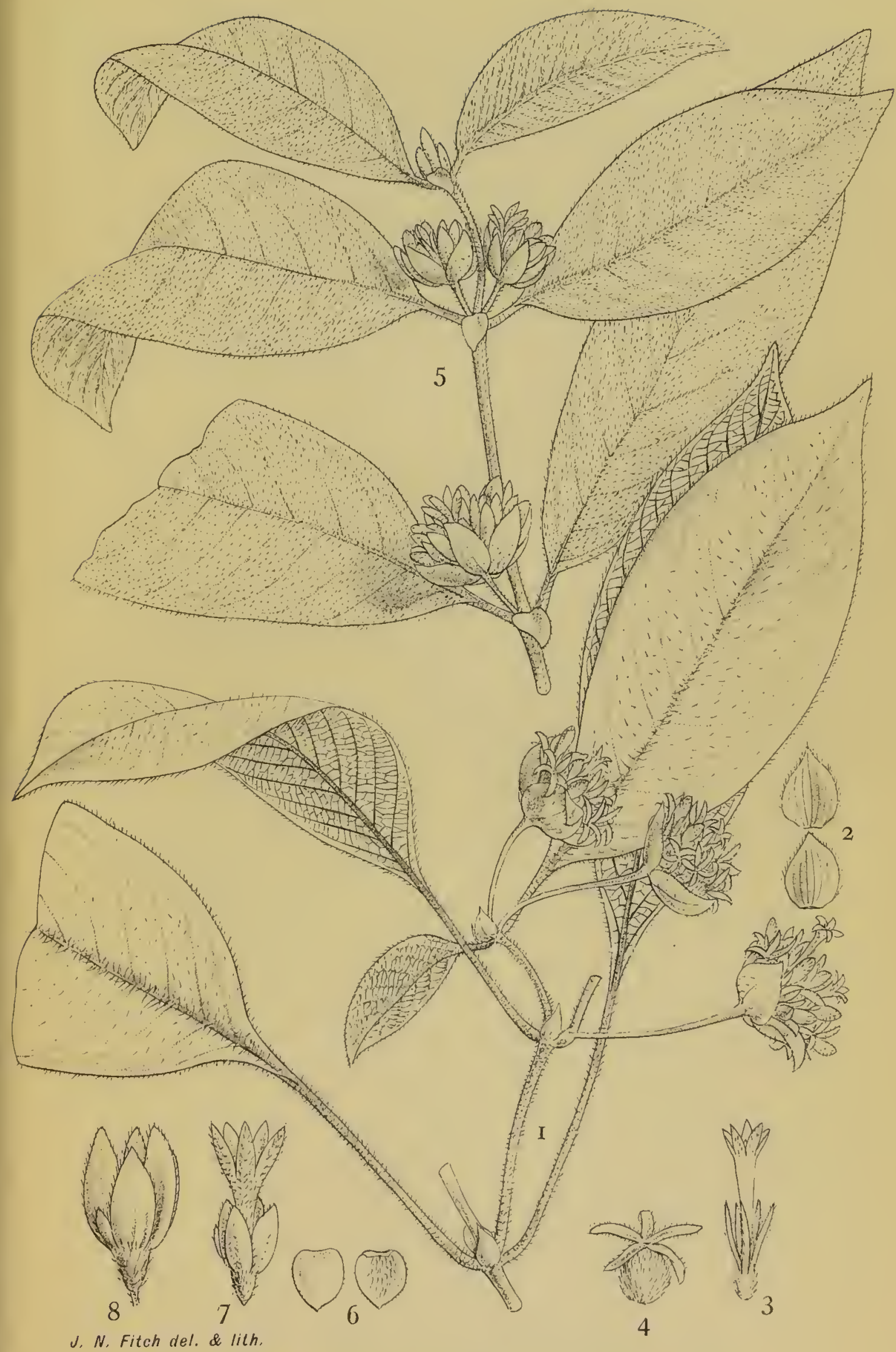

I-4, S. gracilis . 5-8, S. mattogrossensis. 


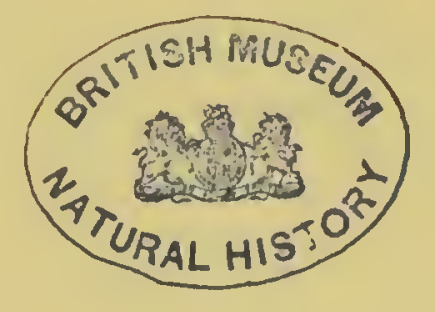



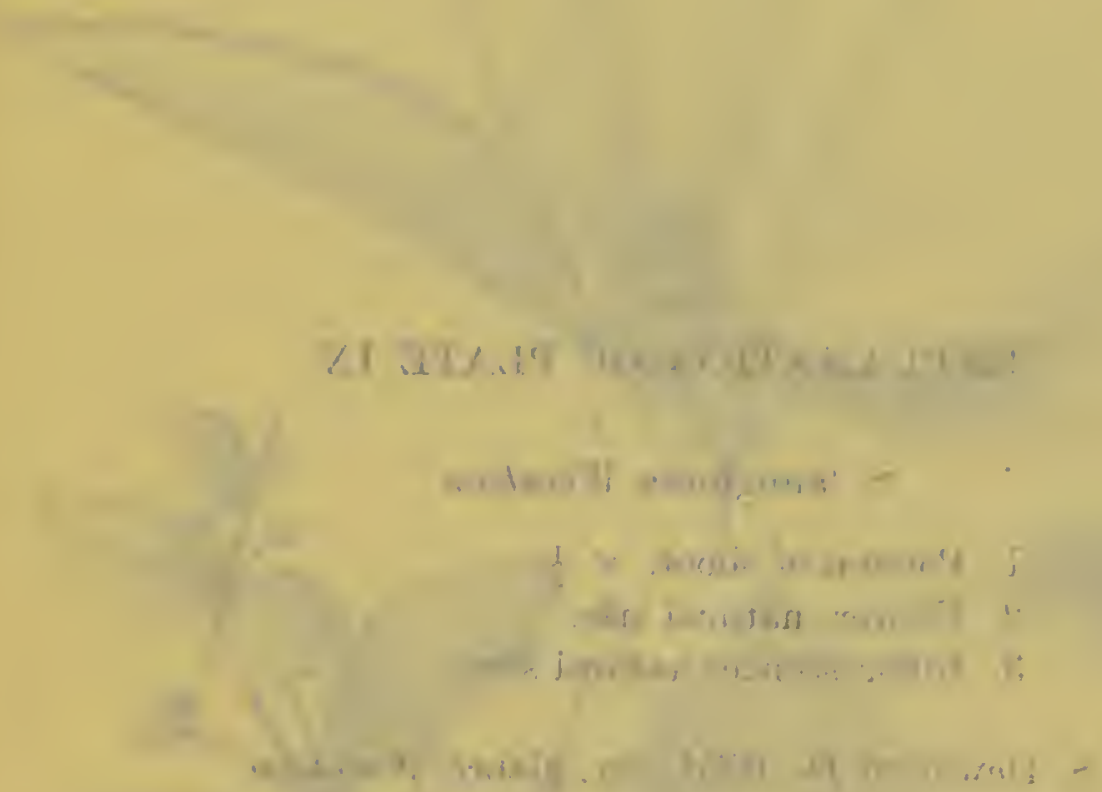

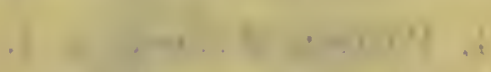

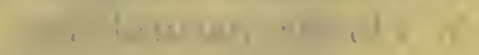
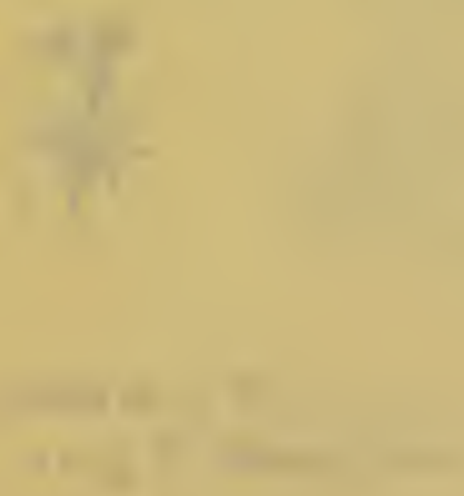


\title{
EXPLANATION OF PLATE IX
}

\author{
S. lanuginosa Wernham
}

1. Portion of shoot, $\times \frac{1}{2}$.

2. Flower, natural size.

3. Inflorescence, natural size.

S. Dewevrei De Wild. var. glabra Wernham

4. Portion of shoot, $\times \frac{1}{2}$.

๖. Flower, natural size. 


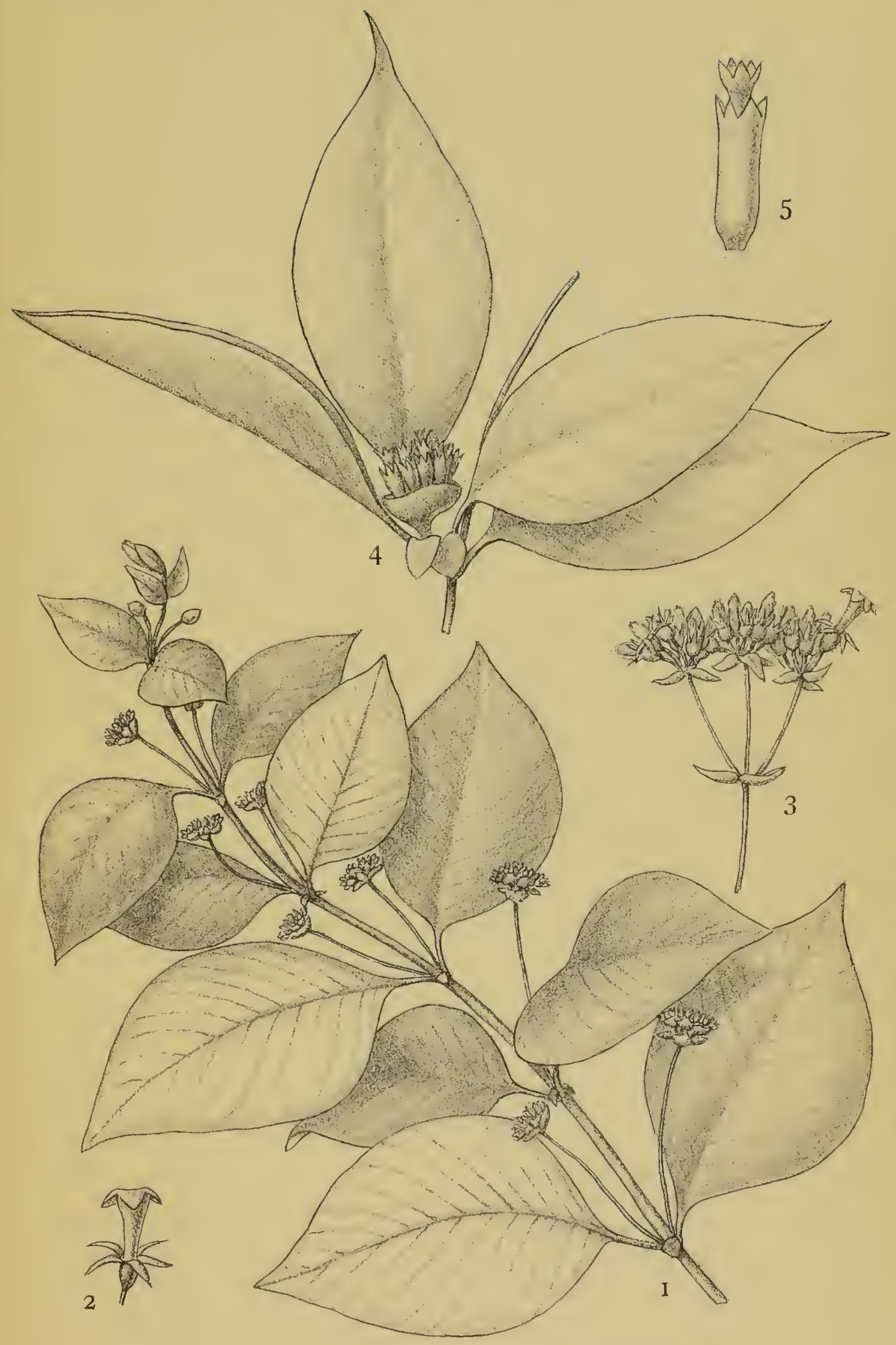

P. Highley del. \& lith.

I-3, S. lanuginosa. 4, 5, S. Dewevrei var. glabra. 


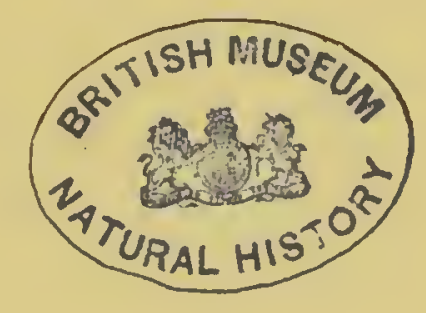




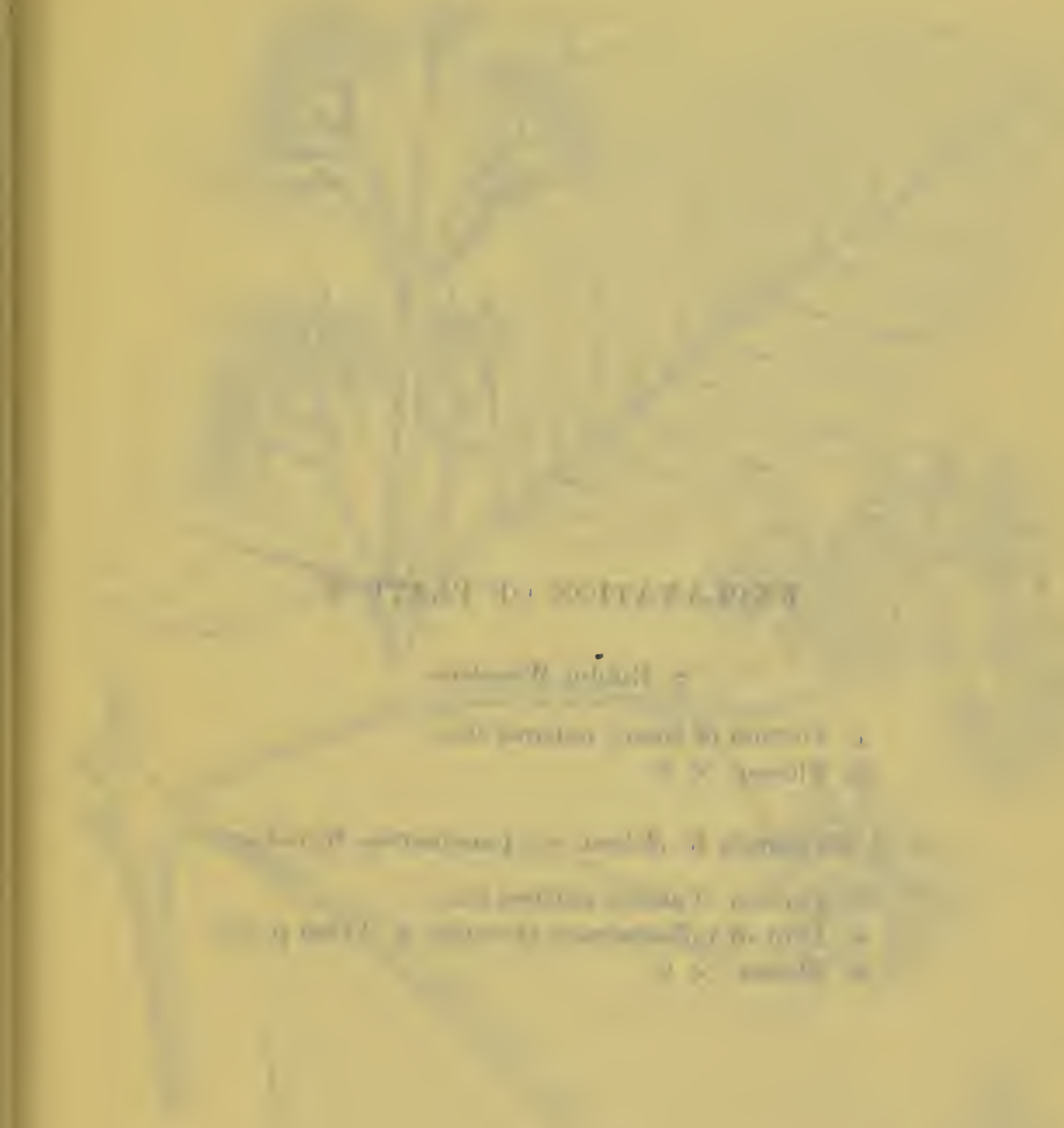


EXPLANATION OF PLATE X

\section{S. Robbii Wernham}

1. Portion of shoot, natural size.

2. Flower, $\times 2$.

S. floribunda $K$. Schum. var paucinervis Wernham

3. Portion of shoot, natural size.

4. Unit of inflorescence (young), $\times 2$ (see p. 15).

5. Elower, $\times 2$. 


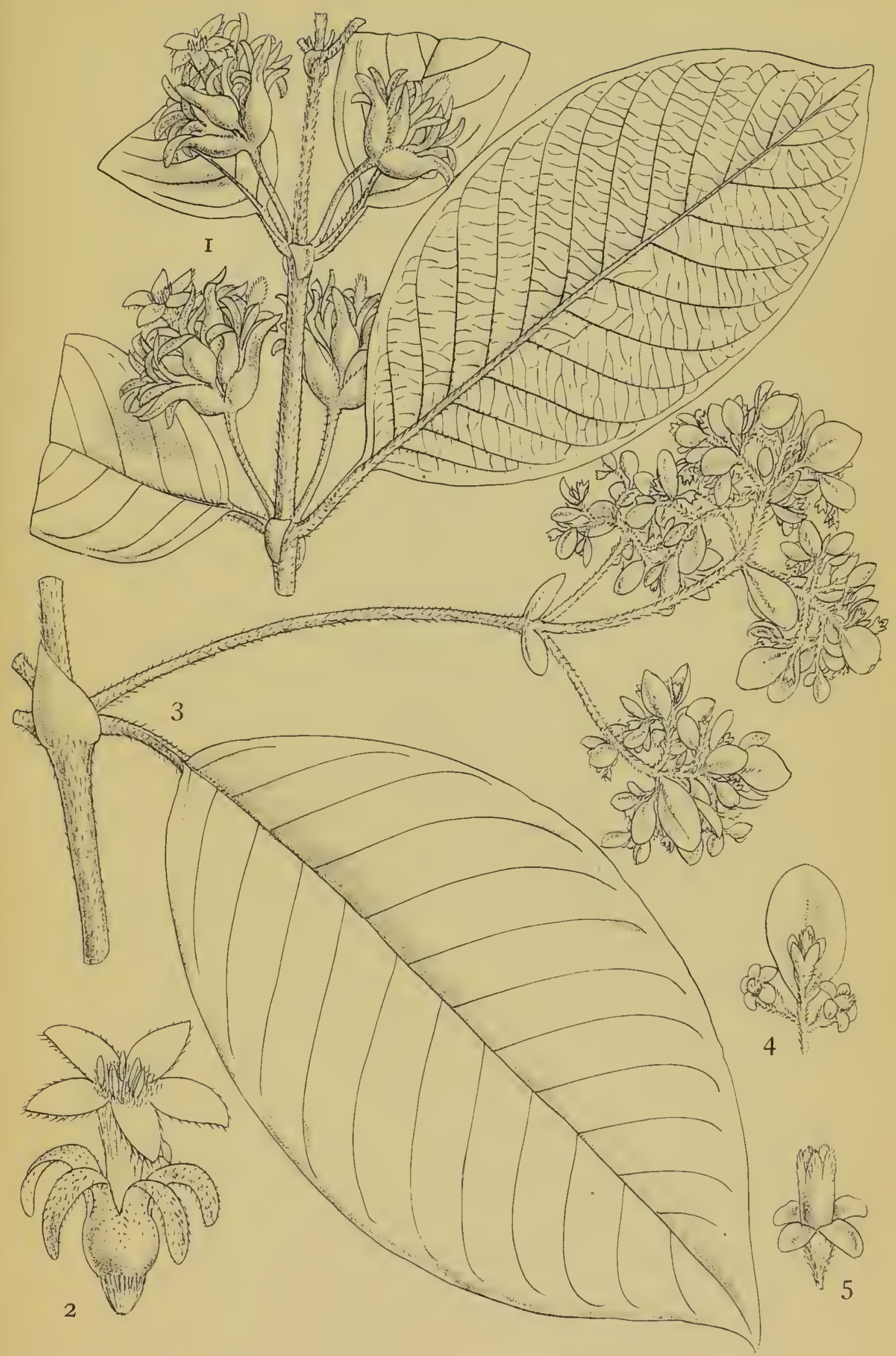

J. N. Fitch del. \& lith.

I, 2, S. Robbii. 3-5, S. floribunda var. paucinervis. 


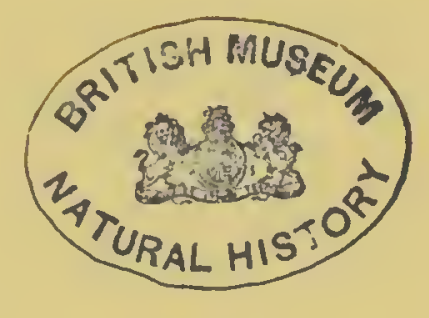




$$
\begin{aligned}
& \text {, }
\end{aligned}
$$

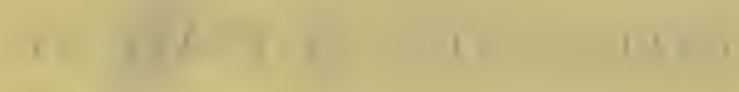

$$
\begin{aligned}
& \text { 1) }
\end{aligned}
$$

10.

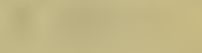

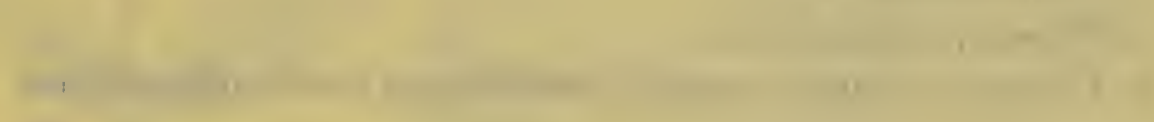
1

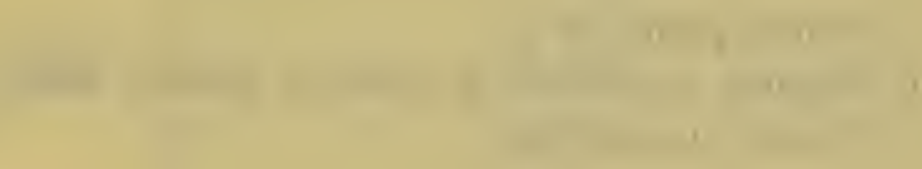




\title{
EXPLANATION OF PLATE XI
}

\author{
S. xanthotricha Wernham
}

1. Portion of young shoot, showing stipules and young leaf, $\times \frac{1}{3}$.

2. Mature leaf, $\times \frac{1}{3}$.

3. Flower, natural size.

4. Cluster of fruits, showing cauliflorous habit, natural size.

\section{S. Barteri Trernham}

5. Whole plant, $x \frac{1}{3}$.

6. Stipule, $a$, interior, $b$, exterior, natural size.

7. Flower, natural size. 


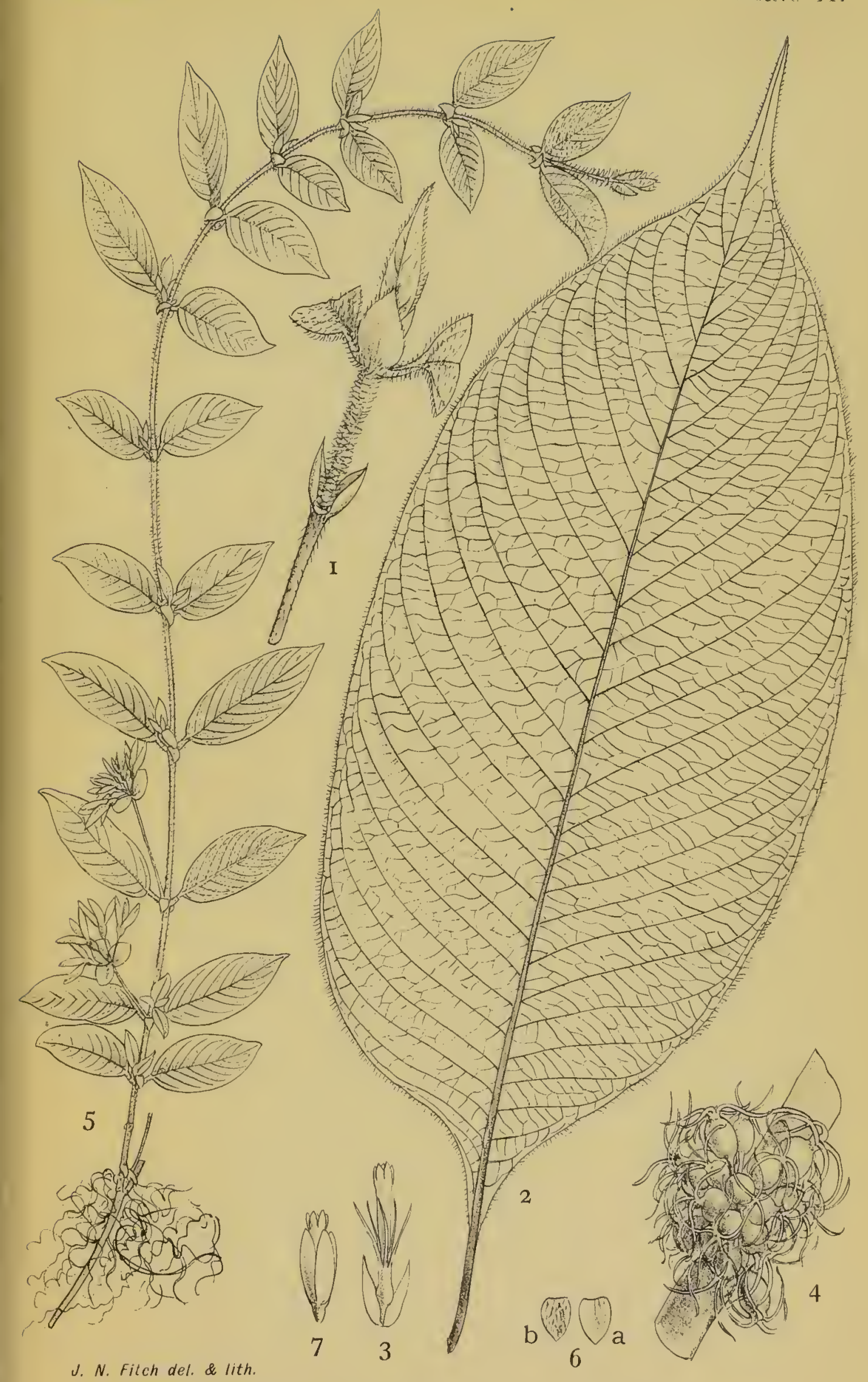

I-4, S. xanthotricha . 5-7, S. Barteri. 


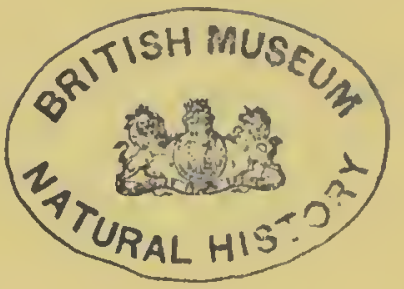




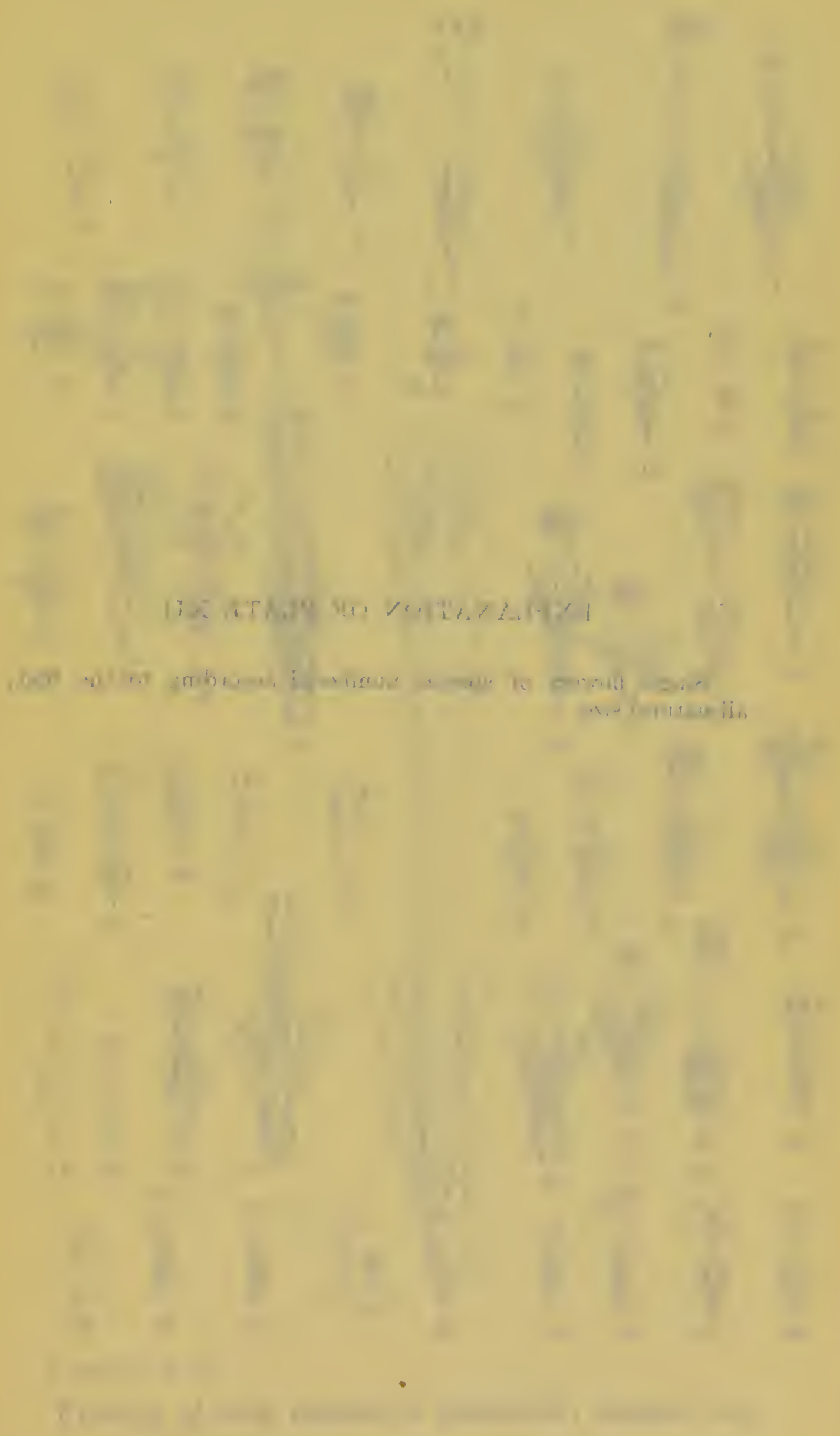


EXPLANATION OF PLATE XII

Single flowers of species numbered according to the text, all natural size. 


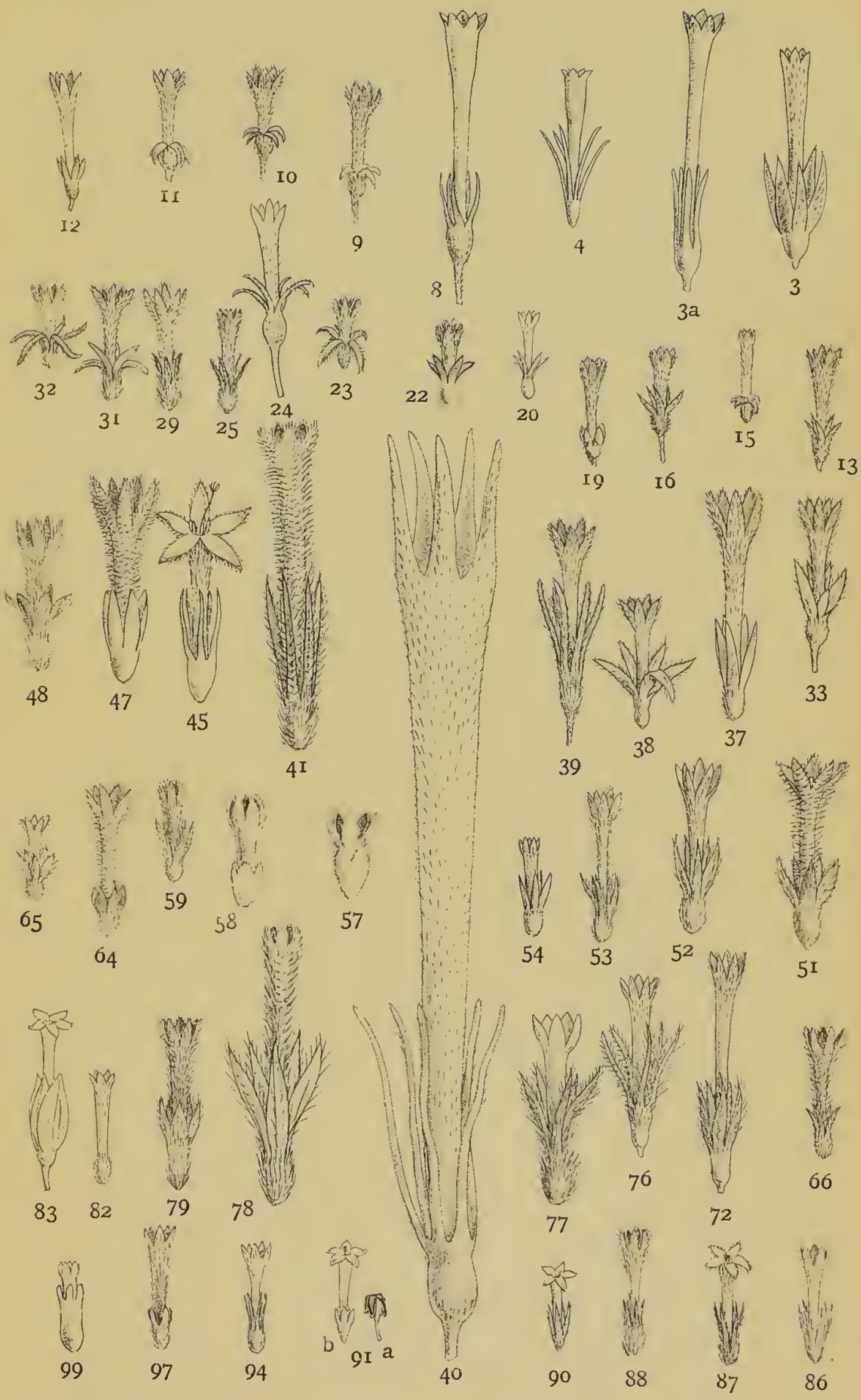

J. N. Fitch del. \& lith.

Flowers of each species as numbered; natural size. 





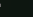


